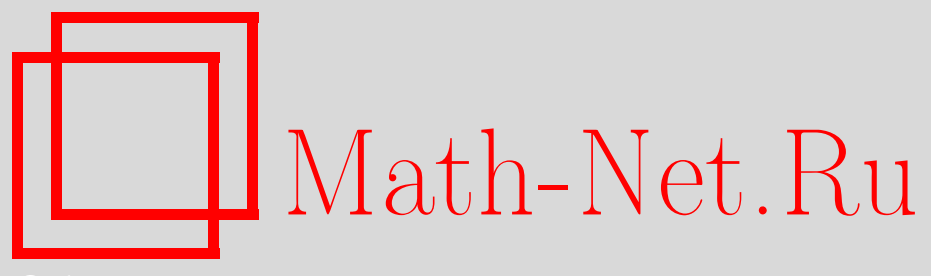

В. П. Спиридонов, Очерки теории эллиптических гипергеометрических функций, УМН, 2008, том 63, выпуск $3,3-72$

DOI: https://doi.org/10.4213/rm9197

Использование Общероссийского математического портала Math-Net.Ru подразумевает, что вы прочитали и согласны с пользовательским соглашением http://www . mathnet.ru/rus/agreement

Параметры загрузки:

IP : 54.164 .48 .24

26 апреля 2023 г., 15:41:30

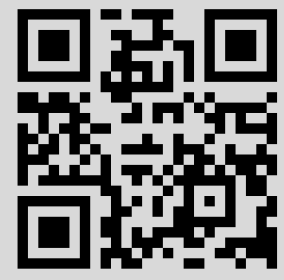


УДК $517.5+517.3$

\section{Очерки теории эллиптических гипергеометрических функций}

\section{В. П. Спиридонов}

Дается краткий обзор основных результатов теории эллиптических гипергеометрических функций - нового класса специальных функций математической физики. Доказывается самая общая известная точная формула однократного интегрирования, обобщающая бета-интеграл Эйлера, названная эллиптическим бета-интегралом. Строится эллиптический аналог гипергеометрической функции Гаусса и эллиптическое гипергеометрическое уравнение для него. Приводятся соотношения биортогональности для этой функции и ее частных случаев. Перечисляются известные эллиптические бета-интегралы на корневых системах и рассматриваются преобразования симметрии для соответствующих эллиптических гипергеометрических функций более высокого порядка.

Библиография: 118 названий.

\section{СоДЕРЖАНИЕ}

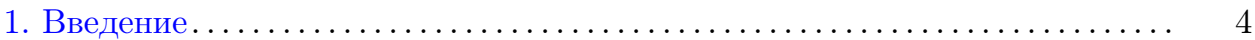

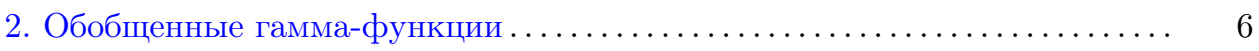

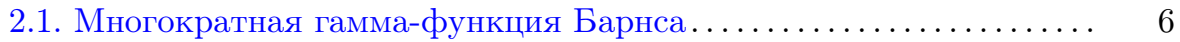

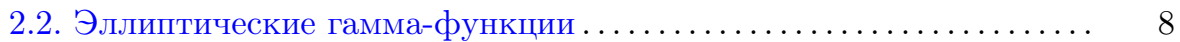

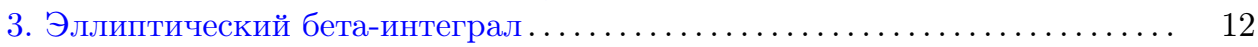

4. Общие эллиптические гипергеометрические ряды и интегралы ...... 18

4.1. Эллиптический аналог функции Мейера .................. 18

4.2. Вполне уравновешенные и совершенно уравновешенные интег-

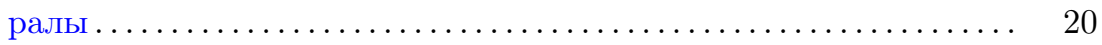

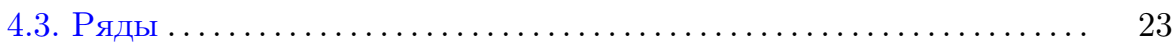

5. Эллиптический аналог гипергеометрической функции Гаусса ........ 26

5.1. Определение $V$-функции и связь с системой корней $E_{7} \ldots \ldots \ldots 26$

5.2. Эллиптическое гипергеометрическое уравнение ............ 28

6. Цепочки преобразований симметрии для функций ............... 31

7. Биортогональные функции гипергеометрического типа ........... 34

7.1. Дискретные биортогональные функции с непрерывной мерой... 34

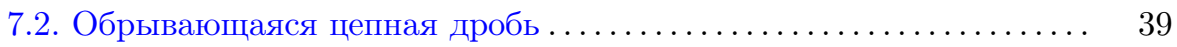

7.3. Непрерывная биортогональность $V$-функции .............. 41

8. Связь с алгеброй Склянина ............................... 43

(C) В. П. Спиридонов, 2008 
9. Разложения на простые дроби и определители $\ldots \ldots \ldots \ldots \ldots \ldots \ldots \ldots . \quad 46$

10. Эллиптические бета-интегралы на корневых системах ............ 48

10.1. Интегралы для системы корней $C_{n} \ldots \ldots \ldots \ldots \ldots \ldots \ldots \ldots, 48$

10.2. Интегралы для системы корней $A_{n} \ldots \ldots \ldots \ldots \ldots \ldots \ldots \ldots \ldots, 51$

11. Некоторые формулы суммирования многократных рядов ......... 54

12. Преобразования симметрии для многократных интегралов ........ 56

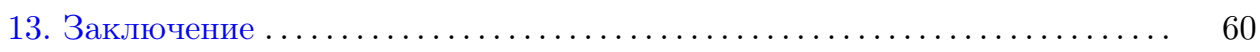

Приложение А. Эллиптические функции и тэта-функции Якоби . . . . . . 62

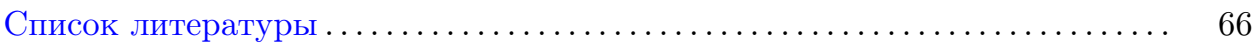

\section{1. Введение}

Теория специальных функций широко востребована в теоретической и математической физике как справочный набор точных математических формул и методов их построения. Это относится к формулам суммирования рядов, точно вычисляемым интегралам, преобразованиям симметрии функций, дифференциальным или другим уравнениям, решаемым в терминах “простых" функций, и так далее. Бурное построение такой базы данных, которое происходило в XIX веке и находилось на вершине приоритетов математики того времени, сменилось в XX веке значительным снижением интереса к специальным функциям, изучение которых стало рассматриваться как дело второстепенной важности. Такое отношение к исследованиям в этой области оправдывалось мнением, что все основные типы интересных функций "с классическими свойствами" (эллиптические, гипергеометрические, автоморфные и некоторые другие функции) уже найдены и необходимо только их более детальное изучение.

Теория специальных функций гипергеометрического типа развивалась в течение нескольких столетий, начиная с фундаментальных результатов, полученных Эйлером [1]. Над ее основаниями работали Гаусс, Якоби, Риман, Куммер и другие выдающиеся математики. Гипергеометрическая функция Гаусса ${ }_{2} F_{1}(a, b ; c ; x)$ является каноническим примером функций такого типа. Согласно подходу Похгаммера и Хорна [2], обобщенные чисто гипергеометрические ряды можно определить как суммы $\sum_{n} c_{n}$, для которых отношение $c_{n+1} / c_{n}$ есть рациональная функция $n$. В 1847 г. Гейне ввел в рассмотрение $q$-аналог ${ }_{2} F_{1}$-ряда ${ }_{2} \varphi_{1}(a, b ; c ; q ; x)[3]$. Общие ряды такого типа $\sum_{n} c_{n}$ характеризуются тем, что для них отношение $c_{n+1} / c_{n}$ есть рациональная функция $q^{n}$, где $q-$ некоторый комплексный параметр. До недавнего времени были известны только эти два класса функций гипергеометрического типа (включая интегральные представления для них), и их изучению было посвящено множество работ.

Около десяти лет назад неожиданно выяснилось, что существуют гипергеометрические функции третьего типа, связанные с эллиптическими кривыми. Впервые такие объекты возникли в рамках квантового метода обратной задачи рассеяния, разработанного для точно решаемых моделей статистической 
механики [4], [5], в качестве эллиптических решений уравнения Янга-Бакстера [6], [7]. Как продемонстрировано Френкелем и Тураевым [8], эти решения (названные эллиптическими $6 j$-символами) выражаются через эллиптическое обобщение обрывающегося совершенно уравновешенного сбалансированного $q$-гипергеометрического ряда $10 \varphi_{9}$ с дискретными значениями параметров. В работе [9] построена обобщенная $(1+1)$-мерная интегрируемая цепочка типа цепочки Тоды с дискретным временем и показано, что этот же обрывающийся ряд с произвольными параметрами возникает как частное решение соответствующих уравнений пары Лакса.

Общее формальное определение эллиптических гипергеометрических рядов, предложенное и детально изученное в [10], описывает их как ряды $\sum_{n} c_{n}$, для которых отношение $c_{n+1} / c_{n}$ является эллиптической функцией $n$. В рамках этой схемы случай [8] характеризуется наличием нескольких интересных структурных ограничений на коэффициенты $c_{n}$. При этом в описании бесконечных рядов имеются определенные трудности, связанные с их сходимостью. Поэтому общие эллиптические гипергеометрические функции определяются интегральными представлениями [11].

Наиболее общей чисто гипергеометрической функцией можно считать функцию Мейера [12]. Она задается контурным интегралом некоторого отношения гамма-функций Эйлера. Для интегральных представлений более сложных функций гипергеометрического типа требуются обобщенные гамма-функции, теория которых была развита Барнсом [13] и Джексоном [14] более века назад. $q$-гамма-функция Джексона необходима для описания $q$-гипергеометрических функций при $|q|<1$. Более сложная функция требуется, когда $q$ лежит на единичной окружности, $|q|=1$ [15]-[18]. Эти функции, связанные с гамма-функцией Барнса второго порядка, активно используются в современной математической физике в описании квантовых интегрируемых моделей и представлений квантовых алгебр [19]-[24].

Для определения эллиптических гипергеометрических интегралов требуется эллиптическая гамма-функция, связанная с гамма-функцией Барнса третьего порядка. Важность эллиптической гамма-функции была подчеркнута Руджинарсом [18], который дал ей это имя и рассмотрел некоторые ее свойства. Модулярные преобразования этой функции описаны в [25]. В [11] построена модифицированная эллиптическая гамма-функция, которая остается корректно определенной и в случае, когда один из базисных параметров лежит на единичной окружности. Другие аспекты этой функции изучались в [26]-[28].

Первая точная формула интегрирования, использующая эллиптическую гамма-функцию, была построена автором в [29]. Она представляет наиболее общий известный однократный интеграл, обобщающий бета-интеграл Эйлеpa [1]. Этот эллиптический бета-интеграл служит основой для построения общих совершенно уравновешенных эллиптических гипергеометрических функций. Первое направление обобщения состоит в увеличении числа свободных параметров, входящих в подынтегральное выражение, что приводит к эллиптическим аналогам функций ${ }_{s+1} F_{s}$ [11], [30]. В частности, таким способом строится эллиптический аналог гипергеометрической функции Гаусса и выводятся его свойства [31]. Второе направление обобщения увеличивает кратно- 
сти интегрирований так, что подынтегральные функции приобретают симметрии по переменным интегрирования, связанные с корневыми системами [11], [32]-[36]. Одно из таких обобщений приводит к эллиптическому аналогу интеграла Сельберга. За короткий период времени в работах, упомянутых выше и перечисленных ниже в списке литературы, была построена систематическая теория эллиптических гипергеометрических функций одной и многих переменных. Краткому описанию этой теории и посвящен настоящий обзор.

\section{2. Обобщенные гамма-функции}

Используемые символы: $\mathbb{Z}=0, \pm 1, \pm 2, \ldots ; \mathbb{C}$ - открытая комплексная плоскость; $\mathbb{C}^{*}=\mathbb{C} /\{0\} ; \mathbb{R}-$ действительная ось $; i=\sqrt{-1}$.

2.1. Многократная гамма-функция Барнса. Гамма-функция Эйлера является краеугольным камнем теории обычных гипергеометрических функций [1]. Различные аналоги этой функции со многими параметрами рассматривались в математической литературе начала двадцатого века. Наиболее полные исследования обобщенных гамма-функций принадлежат Барнсу [13]. Отправной точкой в его работе служит функция, обобщающая дзета-функцию Гурвица [1]

$$
\zeta(s, u)=\sum_{n=0}^{\infty} \frac{1}{(u+n)^{s}}, \quad \operatorname{Re}(s)>1,
$$

которая сводится к дзета-функции Римана при $u=1$. Ввиду возросшего интереса теория Барнса достаточно широко обсуждалась недавно в литературе, например в [19], [26]-[28], [37].

Рассмотрим $m$ квазипериодов $\omega_{j} \in \mathbb{C}$, которые для простоты будем считать линейно независимыми над $\mathbb{Z}$ (условие несоизмеримости). Для $s, u \in \mathbb{C}$ дзета-функция Барнса определяется $m$-кратным рядом

$$
\zeta_{m}(s, u ; \boldsymbol{\omega})=\sum_{n_{1}, \ldots, n_{m}=0}^{\infty} \frac{1}{(u+\Omega)^{s}}, \quad \Omega=n_{1} \omega_{1}+\cdots+n_{m} \omega_{m}
$$

сходящимся при $\operatorname{Re}(s)>m$ и при условии, что все $\omega_{j}$ лежат в одной полуплоскости, определяемой какой-либо прямой, проходящей через начало координат. Благодаря последнему требованию, последовательности $n_{1} \omega_{1}+\cdots+n_{m} \omega_{m}$ не имеют точек накопления в конечной плоскости при каких-либо $n_{j} \rightarrow+\infty$. Удобно считать, для определенности, что $\operatorname{Re}\left(\omega_{j}\right)>0$ или $\operatorname{Im}\left(\omega_{j}\right)>0$.

Функция $\zeta_{m}(s, u ; \boldsymbol{\omega})$ удовлетворяет следующему набору конечно-разностных уравнений:

$$
\zeta_{m}\left(s, u+\omega_{j} ; \boldsymbol{\omega}\right)-\zeta_{m}(s, u ; \boldsymbol{\omega})=-\zeta_{m-1}(s, u ; \boldsymbol{\omega}(j)), \quad j=1, \ldots, m,
$$

где $\boldsymbol{\omega}(j)=\left(\omega_{1}, \ldots, \omega_{j-1}, \omega_{j+1}, \ldots, \omega_{m}\right)$ и $\zeta_{0}(s, u ; \boldsymbol{\omega})=u^{-s}$. Она может быть аналитически (мероморфно) продолжена на всю комплексную плоскость $\operatorname{Re}(s) \leqslant m$ с простыми полюсами в точках $s=1,2, \ldots, m$. Многократная гамма-функция Барнса определятся равенством

$$
\Gamma_{m}(u ; \boldsymbol{\omega})=\left.\exp \left(\frac{\partial \zeta_{m}(s, u ; \boldsymbol{\omega})}{\partial s}\right)\right|_{s=0} .
$$


Она имеет представление в виде бесконечного произведения

$$
\frac{1}{\Gamma_{m}(u ; \boldsymbol{\omega})}=e^{\sum_{k=0}^{m} \gamma_{m k} \frac{u^{k}}{k !}} u \prod_{n_{1}, \ldots, n_{m}=0}^{\infty}\left(1+\frac{u}{\Omega}\right) e^{\sum_{k=1}^{m}(-1)^{k} \frac{u^{k}}{k \Omega^{k}}},
$$

где $\gamma_{m k}$ - некоторые постоянные, аналогичные постоянной Эйлера. Штрих произведения означает, что точка $n_{1}=\cdots=n_{m}=0$ в нем пропущена. В частности, функция $\Gamma_{1}(u ; \omega)$ прямым образом связана с гамма-функцией Эйлера $\Gamma(u)$ :

$$
\Gamma_{1}(u ; \omega)=\frac{\omega^{u / \omega}}{\sqrt{2 \pi \omega}} \Gamma\left(\frac{u}{\omega}\right)
$$

в чем можно убедиться непосредственными манипуляциями с дзета-функций Гурвица. Отметим, что в [13] Барнс использовал другую нормировку $\Gamma_{m}$-функции, в которой $\gamma_{m 0}=0$.

Функция $\Gamma_{m}(u ; \boldsymbol{\omega})$ удовлетворяет $m$ конечно-разностным уравнениям первого порядка, получающимся дифференцированием равенств (2.1) в точке $s=0$ :

$$
\Gamma_{m}\left(u+\omega_{j} ; \boldsymbol{\omega}\right)=\frac{1}{\Gamma_{m-1}(u ; \boldsymbol{\omega}(j))} \Gamma_{m}(u ; \boldsymbol{\omega}), \quad j=1, \ldots, m,
$$

где $\Gamma_{0}(u ; \boldsymbol{\omega}):=u^{-1}$.

Хорошо известны интегральные представления для гамма-функции Эйлера:

$$
\Gamma(s)=\int_{0}^{\infty} t^{s-1} e^{-t} d t=\frac{i}{2 \sin (\pi s)} \int_{C_{H}}(-t)^{s-1} e^{-t} d t,
$$

где в первом случае $\operatorname{Re}(s)>0$, а во втором выражении $|\arg (-t)|<\pi$ и ганкелевский контур $C_{H}$ начинается и заканчивается вблизи точки $+\infty$, огибая полуось $[0, \infty)$ против часовой стрелки. С их помощью можно записать

$$
\begin{aligned}
\zeta_{m}(s, u ; \boldsymbol{\omega}) & =\frac{1}{\Gamma(s)} \int_{0}^{\infty} \frac{t^{s-1} e^{-u t}}{\prod_{k=1}^{m}\left(1-e^{-\omega_{k} t}\right)} d t \\
& =\frac{i \Gamma(1-s)}{2 \pi} \int_{C_{H}} \frac{(-t)^{s-1} e^{-u t}}{\prod_{k=1}^{m}\left(1-e^{-\omega_{k} t}\right)} d t
\end{aligned}
$$

и аналитически продолжить эту функцию по $s$ на всю комплексную плоскость. Используя последнее выражение, Барнс получил для многократных гамма-функций следующее интегральное представление:

$$
\Gamma(u ; \boldsymbol{\omega})=\exp \left(\frac{1}{2 \pi i} \int_{C_{H}} \frac{e^{-u t}(\log (-t)+\gamma)}{t \prod_{k=1}^{m}\left(1-e^{-\omega_{k} t}\right)} d t\right),
$$

где $\gamma-$ постоянная Эйлера.

Значения $\zeta_{m}(0, u ; \boldsymbol{\omega})$ выражаются через многократные многочлены Бернулли,

$$
\zeta_{m}(0, u ; \boldsymbol{\omega})=\frac{(-1)^{m}}{m !} B_{m, m}(u \mid \boldsymbol{\omega}),
$$

которые определяются производящей функцией

$$
\frac{x^{m} e^{x u}}{\prod_{k=1}^{m}\left(e^{\omega_{k} x}-1\right)}=\sum_{n=0}^{\infty} B_{m, n}\left(u \mid \omega_{1}, \ldots, \omega_{m}\right) \frac{x^{n}}{n !} .
$$


Нам потребуются в дальнейшем первые три диагональных многочлена

$$
\begin{aligned}
B_{1,1}\left(u \mid \omega_{1}\right) & =\frac{u}{\omega_{1}}-\frac{1}{2}, \\
B_{2,2}\left(u \mid \omega_{1}, \omega_{2}\right) & =\frac{1}{\omega_{1} \omega_{2}}\left(u^{2}-\left(\omega_{1}+\omega_{2}\right) u+\frac{\omega_{1}^{2}+\omega_{2}^{2}}{6}+\frac{\omega_{1} \omega_{2}}{2}\right), \\
B_{3,3}\left(u \mid \omega_{1}, \omega_{2}, \omega_{3}\right) & =\frac{1}{\omega_{1} \omega_{2} \omega_{3}}\left(u^{3}-\frac{3 u^{2}}{2} \sum_{k=1}^{3} \omega_{k}+\frac{u}{2}\left(\sum_{k=1}^{3} \omega_{k}^{2}+3 \sum_{j<k} \omega_{j} \omega_{k}\right)\right. \\
& \left.-\frac{1}{4}\left(\sum_{k=1}^{3} \omega_{k}\right) \sum_{j<k} \omega_{j} \omega_{k}\right) .
\end{aligned}
$$

Теория обычных гипергеометрических функций строится с помощью гаммафункции Эйлера или $\Gamma_{1}\left(u ; \omega_{1}\right) ; q$-гипергеометрические функции привязаны к $\Gamma_{2}\left(u ; \omega_{1}, \omega_{2}\right)$, а эллиптические гипергеометрические функции “живут" на уровне многократной гамма-функции Барнса третьего порядка соответственно.

2.2. Эллиптические гамма-функции. Пусть $\omega_{1}, \omega_{2}, \omega_{3}$ обозначают комплексные параметры, линейно независимые над $\mathbb{Z}$ и лежащие в правой полуплоскости. Определим с их помощью базисные переменные $p, q, r \in \mathbb{C}$ :

$$
q=e^{2 \pi i \omega_{1} / \omega_{2}}, \quad p=e^{2 \pi i \omega_{3} / \omega_{2}}, \quad r=e^{2 \pi i \omega_{3} / \omega_{1}},
$$

и их модулярно $(\tau \rightarrow-1 / \tau)$ преобразованных партнеров:

$$
\tilde{q}=e^{-2 \pi i \omega_{2} / \omega_{1}}, \quad \tilde{p}=e^{-2 \pi i \omega_{2} / \omega_{3}}, \quad \tilde{r}=e^{-2 \pi i \omega_{1} / \omega_{3}} .
$$

При $|p|,|q|<1$ бесконечные произведения

$$
(z ; q)_{\infty}=\prod_{j=0}^{\infty}\left(1-z q^{j}\right), \quad(z ; p, q)_{\infty}=\prod_{j, k=0}^{\infty}\left(1-z p^{j} q^{k}\right)
$$

определены и удовлетворяют $q$-разностным уравнениям

$$
\begin{gathered}
(q z ; q)_{\infty}=\frac{(z ; q)_{\infty}}{1-z} \\
(q z ; q, p)_{\infty}=\frac{(z ; q, p)_{\infty}}{(z ; p)_{\infty}}, \quad(p z ; q, p)_{\infty}=\frac{(z ; q, p)_{\infty}}{(z ; q)_{\infty}}
\end{gathered}
$$

Укороченная тэта-функция

$$
\theta(z ; p):=(z ; p)_{\infty}\left(p z^{-1} ; p\right)_{\infty}=\frac{1}{(p ; p)_{\infty}} \sum_{k \in \mathbb{Z}}(-1)^{k} p^{k(k-1) / 2} z^{k}
$$

играет ключевую роль в наших рассмотрениях. Она обладает следующими простыми преобразованиями симметрии:

$$
\theta(p z ; p)=\theta\left(z^{-1} ; p\right)=-z^{-1} \theta(z ; p)
$$


и имеет нули, $\theta(z ; p)=0$, при $z=p^{k}, k \in \mathbb{Z}$. Очевидно, что $\theta(z ; 0)=1-z$. Для $k>0$ имеем

$$
\theta\left(p^{k} z ; p\right)=\frac{\theta(z ; p)}{(-z)^{k} p^{\left(\begin{array}{c}
k \\
2
\end{array}\right)}}, \quad \theta\left(p^{-k} z ; p\right)=\frac{(-z)^{k} \theta(z ; p)}{p^{\left(\begin{array}{c}
k+1 \\
2
\end{array}\right)}} .
$$

Нам потребуется правило $\tau \rightarrow-1 / \tau$ модулярного преобразования $\theta(z ; p)$, для описания которого необходимо воспользоваться экспоненциальной параметризацией переменных:

$$
\theta\left(e^{-2 \pi i u / \omega_{1}} ; e^{-2 \pi i \omega_{2} / \omega_{1}}\right)=e^{\pi i B_{2,2}\left(u \mid \omega_{1}, \omega_{2}\right)} \theta\left(e^{2 \pi i u / \omega_{2}} ; e^{2 \pi i \omega_{1} / \omega_{2}}\right),
$$

где $B_{2,2}\left(u \mid \omega_{1}, \omega_{2}\right)$ - второй многочлен Бернулли. В дальнейшем удобно использовать компактные обозначения

$$
\theta\left(a_{1}, \ldots, a_{k} ; p\right):=\theta\left(a_{1} ; p\right) \cdots \theta\left(a_{k} ; p\right), \quad \theta\left(a t^{ \pm 1} ; p\right):=\theta(a t ; p) \theta\left(a t^{-1} ; p\right) .
$$

Простейшую гамма-функцию можно определить как специальное мероморфное решение функционального уравнения $f\left(u+\omega_{1}\right)=u f(u)$. Следуя подходу Джексона [14], мы будем связывать q-гамма-функции с мероморфными решениями уравнения

$$
f\left(u+\omega_{1}\right)=\left(1-e^{2 \pi i u / \omega_{2}}\right) f(u),
$$

где $q=e^{2 \pi i \omega_{1} / \omega_{2}}$. Вводя переменную $z=e^{2 \pi i u / \omega_{2}}$, это уравнение можно заменить на $f(q z)=(1-z) f(z)$. При $|q|<1$ его частное решение, аналитическое в точке $z=0$, находится простой итерацией, что дает стандартную $q$-гамма-функцию $\gamma_{q}(z)=1 /(z ; q)_{\infty}$ (которую можно рассматривать также как $q$-экспоненциальную функцию [3]). Это выражение отличается от $q$-гаммафункции Джексона

$$
\Gamma_{q}^{(J)}(u)=\frac{(q ; q)_{\infty}}{\left(q^{u} ; q\right)_{\infty}}(1-q)^{1-u}
$$

удовлетворяющей уравнению $\Gamma_{q}^{(J)}(u+1) / \Gamma_{q}^{(J)}(u)=\left(1-q^{u}\right) /(1-q)$, заменой аргумента и простым множителем. Предельный переход к обычной гамма-функции имеет вид $\lim _{q \rightarrow 1} \Gamma_{q}^{(J)}(u)=\Gamma(u)$ [1], [3], однако для упрощения $q$-гипергеометрических формул удобнее пользоваться функцией $\gamma_{q}(z)$.

Модифицированная $q$-гамма-функция, которая остается корректно определенной и при $|q|=1$, имеет вид

$$
\gamma\left(u ; \omega_{1}, \omega_{2}\right)=\exp \left(-\int_{\mathbb{R}+i 0} \frac{e^{u x}}{\left(1-e^{\omega_{1} x}\right)\left(1-e^{\omega_{2} x}\right)} \frac{d x}{x}\right),
$$

где контур $\mathbb{R}+i 0$ проходит по действительной оси, огибая сверху точку $x=0$ инфинитезимальным образом. Эта функция появлялась в теории чисел [15] и в теории интегрируемых систем [17]-[19]. Она фигурирует в литературе под различными именами: “двойной синус" [16], "некомпактный квантовый дилогарифм" [21], “гиперболическая гамма-функция" [18].

Пусть $\operatorname{Re}\left(\omega_{1}\right), \operatorname{Re}\left(\omega_{2}\right)>0$. Тогда интеграл (2.14) сходится при $0<\operatorname{Re}(u)<$ $\operatorname{Re}\left(\omega_{1}+\omega_{2}\right)$. При подходящих ограничениях на $u$ и $\omega_{1,2}$ интеграл $(2.14)$ может 
быть вычислен как сходящаяся сумма вычетов полюсов в верхней полуплоскости. При $\operatorname{Im}\left(\omega_{1} / \omega_{2}\right)>0$ это приводит к выражению

$$
\gamma\left(u ; \omega_{1}, \omega_{2}\right)=\frac{\left(e^{2 \pi i u / \omega_{1}} \tilde{q} ; \tilde{q}\right)_{\infty}}{\left(e^{2 \pi i u / \omega_{2}} ; q\right)_{\infty}}
$$

которое аналитически продолжается на всю комплексную плоскость $u$ (это выражение очевидным образом удовлетворяет уравнению (2.12)). Данная $q$-гамма-функция служит главным "кирпичиком" при построении аналитических $q$-гипергеометрических функций при $|q|=1$, которые не рассматривались в литературе до недавнего времени.

Модифицированная $q$-гамма-функция пропорциональна отношению двух гамма-функций Барнса второго порядка. Общее соотношение такого типа выводится с помощью интегрального представления (2.4) и имеет вид [27]

$$
\exp \left(-\int_{\mathbb{R}+i 0} \frac{e^{u x}}{\prod_{k=1}^{m}\left(e^{\omega_{k} x}-1\right)} \frac{d x}{x}\right)=e^{\frac{\pi i}{m !} B_{m, m}(u \mid \boldsymbol{\omega})} \frac{\Gamma_{m}(u ; \boldsymbol{\omega})^{(-1)^{m}}}{\Gamma_{m}\left(\sum_{k=1}^{m} \omega_{k}-u ; \boldsymbol{\omega}\right)},
$$

где $\operatorname{Re}\left(\omega_{k}\right)>0$ и $0<\operatorname{Re}(u)<\operatorname{Re}\left(\sum_{k=1}^{m} \omega_{k}\right)$. В [27] также были получены представления этих функций в виде бесконечных произведений, аналогичных (2.15), которые мы не приводим.

Еще в работах Барнса было отмечено, что $\theta_{1}(u \mid \tau)$-функция Якоби может быть разложена на произведение четырех многократных гамма-функций второго порядка с различными аргументами. Точная форма такого соотношения имеет вид (см., например, [26])

$$
\theta\left(e^{2 \pi i u} ; p\right)=\frac{e^{-\pi i B_{2,2}(u \mid 1, \tau)}}{\Gamma_{2}(u ; 1, \tau) \Gamma_{2}(1+\tau-u ; 1, \tau) \Gamma_{2}(u-\tau ; 1,-\tau) \Gamma_{2}(1-u ; 1,-\tau)},
$$

где $p=e^{2 \pi i \tau}$. Общая связь между многократными гамма-функциями и бесконечными произведениями джексоновского типа имеет вид [26]

$$
\prod_{n_{1}, \ldots, n_{m}=0}^{\infty}\left(1-e^{2 \pi i(u+\Omega)}\right)=\frac{e^{-\pi i \zeta_{m+1}(0, u ; 1, \alpha)}}{\Gamma_{m+1}(u ; 1, \alpha) \Gamma_{m+1}(1-u ; 1,-\alpha)},
$$

где $\alpha=\left(\alpha_{1}, \ldots, \alpha_{m}\right), \Omega=n_{1} \alpha_{1}+\cdots+n_{m} \alpha_{m}$ и $\operatorname{Im}\left(\alpha_{j}\right)>0$.

Следуя логике определения $q$-гамма-функций, мы связываем эллиптические гамма-функции с мероморфными решениями разностного уравнения

$$
f\left(u+\omega_{1}\right)=\theta\left(e^{2 \pi i u / \omega_{2}} ; p\right) f(u),
$$

переходящего в (2.12) при $p \rightarrow 0$. Пользуясь факторизацией (2.9) и равенствами (2.8), нетрудно убедиться, что отношение

$$
\Gamma(z ; p, q)=\frac{\left(p q z^{-1} ; p, q\right)_{\infty}}{(z ; p, q)_{\infty}}=\prod_{j, k=0}^{\infty} \frac{1-z^{-1} p^{j+1} q^{k+1}}{1-z p^{j} q^{k}}
$$

где $|p|,|q|<1$ и $z \in \mathbb{C}^{*}$, удовлетворяет уравнениям

$$
\Gamma(q z ; p, q)=\theta(z ; p) \Gamma(z ; p, q), \quad \Gamma(p z ; p, q)=\theta(z ; q) \Gamma(z ; p, q) .
$$


Таким образом, функция $f(u)=\Gamma\left(e^{2 \pi i u / \omega_{2}} ; p, q\right)$ определяет решение уравнения (2.17) при $|q|,|p|<1$ и называется (стандартной) эллиптической гаммафункцией [18]. Так как не существует нетривиальных трижды периодических функций, ее можно однозначно определить как мероморфное решение системы трех уравнений:

$$
f\left(u+\omega_{2}\right)=f(u), \quad f\left(u+\omega_{3}\right)=\theta\left(e^{2 \pi i u / \omega_{2}} ; q\right) f(u)
$$

и уравнения (2.17) с нормировкой решения $f\left(\sum_{k=1}^{3} \omega_{k} / 2\right)=1$. Формула отражения для этой обобщенной гамма-функции имеет вид $\Gamma(z ; p, q) \Gamma(p q / z ; p, q)=1$. При $p=0$ имеем $\Gamma(z ; 0, q)=\gamma_{q}(z)$.

Модифицированная эллиптическая гамма-функция, корректно определенная и при $|q|=1$, имеет вид [11]

$$
G(u ; \boldsymbol{\omega})=\Gamma\left(e^{2 \pi i u / \omega_{2}} ; p, q\right) \Gamma\left(r e^{-2 \pi i u / \omega_{1}} ; \tilde{q}, r\right) .
$$

Она определяет однозначное решение трех уравнений:

$$
f\left(u+\omega_{2}\right)=\theta\left(e^{2 \pi i u / \omega_{1}} ; r\right) f(u), \quad f\left(u+\omega_{3}\right)=e^{-\pi i B_{2,2}(u \mid \boldsymbol{\omega})} f(u)
$$

и уравнения (2.17) с нормировкой решения $f\left(\sum_{k=1}^{3} \omega_{k} / 2\right)=1$.

Нетрудно проверить [38], что функция

$$
G(u ; \boldsymbol{\omega})=e^{-\frac{\pi i}{3} B_{3,3}(u \mid \boldsymbol{\omega})} \Gamma\left(e^{-2 \pi i u / \omega_{3}} ; \tilde{r}, \tilde{p}\right),
$$

где $|\tilde{p}|,|\tilde{r}|<1$, удовлетворяет тем же самым трем уравнениям и нормировке, что и (2.20). Поэтому эти функции совпадают, и их равенство составляет один из законов модулярных преобразований для эллиптической гамма-функции, связанный с $S L(3 ; \mathbb{Z})$-группой [25]. Из выражения $(2.21)$ следует, что $G(u ; \boldsymbol{\omega})$ является мероморфной функцией $u$ при $\omega_{1} / \omega_{2}>0$, т. е. $|q|=1$.

Благодаря соотношению $B_{3,3}\left(\sum_{k=1}^{3} \omega_{k}-u \mid \boldsymbol{\omega}\right)=-B_{3,3}(u \mid \boldsymbol{\omega})$, формула отражения для $G$-функции имеет вид $G(a ; \boldsymbol{\omega}) G(b ; \boldsymbol{\omega})=1, a+b=\sum_{k=1}^{3} \omega_{k}$. При $|q|<1$ в пределе $p, r \rightarrow 0$ (т. е. $\left.\operatorname{Im}\left(\omega_{3} / \omega_{1}\right), \quad \operatorname{Im}\left(\omega_{3} / \omega_{2}\right) \rightarrow+\infty\right)$ выражение (2.20) очевидным образом переходит в модифицированную $q$-гаммафункцию $\gamma\left(u ; \omega_{1}, \omega_{2}\right)$. Представление (2.21) дает альтернативный способ редукции к этой функции (такой предельный переход строго обоснован другим способом в [18]). Как следует из результатов [28], для определенной области значений параметров функция $G(u ; \boldsymbol{\omega})$ сходится в этом пределе к $\gamma\left(u ; \omega_{1}, \omega_{2}\right)$ экспоненциально быстро и равномерно на компактных подмножествах этой области. Этот результат важен для строгого обоснования соответствующего вырождения эллиптических гипергеометрических интегралов.

Воспользовавшись факторизацией тэта-функции (2.16), можно рассмотреть уравнение (2.17) как композицию четырех уравнений для $\Gamma_{3}(u ; \boldsymbol{\omega})$ с различными аргументами и квазипериодами. Это позволяет представить эллиптическую гамма-функцию как отношение четырех гамма-функций Барнса третьего порядка [26]

$$
\Gamma\left(e^{2 \pi i u} ; e^{2 \pi i \tau}, e^{2 \pi i \sigma}\right)=\frac{e^{-\frac{\pi i}{3} B_{3,3}(u \mid 1, \sigma, \tau)} \Gamma_{3}(u ; 1, \sigma, \tau) \Gamma_{3}(1-u ; 1,-\sigma,-\tau)}{\Gamma_{3}(1+\sigma+\tau-u ; 1, \sigma, \tau) \Gamma_{3}(u-\sigma-\tau ; 1,-\sigma,-\tau)} .
$$


При $0<\operatorname{Im}(u)<\operatorname{Im}(\tau+\sigma)$ имеет место представление

$$
\Gamma\left(e^{2 \pi i u} ; e^{2 \pi i \tau}, e^{2 \pi i \sigma}\right)=\exp \left(-\frac{i}{2} \sum_{k=1}^{\infty} \frac{\sin (\pi k(2 u-\tau-\sigma))}{k \sin (\pi k \tau) \sin (\pi k \sigma)}\right),
$$

через которое эллиптическая гамма-функция появилась в скрытой форме в работе Бакстера по восьмивершинной модели [6] (см. также [5], [25]).

\section{3. Эллиптический бета-интеграл}

В качестве первого примера эллиптических гипергеометрических функций опишем эллиптический бета-интеграл, открытый автором в [29].

Теорема 1. Рассмотрим шесть комплексных параметров $t_{j}, j=1, \ldots, 6$, и две базисные переменные $p$ u $q$, удовлетворяющие ограничениям $|p|,|q|$, $\left|t_{j}\right|<1 u \prod_{j=1}^{6} t_{j}=p q$ (условие балансировки). Тогда справедливо равенство

$$
\kappa \int_{\mathbb{T}} \frac{\prod_{j=1}^{6} \Gamma\left(t_{j} z^{ \pm 1} ; p, q\right)}{\Gamma\left(z^{ \pm 2} ; p, q\right)} \frac{d z}{z}=\prod_{1 \leqslant j<k \leqslant 6} \Gamma\left(t_{j} t_{k} ; p, q\right),
$$

где $\mathbb{T}$ обозначает положительно ориентированную единичную окружность и

$$
\kappa=\frac{(p ; p)_{\infty}(q ; q)_{\infty}}{4 \pi i} .
$$

Здесь и ниже мы пользуемся компактными обозначениями

$$
\begin{gathered}
\Gamma\left(a_{1}, \ldots, a_{k} ; p, q\right):=\Gamma\left(a_{1} ; p, q\right) \cdots \Gamma\left(a_{k} ; p, q\right), \\
\Gamma\left(t z^{ \pm 1} ; p, q\right):=\Gamma(t z ; p, q) \Gamma\left(t z^{-1} ; p, q\right), \quad \Gamma\left(z^{ \pm 2} ; p, q\right):=\Gamma\left(z^{2} ; p, q\right) \Gamma\left(z^{-2} ; p, q\right) .
\end{gathered}
$$

ДоказАтельство. Возьмем переменные $z, q, p \in \mathbb{C},|q|,|p|<1$, и пять комплексных параметров $t_{m}, m=1, \ldots, 5$, и составим функцию

$$
\rho\left(z, t_{1}, \ldots, t_{5}\right)=\frac{\prod_{m=1}^{5} \Gamma\left(t_{m} z^{ \pm 1}, A t_{m}^{-1} ; p, q\right)}{\Gamma\left(z^{ \pm 2}, A z^{ \pm 1} ; p, q\right) \prod_{1 \leqslant m<s \leqslant 5} \Gamma\left(t_{m} t_{s} ; p, q\right)},
$$

где $A=\prod_{m=1}^{5} t_{m}$. Эта функция имеет последовательности полюсов, сходящихся к нулю по точкам

$$
\mathscr{P}=\left\{t_{m} q^{a} p^{b}, A^{-1} q^{a+1} p^{b+1}\right\}_{m=1, \ldots, 5, a, b=0,1, \ldots}
$$

и уходящих на бесконечность по их $z \rightarrow 1 / z$ отражениям $\mathscr{P}^{-1}$. Обозначим через $C$ положительно ориентированный контур на комплексной плоскости, разделяющий множества $\mathscr{P}$ и $\mathscr{P}-1$ (существование такого контура - единственное ограничение на параметры $\left.t_{m}\right)$. Например, при $\left|t_{m}\right|<1,|p q|<|A|$ контур $C$ может совпадать с единичной окружностью $\mathbb{T}$. Докажем теперь, что

$$
\int_{C} \rho\left(z, t_{1}, \ldots, t_{5}\right) \frac{d z}{z}=\frac{4 \pi i}{(q ; q)_{\infty}(p ; p)_{\infty}}
$$

откуда необходимая формула будет следовать после подстановки $A=p q / t_{6}$. 
Первый шаг состоит в выводе следующего q-разностного уравнения для ядра:

$$
\rho\left(z, q t_{1}, t_{2}, \ldots, t_{5}\right)-\rho\left(z, t_{1}, \ldots, t_{5}\right)=g\left(q^{-1} z, t_{1}, \ldots, t_{5}\right)-g\left(z, t_{1}, \ldots, t_{5}\right),
$$

где

$$
g\left(z, t_{1}, \ldots, t_{5}\right)=\rho\left(z, t_{1}, \ldots, t_{5}\right) \frac{\prod_{m=1}^{5} \theta\left(t_{m} z ; p\right)}{\prod_{m=2}^{5} \theta\left(t_{1} t_{m} ; p\right)} \frac{\theta\left(t_{1} A ; p\right)}{\theta\left(z^{2}, A z ; p\right)} \frac{t_{1}}{z} .
$$

После деления уравнения (3.4) на $\rho\left(z, t_{1}, \ldots, t_{5}\right)$, оно принимает вид

$$
\begin{aligned}
& \frac{\theta\left(t_{1} z, t_{1} z^{-1} ; p\right)}{\theta\left(A z, A z^{-1} ; p\right)} \prod_{m=2}^{5} \frac{\theta\left(A t_{m}^{-1} ; p\right)}{\theta\left(t_{1} t_{m} ; p\right)}-1 \\
& \quad=\frac{t_{1} \theta\left(t_{1} A ; p\right)}{z \theta\left(z^{2} ; p\right) \prod_{m=2}^{5} \theta\left(t_{1} t_{m} ; p\right)}\left(\frac{z^{4} \prod_{m=1}^{5} \theta\left(t_{m} z^{-1} ; p\right)}{\theta\left(A z^{-1} ; p\right)}-\frac{\prod_{m=1}^{5} \theta\left(t_{m} z ; p\right)}{\theta(A z ; p)}\right) .
\end{aligned}
$$

Обе стороны этого равенства определяют эллиптические функции $\log z$ (т. е. они инвариантны относительно замены $z \rightarrow p z)$ с одинаковыми множествами полюсов и вычетами в них. Например,

$$
\lim _{z \rightarrow A} \theta\left(A z^{-1} ; p\right)\left(\begin{array}{c}
\text { левая } \\
\text { часть }
\end{array}\right)=\frac{\theta\left(t_{1} A, t_{1} A^{-1} ; p\right)}{\theta\left(A^{2} ; p\right)} \prod_{m=2}^{5} \frac{\theta\left(A t_{m}^{-1} ; p\right)}{\theta\left(t_{1} t_{m} ; p\right)},
$$

с таким же результатом для правой части. Поэтому разность выражений в двух частях равенства (3.6) определяет эллиптическую функцию без полюсов, т. е. постоянную. Эта постоянная равна нулю, так как уравнение (3.6) проверяется тривиальным образом при выборе $z=t_{1}$.

Теперь мы интегрируем (3.4) по переменной $z \in C$ и получаем

$$
I\left(q t_{1}, t_{2}, \ldots, t_{5}\right)-I\left(t_{1}, \ldots, t_{5}\right)=\left(\int_{q^{-1} C}-\int_{C}\right) g\left(z, t_{1}, \ldots, t_{5}\right) \frac{d z}{z},
$$

где $I\left(t_{1}, \ldots, t_{5}\right)=\int_{C} \rho\left(z, t_{1}, \ldots, t_{5}\right) \frac{d z}{z}$, а $q^{-1} C$ обозначает контур $C$, растянутый относительно точки $z=0$ множителем $q^{-1}$. Функция (3.5) имеет последовательности полюсов, сходящиеся к нулю по точкам $z=\left\{t_{m} q^{a} p^{b}, A^{-1} q^{a} p^{b+1}\right\}$ и уходящие на бесконечность при $z=\left\{t_{m}^{-1} q^{-a-1} p^{-b}, A q^{-a-1} p^{-b-1}\right\}$ для $m=$ $1, \ldots, 5$ и $a, b=0,1, \ldots$. Для $C=\mathbb{T}$ видно, что при $\left|t_{m}\right|<1$ и $|p|<|A|$ в кольце $1 \leqslant|z| \leqslant|q|^{-1}$ нет полюсов. Поэтому в (3.7) мы можем деформировать $q^{-1} \mathbb{T}$ в $\mathbb{T}$ и получить нуль в правой части. В результате $I\left(q t_{1}, t_{2}, \ldots, t_{5}\right)=I\left(t_{1}, \ldots, t_{5}\right)$.

Потребовав $|p|,|q|<|A|$, по симметрии между $p$ и $q$ имеем $I\left(p t_{1}, t_{2}, \ldots, t_{5}\right)=$ $I\left(t_{1}, \ldots, t_{5}\right)$. Дальнейшие замены $t_{1} \rightarrow q^{ \pm 1} t_{1}$ и $t_{1} \rightarrow p^{ \pm 1} t_{1}$ могут быть произведены, только если они не выводят параметры за пределы кольца аналитичности функции $I\left(t_{1}, \ldots, t_{5}\right)$.

Временно предположим, что $p$ и $q$ действительны, $p<q$ и $p^{n} \neq q^{k}$ для любых $n, k=0,1, \ldots$. Наложим также условие, что аргументы $t_{m}^{ \pm 1}, m=1, \ldots, 5$, и $A^{ \pm 1}$ отличаются друг от друга. Пусть теперь $C$ будет контуром, содержащим 
внутри себя $\mathscr{P}$ и два отрезка $c_{1}=\left[t_{1}, t_{1} p^{2}\right], c_{2}=\left[(p q / A) p^{-2}, p q / A\right]$ и исключающим их $z \rightarrow 1 / z$ партнеров. Тогда мы будем проводить преобразования $t_{1} \rightarrow t_{1} q^{k}, k=1,2, \ldots$, до тех пор пока $t_{1} q^{k}$ не войдет в интервал $\left[t_{1} p, t_{1} p^{2}\right]$, после чего делаем замену $t_{1} \rightarrow t_{1} p^{-1}$; это не выводит необходимые параметры за интервалы $c_{1}$ или $c_{2}$. Таким образом, мы получим $I\left(q^{j} p^{-k} t_{1}, t_{2}, \ldots, t_{5}\right)=$ $I\left(t_{1}, \ldots, t_{5}\right)$ для всех $j, k=0,1,2, \ldots$ таких, что $q^{j} p^{-k} \in[1, p]$. Поскольку такое множество точек плотно, мы приходим к выводу, что $I$ не зависит от $t_{1}$ и по симметрии от всех $t_{m}$.

Альтернативно, мы можем воспользоваться $p$-разложением $I\left(t_{1}, \ldots, t_{5}\right)=$ $\sum_{n=0}^{\infty} I_{n}\left(t_{1}, \ldots, t_{5}\right) p^{n}$ и проверить равенства $I_{n}\left(q t_{1}, \ldots, t_{5}\right)=I_{n}\left(t_{1}, \ldots, t_{5}\right)$ почленно. Коэффициенты $I_{n}$ аналитичны по параметрам вблизи точек $t_{m}=0$ (ограничения на абсолютные значения параметров снизу возникают только из требования сходимости $p$-разложения). Поэтому мы можем итерировать растяжения $t_{1} \rightarrow q t_{1}$ до достижения предельной точки. В результате, $I_{n}$ и сам интеграл $I$ не зависят от $t_{1}$ и, следовательно, от любого из $t_{m}$.

Итак, интеграл $I$ есть постоянная, зависящая только от $p$ и $q$. Для того чтобы найти ее значение, равное правой части (3.3), достаточно рассмотреть предел параметров $t_{1} t_{2} \rightarrow 1$. В этом случае два полюса приближаются к контуру интегрирования и необходимо деформировать этот контур так, чтобы пересечь их. При этом оказывается, что в пределе $t_{1} t_{2} \rightarrow 1$ только вычеты полюсов имеют конечное значение, а сам интеграл обращается в нуль. (Более подробно эта процедура описана ниже.) После доказательства формулы интегрирования в ограниченной области значений параметров ее можно аналитически продолжить в область, допускаемую контуром интегрирования $C$.

Существует множество способов вырождения эллиптического бета-интеграла. В простейшем случае необходимо подставить $t_{6}=p q /\left(t_{1} \cdots t_{5}\right)$, воспользоваться формулой отражения для $\Gamma(z ; p, q)$ и перейти к пределу $p \rightarrow 0$. При этом эллиптический бета-интеграл переходит в “тригонометрический" q-бета-интеграл Рахмана [39] (связанный с интегральным представлением для ${ }_{8} \varphi_{7}$-ряда [40]):

$$
\begin{aligned}
& \frac{(q ; q)_{\infty}}{4 \pi i} \int_{\mathbb{T}} \frac{\left(z^{2} ; q\right)_{\infty}\left(z^{-2} ; q\right)_{\infty}(A z ; q)_{\infty}\left(A z^{-1} ; q\right)_{\infty}}{\prod_{m=1}^{5}\left(t_{m} z ; q\right)_{\infty}\left(t_{m} z^{-1} ; q\right)_{\infty}} \frac{d z}{z} \\
& \quad=\frac{\prod_{m=1}^{5}\left(A t_{m}^{-1} ; q\right)_{\infty}}{\prod_{1 \leqslant m<s \leqslant 5}\left(t_{m} t_{s} ; q\right)_{\infty}},
\end{aligned}
$$

где $A=\prod_{m=1}^{5} t_{m},\left|t_{m}\right|<1$. Дальнейшее упрощение этого равенства переходом к пределу $t_{5} \rightarrow 0$ приводит к знаменитому интегралу Аски-Вильсона

$$
\frac{(q ; q)_{\infty}}{4 \pi i} \int_{\mathbb{T}} \frac{\left(z^{2} ; q\right)_{\infty}\left(z^{-2} ; q\right)_{\infty}}{\prod_{m=1}^{4}\left(t_{m} z ; q\right)_{\infty}\left(t_{m} z^{-1} ; q\right)_{\infty}} \frac{d z}{z}=\frac{\left(t_{1} t_{2} t_{3} t_{4} ; q\right)_{\infty}}{\prod_{1 \leqslant m<s \leqslant 4}\left(t_{m} t_{s} ; q\right)_{\infty}},
$$

служащему мерой для многочленов Аски-Вильсона [41] - наиболее общих ортогональных многочленов, обладающих классическими свойствами. Первое доказательство формулы (3.1) было основано на эллиптическом расширении подхода Аски [42] к вычислению интеграла Рахмана. Здесь мы представили 
доказательство, полученное в работе [43], которое обобщает метод вычисления интеграла Аски-Вильсона из [44].

Если в приведенных $q$-бета-интегралах выразить бесконечные произведения $(a ; q)_{\infty}$ через $q$-гамма-функцию Джексона и перейти к пределу $q \rightarrow 1$, то появятся "рациональные" бета-интегралы по некомпактным контурам, содержащие обычную гамма-функцию Эйлера [1]. Их дальнейшее упрощение специальным выбором параметров приводит к классическому бета-интегралу Эйлера

$$
B(\alpha, \beta)=\int_{0}^{1} t^{\alpha-1}(1-t)^{\beta-1} d t=\frac{\Gamma(\alpha) \Gamma(\beta)}{\Gamma(\alpha+\beta)}, \quad \operatorname{Re}(\alpha), \operatorname{Re}(\beta)>0 .
$$

Таким образом, эллиптический бета-интеграл (3.1) дает самую общую (из известных) точную формулу однократного интегрирования, включающую в себя бета-интеграл Эйлера как частный случай.

СлЕдСтвиЕ 2. Справедлива следующая формула суммирования ФренкеляТураева [8]:

$$
\sum_{n=0}^{N} \frac{\theta\left(t_{5}^{2} q^{2 n} ; p\right)}{\theta\left(t_{5}^{2} ; p\right)} \prod_{m=0}^{5} \frac{\theta\left(t_{m} t_{5}\right)_{n}}{\theta\left(q t_{m}^{-1} t_{5}\right)_{n}} q^{n}=\frac{\theta\left(q t_{5}^{2}, q /\left(t_{1} t_{2}\right), q /\left(t_{1} t_{3}\right), q /\left(t_{2} t_{3}\right)\right)_{N}}{\theta\left(q /\left(t_{1} t_{2} t_{3} t_{5}\right), q t_{5} / t_{1}, q t_{5} / t_{2}, q t_{5} / t_{3}\right)_{N}}
$$

где $t_{4} t_{5}=q^{-N}, \prod_{m=0}^{5} t_{m}=q$ и использовано компактное обозначение

$$
\theta\left(t_{1}, \ldots, t_{k}\right)_{n}:=\prod_{j=1}^{k} \theta\left(t_{j}\right)_{n}
$$

для произведений эллиптического символа Похгаммера

$$
\theta(t)_{n}=\prod_{j=0}^{n-1} \theta\left(t q^{j} ; p\right)=\frac{\Gamma\left(t q^{n} ; p, q\right)}{\Gamma(t ; p, q)}
$$

ДоказАтельство. Заменим в интеграле (3.1) $\mathbb{T}$ на контур $C$, отделяющий последовательности полюсов $z=t_{j} q^{a} p^{b}, j=1, \ldots, 6, a, b=0,1,2, \ldots$, сходящихся к нулю, от их $z \rightarrow 1 / z$ партнеров, стремящихся к бесконечности. Это позволяет снять ограничения $\left|t_{j}\right|<1$ без изменения правой части (3.1). Подставим теперь $t_{6}=p q / A, A=\prod_{m=1}^{5} t_{m}$, и предположим, что $\left|t_{m}\right|<1, m=1, \ldots, 4$, $\left|p t_{5}\right|<1<\left|t_{5}\right|,|p q|<|A|$ и что аргументы $t_{m}, m=1, \ldots, 5$, и $p, q$ линейно независимы над $\mathbb{Z}$. Тогда справедливо следующее равенство [32]:

$$
\kappa \int_{C} \Delta_{E}(z, \underline{t}) \frac{d z}{z}=\kappa \int_{\mathbb{T}} \Delta_{E}(z, \underline{t}) \frac{d z}{z}+c_{0}(\underline{t}) \sum_{\left|t_{5} q^{n}\right|>1, n \geqslant 0} \nu_{n}(\underline{t}),
$$

где

$$
\Delta_{E}(z, \underline{t})=\prod_{m=1}^{5} \Gamma\left(t_{m} z^{ \pm 1} ; p, q\right) / \Gamma\left(z^{ \pm 2}, A z^{ \pm 1} ; p, q\right)
$$

и

$$
c_{0}(\underline{t})=\frac{\prod_{m=1}^{4} \Gamma\left(t_{m} t_{5}^{ \pm 1} ; p, q\right)}{\Gamma\left(t_{5}^{-2}, A t_{5}^{ \pm 1} ; p, q\right)}, \quad \nu_{n}(\underline{t})=\frac{\theta\left(t_{5}^{2} q^{2 n} ; p\right)}{\theta\left(t_{5}^{2} ; p\right)} \prod_{m=0}^{5} \frac{\theta\left(t_{m} t_{5}\right)_{n}}{\theta\left(q t_{m}^{-1} t_{5}\right)_{n}} q^{n}
$$


Здесь мы ввели новый параметр $t_{0}$ с помощью соотношения $\prod_{m=0}^{5} t_{m}=q$. Множитель $\kappa$ отсутствует в коэффициенте $c_{0}$ ввиду $\lim _{z \rightarrow 1}(1-z) \Gamma(z ; p, q)=$ $1 /\left((p ; p)_{\infty}(q ; q)_{\infty}\right)$ и удвоения числа вычетов (последнее вытекает из симметрии ядра $\left.z \rightarrow z^{-1}\right)$.

В пределе $t_{5} t_{4} \rightarrow q^{-N}, N=0,1,2, \ldots$, интеграл в левой части (3.9) (совпадающий с $(3.1))$ и множитель $c_{0}(\underline{t})$ перед суммой вычетов в правой части расходятся. При этом интеграл по единичной окружности $\mathbb{T}$ в правой части остается конечным. Разделив все члены на $c_{0}(\underline{t})$ и перейдя к предельному равенству, мы получаем формулу суммирования (3.8), которая впервые была получена в [8] совершенно иным способом.

Другие доказательства формулы (3.8) приведены в [3], [45]-[49]. При $p \rightarrow 0$ и фиксированных параметрах равенство (3.8) редуцируется к сумме Джексона для обрывающегося совершенно уравновешенного сбалансированного $q$-гипергеометрического ряда $8 \varphi_{7}$ [1]. Таким образом, левая часть формулы (3.8) представляет собой эллиптический аналог этого $q$-ряда.

Используя модифицированную эллиптическую гамма-функцию, нетрудно построить модифицированный эллиптический бета-интеграл [38], у которого один из базисных параметров может лежать на единичной окружности, скажем, $|q|=1$.

TеОрема 3. Пусть $\operatorname{Im}\left(\omega_{1} / \omega_{2}\right) \geqslant 0, \operatorname{Im}\left(\omega_{3} / \omega_{1}\right)>0, \operatorname{Im}\left(\omega_{3} / \omega_{2}\right)>0$ u uесть параметров $g_{j} \in \mathbb{C}, j=1, \ldots, 6$, удовлетворяют ограничениям $\operatorname{Im}\left(g_{j} / \omega_{3}\right)<0$ $u \sum_{j=1}^{6} g_{j}=\sum_{k=1}^{3} \omega_{k}$. Тогдa

$$
\tilde{\kappa} \int_{-\omega_{3} / 2}^{\omega_{3} / 2} \frac{\prod_{j=1}^{6} G\left(g_{j} \pm u ; \boldsymbol{\omega}\right)}{G( \pm 2 u ; \boldsymbol{\omega})} \frac{d u}{\omega_{2}}=\prod_{1 \leqslant j<m \leqslant 6} G\left(g_{j}+g_{m} ; \boldsymbol{\omega}\right),
$$

əдe

$$
\tilde{\kappa}=-\frac{(q ; q)_{\infty}(p ; p)_{\infty}(r ; r)_{\infty}}{2(\tilde{q} ; \tilde{q})_{\infty}} .
$$

Здесъ интегрирование идет вдоль отрезка с граничными точками - $\omega_{3} / 2$ и $\omega_{3} / 2$ и используется соглашение $G(a \pm b ; \boldsymbol{\omega}) \equiv G(a+b ; \boldsymbol{\omega}) G(a-b ; \boldsymbol{\omega})$.

ДокАЗАТЕЛЬство. Подставляя соотношение (2.21) в левую часть (3.10), получим

$$
\tilde{\kappa} e^{\pi i a / 3} \int_{-\omega_{3} / 2}^{\omega_{3} / 2} \frac{\prod_{j=1}^{6} \Gamma\left(e^{-2 \pi i\left(g_{j} \pm u\right) / \omega_{3}} ; \tilde{r}, \tilde{p}\right)}{\Gamma\left(e^{\mp 4 \pi i u / \omega_{3}} ; \tilde{r}, \tilde{p}\right)} \frac{d u}{\omega_{2}},
$$

где $a=2 B_{3,3}(0 \mid \omega)-2 \sum_{j=1}^{6} B_{3,3}\left(g_{j} \mid \omega\right)$. Ограничения на параметры позволяют использовать формулу (3.1) с подстановками

$$
z \rightarrow e^{\frac{2 \pi i}{\omega_{3}} u}, \quad t_{j} \rightarrow e^{-\frac{2 \pi i}{\omega_{3}} g_{j}}, \quad p \rightarrow e^{-2 \pi i \frac{\omega_{1}}{\omega_{3}}}, \quad q \rightarrow e^{-2 \pi i \frac{\omega_{2}}{\omega_{3}}},
$$

что для (3.11) приводит к

$$
\begin{aligned}
& \frac{2 \tilde{\kappa} \omega_{3} \omega_{2}^{-1} e^{\pi i a / 3}}{(\tilde{r} ; \tilde{r})_{\infty}(\tilde{p} ; \tilde{p})_{\infty}} \prod_{1 \leqslant j<m \leqslant 6} \Gamma\left(e^{-2 \pi i\left(g_{j}+g_{m}\right) / \omega_{3}} ; \tilde{r}, \tilde{p}\right) \\
& \quad=\frac{2 \tilde{\kappa} \omega_{3} \omega_{2}^{-1} e^{\pi i(a+b) / 3}}{(\tilde{r} ; \tilde{r})_{\infty}(\tilde{p} ; \tilde{p})_{\infty}} \prod_{1 \leqslant j<m \leqslant 6} G\left(g_{j}+g_{m} ; \boldsymbol{\omega}\right),
\end{aligned}
$$


где $b=\sum_{1 \leqslant j<m \leqslant 6} B_{3,3}\left(g_{j}+g_{m} \mid \omega\right)$. Непосредственное вычисление показывает, что

$$
a+b=\frac{1}{4}\left(\sum_{k=1}^{3} \omega_{k}\right)\left(\sum_{k=1}^{3} \omega_{k}^{-1}\right) .
$$

Поэтому при выборе

$$
\tilde{\kappa}^{-1}=\frac{2 \omega_{3} e^{\frac{\pi i}{12}\left(\sum_{k=1}^{3} \omega_{k}\right)\left(\sum_{k=1}^{3} \omega_{k}^{-1}\right)}}{\omega_{2}(\tilde{r} ; \tilde{r})_{\infty}(\tilde{p} ; \tilde{p})_{\infty}}
$$

получаем необходимый ответ. После применения закона модулярного преобразования для функции Дедекинда

$$
e^{-\frac{\pi i}{12 \tau}}\left(e^{-2 \pi i / \tau} ; e^{-2 \pi i / \tau}\right)_{\infty}=(-i \tau)^{1 / 2} e^{\frac{\pi i \tau}{12}}\left(e^{2 \pi i \tau} ; e^{2 \pi i \tau}\right)_{\infty}
$$

к бесконечным произведениям, входящим в $\tilde{\kappa}$, получаем

$$
\tilde{\kappa}^{-1}=-2 \sqrt{\frac{\omega_{1}}{i \omega_{2}}} \frac{e^{\frac{\pi i}{12}\left(\frac{\omega_{1}}{\omega_{2}}+\frac{\omega_{2}}{\omega_{1}}\right)}}{(r ; r)_{\infty}(p ; p)_{\infty}} .
$$

Еще одно применение соотношения (3.12) позволяет заменить экспоненциальную функцию отношением бесконечных произведений, что и приводит к необходимой форме $\kappa$.

Если взять предел $\operatorname{Im}\left(\omega_{3}\right) \rightarrow \infty$ таким образом, что $p, r \rightarrow 0$, то модифицированный эллиптический бета-интеграл редуцируется к $q$-бета-интегралу типа Меллина-Барнса. Точнее, для $\omega_{1,2}$ таких, что $\operatorname{Im}\left(\omega_{1} / \omega_{2}\right) \geqslant 0$ и $\operatorname{Re}\left(\omega_{1} / \omega_{2}\right)>0$, мы подставляем $g_{6}=\sum_{k=1}^{3} \omega_{k}-\mathscr{A}$, где $\mathscr{A}=\sum_{j=1}^{5} g_{j}$, и применяем формулу инверсии для $G(u ; \boldsymbol{\omega})$. После этого мы полагаем $\omega_{3}=i t \omega_{2}, t \rightarrow+\infty$, и формально получаем

$$
\int_{\mathbb{L}} \frac{\prod_{j=1}^{5} \gamma\left(g_{j} \pm u ; \boldsymbol{\omega}\right)}{\gamma( \pm 2 u, \mathscr{A} \pm u ; \boldsymbol{\omega})} \frac{d u}{\omega_{2}}=-2 \frac{(\tilde{q} ; \tilde{q})_{\infty}}{(q ; q)_{\infty}} \frac{\prod_{1 \leqslant j<m \leqslant 5} \gamma\left(g_{j}+g_{m} ; \boldsymbol{\omega}\right)}{\prod_{j=1}^{5} \gamma\left(\mathscr{A}-g_{j} ; \boldsymbol{\omega}\right)},
$$

где $\gamma\left(u ; \omega_{1}, \omega_{2}\right)$ обозначает модифицированную $q$-гамма-функцию, а интегрирование ведется вдоль прямой $\mathbb{L} \equiv i \omega_{2} \mathbb{R}$. При этом параметры должны удовлетворять ограничениям $\operatorname{Re}\left(g_{j} / \omega_{2}\right)>0$ и $\operatorname{Re}\left(\left(\mathscr{A}-\omega_{1}\right) / \omega_{2}\right)<1$. Эта формула интегрирования представляет собой “гиперболический” аналог интеграла Рахмана; впервые она была доказана Стокманом в [50]. Из-за некомпактности контура интегрирования описанный метод вывода (3.13) строг при условии равномерной сходимости функции $G(u ; \boldsymbol{\omega})$ к $\gamma\left(u ; \omega_{1}, \omega_{2}\right)$, что следует из результатов, полученных Рэйнсом в [28]. Можно обосновать формулу (3.13) и методом, использованным выше для доказательства эллиптического бета-интеграла. Предельный переход $q \rightarrow 1$ приводит к тому же рациональному бета-интегралу, что и "тригонометрический" интеграл Рахмана.

Подытоживая сказанное в данном разделе, мы видим, что эллиптический бета-интеграл включает в себя целую иерархию точно вычисляемых интегралов: два типа q-бета-интегралов, рациональный класс бета-интегралов, ядра которых выражаются через гамма-функцию Эйлера, и классический бета-интеграл Эйлера. Эта схема отражает общую картину вырождений эллиптических гипергеометрических функций, строго рассмотренную в [28]. 


\section{4. Общие эллиптические гипергеометрические ряды и интегралы}

В работах [10] и [11] автор предложил определения общих эллиптических гипергеометрических рядов и интегралов, которые и будут рассмотрены в данном разделе.

4.1. Эллиптический аналог функции Мейера. Однократные контурные интегралы $\int_{C} \Delta(u) d u$ называются эллиптическими гипергеометрическими интегралами, если мероморфная функция $\Delta(u)$ удовлетворяет следующей системе трех уравнений:

$$
\Delta\left(u+\omega_{k}\right)=h_{k}(u) \Delta(u), \quad k=1,2,3,
$$

где параметры $\omega_{1,2,3} \in \mathbb{C}$ линейно независимы над $\mathbb{Z}$, a $h_{k}(u)$ - некоторые эллиптические функции с периодами $\omega_{k}, \omega_{k+1}$ (мы полагаем $\omega_{k+3}=\omega_{k}$ ).

Общая эллиптическая функция порядка $s$ с периодами $\omega_{2}$ и $\omega_{3}$ может быть представлена в виде (см. приложение А)

$$
h_{1}(u)=y_{1} \prod_{j=1}^{s} \frac{\theta\left(t_{j} e^{2 \pi i u / \omega_{2}} ; p\right)}{\theta\left(w_{j} e^{2 \pi i u / \omega_{2}} ; p\right)},
$$

где $y_{1}$ - произвольная постоянная, a $t_{j}, w_{j}$ - параметры, удовлетворяющие условию балансировки $\prod_{j=1}^{s} t_{j}=\prod_{j=1}^{s} w_{j}$ (напомним, что $\left.p=e^{2 \pi i \omega_{3} / \omega_{2}}\right)$. Пользуясь свойствами функции $\Gamma(z ; p, q)$, легко построить общее решение первого $(k=1)$ уравнения в $(4.1)$ при $|q|<1$ :

$$
\Delta(u)=y_{1}^{u / \omega_{1}} \varphi(u) \prod_{j=1}^{s} \frac{\Gamma\left(t_{j} z ; p, q\right)}{\Gamma\left(w_{j} z ; p, q\right)}, \quad z=e^{2 \pi i u / \omega_{2}}
$$

где $\varphi\left(u+\omega_{1}\right)=\varphi(u)$ - произвольная периодическая функция. Таким образом, если бы мы ограничились только одним уравнением для $\Delta(u)$, то наше определение интегралов было бы в значительной степени неоднозначным.

Произвольная эллиптическая функция порядка $\ell$ с периодами $\omega_{1}$ и $\omega_{3}$ имеет вид

$$
h_{2}(u)=y_{2} \prod_{j=1}^{\ell} \frac{\theta\left(\tilde{t}_{j} e^{-2 \pi i u / \omega_{1}} ; r\right)}{\theta\left(\widetilde{w}_{j} e^{-2 \pi i u / \omega_{1}} ; r\right)},
$$

где $|r|<1, y_{2}$ - произвольная постоянная, а параметры удовлетворяют соотношению $\prod_{j=1}^{\ell} \tilde{t}_{j}=\prod_{j=1}^{\ell} \widetilde{w}_{j}$. Уравнение из (4.1) с $k=2$ является теперь ограничением на функцию $\varphi(u)$. Легко доказать, что общее решение этих двух уравнений при $|q|<1$ имеет вид

$$
\Delta(u)=\phi(u) \prod_{j=1}^{s} \frac{\Gamma\left(t_{j} e^{2 \pi i u / \omega_{2}} ; p, q\right)}{\Gamma\left(w_{j} e^{2 \pi i u / \omega_{2}} ; p, q\right)} \prod_{j=1}^{\ell} \frac{\Gamma\left(\tilde{t}_{j} e^{-2 \pi i u / \omega_{1}} ; \tilde{q}, r\right)}{\Gamma\left(\widetilde{w}_{j} e^{-2 \pi i u / \omega_{1}} ; \tilde{q}, r\right)},
$$

где $\phi(u)$ - произвольная функция, удовлетворяющая уравнениям $\phi\left(u+\omega_{1}\right)=$ $y_{1} \phi(u)$ и $\phi\left(u+\omega_{2}\right)=y_{2} \phi(u)$. Таким образом, $\phi(u)$ есть мероморфная тэта-функция со специальными множителями квазипериодичности, общий вид которой 
легко установить (см. приложение А):

$$
\phi(u)=e^{c u+d} \prod_{k=1}^{m} \frac{\theta\left(a_{k} e^{2 \pi i u / \omega_{2}} ; q\right)}{\theta\left(b_{k} e^{2 \pi i u / \omega_{2}} ; q\right)}=e^{c u+d} \prod_{k=1}^{m} \frac{\Gamma\left(p a_{k} e^{2 \pi i u / \omega_{2}}, b_{k} e^{2 \pi i u / \omega_{2}} ; p, q\right)}{\Gamma\left(a_{k} e^{2 \pi i u / \omega_{2}}, p b_{k} e^{2 \pi i u / \omega_{2}} ; p, q\right)},
$$

где $m$ - произвольное целое число, параметр $d$ произволен, а параметры $a_{k}, b_{k}, c$ связаны с $y_{1}$ и $y_{2}$ соотношениями $y_{2}=e^{c \omega_{2}}$ и $y_{1}=e^{c \omega_{1}} \prod_{k=1}^{m} b_{k} a_{k}^{-1}$.

Благодаря представлению через эллиптические гамма-функции, функцию $\phi(u)$ можно свести к чисто экспоненциальному множителю заменой $s$ в $\Delta(u)$ на $s+2 m$ и выбором параметров $t_{s+k}=p a_{k}, t_{s+m+k}=b_{k}, w_{s+k}=a_{k}, w_{s+m+k}=$ $p b_{k}, k=1, \ldots, m$, что не нарушает условия балансировки $\prod_{j=1}^{s+2 m} t_{j}=\prod_{j=1}^{s+2 m} w_{j}$. Поскольку $s$ было произвольным с самого начала, не ограничивая общности, можем считать $\phi(u)=e^{c u+d}$.

Таким образом, два уравнения уже фиксируют вид ядра $\Delta(u)$, т. е. для эллиптической функции $h_{3}(u)$ автоматически получаем

$$
h_{3}(u)=e^{c \omega_{3}} \prod_{j=1}^{s} \frac{\theta\left(t_{j} e^{2 \pi i u / \omega_{2}} ; q\right)}{\theta\left(w_{j} e^{2 \pi i u / \omega_{2}} ; q\right)} \prod_{j=1}^{\ell} \frac{\theta\left(r^{-1} \widetilde{w}_{j} e^{-2 \pi i u / \omega_{1}} ; \tilde{q}\right)}{\theta\left(r^{-1} \tilde{t}_{j} e^{-2 \pi i u / \omega_{1}} ; \tilde{q}\right)} .
$$

Итак, при $|q|<1$ наиболее общий эллиптический гипергеометрический интеграл имеет вид [11], [31]

$$
\int_{C} e^{c u+d} \prod_{j=1}^{s} \frac{\Gamma\left(t_{j} e^{2 \pi i u / \omega_{2}} ; p, q\right)}{\Gamma\left(w_{j} e^{2 \pi i u / \omega_{2}} ; p, q\right)} \prod_{j=1}^{\ell} \frac{\Gamma\left(\tilde{t}_{j} e^{-2 \pi i u / \omega_{1}} ; \tilde{q}, r\right)}{\Gamma\left(\widetilde{w}_{j} e^{-2 \pi i u / \omega_{1}} ; \tilde{q}, r\right)} d u
$$

с указанными выше двумя условиями балансировки для параметров и некоторым контуром интегрирования $C$.

Рассмотрим определение интегралов при $|q|=1$. Оказывается, что теперь даже функция $h_{2}(u)$ не может быть произвольной. В этом случае необходимо выбрать $\ell=s$ и подобрать параметры $t_{j}, \tilde{t}_{j}$ и $w_{j}, \widetilde{w}_{j}$ так, чтобы все Г-функции комбинировались в модифицированные эллиптические гамма-функции $G(u ; \boldsymbol{\omega})$ (именно таким образом она и была построена в [11]). Это приводит к интегралам вида

$$
\int_{C} e^{c u+d} \prod_{j=1}^{s} \frac{G\left(u+g_{j} ; \boldsymbol{\omega}\right)}{G\left(u+v_{j} ; \boldsymbol{\omega}\right)} d u,
$$

где параметры $g_{j}$ и $v_{j}$ удовлетворяют условию балансировки $\sum_{j=1}^{s}\left(g_{j}-v_{j}\right)=0$ совместно с соотношениями $t_{j}=e^{2 \pi i g_{j} / \omega_{2}}, w_{j}=e^{2 \pi i v_{j} / \omega_{2}}, \tilde{t}_{j}=r e^{-2 \pi i g_{j} / \omega_{1}}$, $\widetilde{w}_{j}=r e^{-2 \pi i v_{j} / \omega_{1}}$, и $y_{1,2}=e^{c \omega_{1,2}}$.

Случай $|q|>1$ оказывается эквивалентным случаю $|q|<1$ после замены параметров и приводит к интегралам

$$
\int_{C} e^{c u+d} \prod_{j=1}^{s} \frac{\Gamma\left(q^{-1} w_{j} e^{2 \pi i u / \omega_{2}} ; p, q^{-1}\right)}{\Gamma\left(q^{-1} t_{j} e^{2 \pi i u / \omega_{2}} ; p, q^{-1}\right)} \prod_{j=1}^{\ell} \frac{\Gamma\left(\tilde{q}^{-1} \widetilde{w}_{j} e^{-2 \pi i u / \omega_{1}} ; \tilde{q}^{-1}, r\right)}{\Gamma\left(\tilde{q}^{-1} \tilde{t}_{j} e^{-2 \pi i u / \omega_{1}} ; \tilde{q}^{-1}, r\right)} d u .
$$

Функции (4.3), (4.4) и (4.5) можно назвать эллиптическими аналогами функции Мейера, поскольку при определенном выборе параметров и контура интегрирования $C$ они вырождаются к этой функции [12]. При этом на промежуточном 
этапе возникают различные $q$-аналоги функции Мейера, включая случаи, рассмотренные в [51]. Более общие тэта-гипергеометрические аналоги функции Мейера, для которых ядра $\Delta(u)$ удовлетворяют системе уравнений (4.1), где $h_{k}(u)$ - произвольные мероморфные тэта-функции, построены в [11]; мы их здесь не рассматриваем.

\section{2. Вполне уравновешенные и совершенно уравновешенные инте-} гралы. Рассмотрим интегралы (4.3) с $\ell=c=d=0$ и заменим переменную интегрирования на $z=e^{2 \pi i u / \omega_{2}}$. До настоящего времени мы не фиксировали контур $C$. Выберем его в виде единичной окружности $\mathbb{T}$, ориентированной против часовой стрелки. В результате мы получаем интегралы вида

$$
\int_{\mathbb{T}} \Delta(z) \frac{d z}{z}, \quad \Delta(z)=\prod_{k=1}^{s} \frac{\Gamma\left(t_{k} z ; p, q\right)}{\Gamma\left(w_{k} z ; p, q\right)},
$$

с $\prod_{k=1}^{s} t_{k}=\prod_{k=1}^{s} w_{k}$. Если выполнены условия $w_{k} t_{k}=p q, k=1, \ldots, s$, то интегралы принимают вид

$$
\int_{\mathbb{T}} \Delta^{(s)}(z) \frac{d z}{z}, \quad \Delta^{(s)}(z)=\prod_{k=1}^{s} \Gamma\left(t_{k} z, t_{k} / z ; p, q\right),
$$

и называются вполне уравновешенными. Условие балансировки для них принимает вид $\prod_{k=1}^{s} t_{k}^{2}=(p q)^{s}$ или $\prod_{k=1}^{s} t_{k}=\mu(p q)^{s / 2}$ с неопределенностью в выборе знака $\mu= \pm 1$. Формула отражения $\Gamma(a, b ; p, q)=1, a b=p q$, показывает, что выбор параметров $t_{j} t_{k}=p q$ играет существенную роль, уменьшая число параметров в $\Delta^{(s)}(z)$. В частности, при $t_{k}^{2}=p q$ переменная $t_{k}$ полностью исчезает из формул. Очевидно, что функция

$$
h^{(p)}(z):=\frac{\Delta^{(s)}(q z)}{\Delta^{(s)}(z)}=\prod_{k=1}^{s} \frac{\theta\left(t_{k} z ; p\right)}{\theta\left(p q z / t_{k} ; p\right)}
$$

является $p$-эллиптической, т. е. $h^{(p)}(p z)=h^{(p)}(z)$. Обозначив $u_{k}:=t_{k} z, v_{k}:=$ $p q z / t_{k}, \lambda:=p q z^{2}$, можно переписать $h^{(p)}(z)$ как

$$
h^{(p)}\left(u_{1}, \ldots, u_{s} ; \lambda\right)=\prod_{k=1}^{s} \frac{\theta\left(u_{k} ; p\right)}{\theta\left(v_{k} ; p\right)}
$$

с условиями вполне уравновешенности $u_{k} v_{k}=\lambda, k=1, \ldots, s$, и балансировки $\prod_{k=1}^{s} u_{k}=\mu \lambda^{s / 2}$.

Рассмотрим все возможные $p$-сдвиги параметров $u_{1}, \ldots, u_{s}$ и $\lambda$ :

$$
u_{k} \rightarrow p^{n_{k}} u_{k}, \quad \lambda \rightarrow p^{N} \lambda, \quad n_{k}, N \in \mathbb{Z},
$$

и потребуем, чтобы $h^{(p)}$ была инвариантна относительно максимально возможной группы этих преобразований. Условие балансировки приводит к ограничению $\sum_{k=1}^{s} n_{k}=s N / 2$. При $N=0$ легко проверить, что $h^{(p)}\left(\ldots, p u_{a}, \ldots\right.$, $\left.p^{-1} u_{b}, \ldots ; \lambda\right)=h^{(p)}\left(u_{1}, \ldots, u_{s} ; \lambda\right)$, т. е. $h^{(p)}$ является эллиптической функцией всех своих параметров. Преобразования с $N \neq 0$ сложнее и зависят от четности переменной $s$. Для нечетных $s$ целое число $N$ должно быть четным. 
Полная группа симметрий генерируется тогда преобразованиями с $N=0$ и, скажем, $n_{1}=s, n_{k}=0, k \neq 1$, и $N=2$, что дает

$$
\frac{h^{(p)}\left(p^{s} u_{1}, u_{2}, \ldots, u_{s} ; p^{2} \lambda\right)}{h^{(p)}\left(u_{1}, \ldots, u_{s} ; \lambda\right)}=\frac{\lambda^{s}}{\prod_{k=1}^{s} u_{k}^{2}}=1,
$$

т. е. значение $\mu$ не фиксируется.

Что же касается четных $s=2 m$, то в этом случае на $N$ нет ограничений, и достаточно рассмотреть преобразование, соответствующее выбору $n_{1}=m$, $N=1$, со всеми остальными $n_{k}=0$. Тогда имеем

$$
\frac{h^{(p)}\left(p^{m} u_{1}, u_{2}, \ldots, u_{2 m} ; p \lambda\right)}{h^{(p)}\left(u_{1}, \ldots, u_{2 m} ; \lambda\right)}=\frac{\lambda^{m}}{\prod_{k=1}^{2 m} u_{k}}=\mu .
$$

Требуя, чтобы это преобразование было симметрией, мы однозначно фиксируем условие балансировки, $\mu=1$.

Нетрудно проверить, что для произвольного $\mu= \pm 1$ верно равенство

$$
\Delta^{(s)}\left(p^{i} q^{j} z\right) \Delta^{(s)}(z)=\Delta^{(s)}\left(p^{i} z\right) \Delta^{(s)}\left(q^{j} z\right)
$$

для всех $i, j \in \mathbb{Z}$. Наоборот, из этого условия можно получить условие балансировки с $\mu= \pm 1$. Переходя к пределам $z \rightarrow \pm p^{-i / 2} q^{-j / 2}$ и используя симметрию $\Delta^{(s)}(z)=\Delta^{(s)}\left(z^{-1}\right)$, получаем $\Delta^{(s)}\left( \pm p^{i / 2} q^{j / 2}\right)^{2}=\Delta^{(s)}\left( \pm p^{i / 2} q^{-j / 2}\right)^{2}$. Прямое вычисление дает

$$
\Delta^{(s)}\left( \pm p^{i / 2} q^{j / 2}\right)=\left((\mp 1)^{s} \mu\right)^{i j} \Delta^{(s)}\left( \pm p^{i / 2} q^{-j / 2}\right) .
$$

Для четных $s=2 m$ и $\mu=1$ получаем

$$
\Delta^{(2 m)}\left( \pm p^{i / 2} q^{j / 2}\right)=\Delta^{(2 m)}\left( \pm p^{i / 2} q^{-j / 2}\right)
$$

при условии, что функции в обеих частях равенства корректно определены. Последнее требование выполняется, если мы не сталкиваемся с полюсами, т. е. $t_{r} \neq \pm p^{a / 2} q^{b / 2}, a, b \in \mathbb{Z}$, для всех $r$.

Будем называть интегралы $\int_{\mathbb{T}} \Delta(z) \frac{d z}{z}$ совершенно уравновешенными, если их ядра имеют вид

$$
\Delta_{v w p}^{(2 m+6)}(z)=\frac{\prod_{k=1}^{2 m+6} \Gamma\left(t_{k} z^{ \pm 1} ; p, q\right)}{\Gamma\left(z^{ \pm 2} ; p, q\right)}=\theta\left(z^{2} ; p\right) \theta\left(z^{-2} ; q\right) \prod_{k=1}^{2 m+6} \Gamma\left(t_{k} z^{ \pm 1} ; p, q\right) .
$$

Это ядро может быть получено из $\Delta^{(2 m+14)}(z)$ ограничением параметров

$$
t_{2 m+7}, \ldots, t_{2 m+14}=\left( \pm(p q)^{1 / 2}, \pm p^{1 / 2} q, \pm p q^{1 / 2}, \pm p q\right)
$$

это следует из формулы отражения и формулы удвоения аргумента для функции $Г(z ; p, q)$ :

$$
\Gamma\left(z^{2} ; p, q\right)=\Gamma\left( \pm z, \pm q^{1 / 2} z, \pm p^{1 / 2} z, \pm(p q)^{1 / 2} z ; p, q\right)
$$


Условие балансировки принимает теперь вид

$$
\prod_{k=1}^{2 m+6} t_{k}=\mu(p q)^{m+1}
$$

где выбор знака $\mu=1$ мы считаем каноническим, так как он приводит к дополнительным симметриям. Ядро $\Delta_{v w p}^{(2 m+6)}(z)$ также может быть получено из $\Delta^{(2 m+12)}(z)$ ограничениями $t_{2 m+7}, \ldots, t_{2 m+12}=\left( \pm p^{1 / 2} q, \pm p q^{1 / 2}, \pm p q\right)$, поскольку выбор $t_{k}^{2}=p q$ просто устраняет соответствующие Г-множители. Однако мы не будем пользоваться такой редукцией, так как для нее в условии балансировки изменится знак: $\prod_{k=1}^{2 m+6} t_{k}=-\mu(p q)^{m+1}$.

В дальнейшем, мы будем изучать совершенно уравновешенные эллиптические гипергеометрические интегралы вида

$$
I^{(m)}\left(t_{1}, \ldots, t_{2 m+6}\right)=\kappa \int_{\mathbb{T}} \frac{\prod_{k=1}^{2 m+6} \Gamma\left(t_{k} z^{ \pm 1} ; p, q\right)}{\Gamma\left(z^{ \pm 2} ; p, q\right)} \frac{d z}{z}
$$

c “правильным" условием балансировки $\prod_{k=1}^{2 m+6} t_{k}=(p q)^{m+1}$. Эти интегралы представляют собой эллиптические аналоги чисто гипергеометрических функций ${ }_{m+1} F_{m}$. В частности, при $m=0$ мы получаем эллиптический бета-интеграл. Ограничения $t_{2 m+5}=(p q)^{1 / 2}, t_{2 m+6}=-(p q)^{1 / 2}$ редуцируют эти интегралы к $I^{(m-1)}\left(t_{1}, \ldots, t_{2 m+4}\right)$ с условием балансировки $\prod_{k=1}^{2 m+4} t_{k}=-(p q)^{m}$. Появление знака “_” в правой части просто указывает, что эти интегралы на самом деле должны рассматриваться как обобщение ${ }_{m+1} F_{m^{-}}$, a не ${ }_{m} F_{m-1}$-функций. Например, такой выбор в эллиптическом бета-интеграле с учетом соотношения $\Gamma(-p q ; p, q)=2(-p ; p)_{\infty}(-q ; q)_{\infty}$ дает следующее:

$$
\begin{aligned}
& \frac{1}{2 \pi i} \int_{C} \frac{\prod_{k=1}^{4} \Gamma\left(t_{k} z^{ \pm 1} ; p, q\right)}{\Gamma\left(z^{ \pm 2} ; p, q\right)} \frac{d z}{z} \\
& \quad=4\left(p^{2} ; p^{2}\right)_{\infty}\left(q^{2} ; q^{2}\right)_{\infty} \prod_{1 \leqslant j<k \leqslant 4} \Gamma\left(t_{j} t_{k} ; p, q\right) \prod_{k=1}^{4} \Gamma\left(p q t_{k}^{2} ; p^{2}, q^{2}\right),
\end{aligned}
$$

где $\prod_{k=1}^{4} t_{k}=-1$ и контур $C$ отделяет сходящиеся к нулю последовательности полюсов ядра от уходящих на бесконечность. При $p \rightarrow 0$ получаем специальный случай интеграла Аски-Вильсона.

Множитель в ядре интегралов, появляющийся из-за ограничений совершенной уравновешенности на параметры, приводит к интересному свойству [34]

$$
\Delta_{v w p}^{(2 m+6)}\left(p^{i / 2} q^{j / 2}\right)=-\mu^{i j} \Delta_{v w p}^{(2 m+6)}\left(p^{i / 2} q^{-j / 2}\right),
$$

т. е. к знаку “_" в правой части при каноническом выборе $\mu=1$, что принципиально отличается от (4.7). Действительно, при $i=0$ или $j=0$ имеем нули в обеих частях равенства, а в других случаях получаем

$$
\frac{\lim _{z \rightarrow p^{i / 2} q^{j / 2}} \Gamma_{p, q}^{-1}\left(z^{2}, z^{-2}\right)}{\lim _{z \rightarrow p^{i / 2} q^{-j / 2}} \Gamma_{p, q}^{-1}\left(z^{2}, z^{-2}\right)}=\frac{\theta\left(p^{-i} q^{-j} ; q\right) \theta\left(p^{i} q^{j} ; p\right)}{\theta\left(p^{-i} q^{j} ; q\right) \theta\left(p^{i} q^{-j} ; p\right)}=-(p q)^{-2 i j}
$$


что вместе с описанными свойствами $\Delta^{(2 m+6)}(z)$ дает приведенную формулу. При $i, j \neq 0$ возникает некоммутативность двух пределов:

$$
\begin{aligned}
& \frac{\lim _{z \rightarrow p^{i / 2} q^{j / 2}} \Delta_{v w p}^{(2 m+6)}(z)}{\lim _{z \rightarrow p^{i / 2} q^{-j / 2}} \Delta_{v w p}^{(2 m+6)}(z)}=-\mu^{i j} \\
& \quad \neq \lim _{t_{2 m+7}, \ldots, t_{2 m+14} \rightarrow\left( \pm(p q)^{1 / 2}, \pm p^{1 / 2} q, \pm p q^{1 / 2}, \pm p q\right)} \frac{\Delta^{(2 m+14)}\left(p^{i / 2} q^{j / 2}\right)}{\Delta^{(2 m+14)}\left(p^{i / 2} q^{-j / 2}\right)}=\mu^{i j},
\end{aligned}
$$

хотя оба они корректно определены. Причиной этого противоречия служит то, что условие совершенной уравновешенности использует “запрещенные” значения параметров, приводящие к полюсам и нулям $\Delta^{(2 m+14)}(z)$ при $z=p^{i / 2} q^{j / 2}$.

Как показано в [34], произведение $\prod_{1 \leqslant j \leqslant k \leqslant m}\left(t_{j} t_{k} ; p, q\right)_{\infty} I^{(m)}(\underline{t})$ является голоморфной функцией параметров $t_{j} \in \mathbb{C}^{*}$. Дополнительно, знак “_" в правой части (4.9) гарантирует голоморфность интеграла $I^{(m)}(\underline{t})$ в точках $t_{k}^{2}=p^{-a} q^{-b}$, $a, b=0,1,2, \ldots$ Если же придать каким-либо параметрам запрещенные значения $\pm p^{a / 2} q^{b / 2}, a, b \in \mathbb{Z}$, то последнее свойство исчезнет.

4.3. Ряды. Согласно общему определению [10], формальные ряды $\sum_{n \in \mathbb{Z}} c_{n}$ называются эллиптическими гипергеометрическими рядами, если отношение соседних членов ряда $c_{n+1} / c_{n}$ является некоторой эллиптической функцией $n \in \mathbb{C}$. Это определение находится в русле идей Похгаммера и Хорна, которые используются при построении обычных и q-гипергеометрических рядов [2].

Как мы уже видели на примере суммы Френкеля-Тураева, эллиптические гипергеометрические ряды возникают как суммы вычетов определенных последовательностей полюсов ядер эллиптических гипергеометрических интегралов. Действительно, рассмотрим полюсы подынтегральной функции в (4.3), расположенные в точках $u=a+\omega_{1} n, n=0,1,2, \ldots$, для некоторого параметра $a$, и обозначим вычеты этих полюсов через $c_{n}$. При $u \rightarrow a+\omega_{1} n$ имеем $\Delta(u) \rightarrow c_{n} /\left(u-a-\omega_{1} n\right)+O(1)$. Теперь несложно заметить, что отношение

$$
\lim _{u \rightarrow a+\omega_{1} n} \frac{\Delta\left(u+\omega_{1}\right)}{\Delta(u)}=\frac{c_{n+1}}{c_{n}}=\lim _{u \rightarrow a+\omega_{1} n} h_{1}(u)=h_{1}\left(a+\omega_{1} n\right)
$$

является эллиптической функцией $n$ с периодами $\omega_{2} / \omega_{1}$ и $\omega_{3} / \omega_{1}$, что демонстрирует общую связь между интегралами и рядами.

Произвольная эллиптическая функция $h(n)$ порядка $s+1$ с периодами $\omega_{2} / \omega_{1}$ и $\omega_{3} / \omega_{1}$ имеет вид

$$
h(n)=y \prod_{k=1}^{s+1} \frac{\theta\left(t_{k} q^{n} ; p\right)}{\theta\left(w_{k} q^{u} ; p\right)},
$$

где $\prod_{k=1}^{s+1} t_{k}=\prod_{k=1}^{s+1} w_{k}$. Определим эллиптический символ Похгаммера $\theta(t)_{n}$ как решение рекуррентного соотношения $c_{n+1}=\theta\left(t q^{n} ; p\right) c_{n}$ с начальным условием $c_{0}=1$ :

$$
\theta(t)_{n}= \begin{cases}\prod_{j=0}^{n-1} \theta\left(t q^{j} ; p\right), & n>0 \\ \prod_{j=1}^{-n} \frac{1}{\theta\left(t q^{-j} ; p\right)}, & n<0\end{cases}
$$


Тогда легко вывести явный вид формального двустороннего эллиптического гипергеометрического ряда

$$
{ }_{s+1} G_{s+1}\left(\begin{array}{c}
t_{1}, \ldots, t_{s+1} \\
w_{1}, \ldots, w_{s+1}
\end{array} ; q, p ; y\right)=\sum_{n \in \mathbb{Z}} \prod_{k=1}^{s+1} \frac{\theta\left(t_{k}\right)_{n}}{\theta\left(w_{k}\right)_{n}} y^{n}
$$

с нормировкой нулевого коэффициента ряда $c_{0}=1$. Выбрав $w_{s+1}=q$ и заменив $t_{s+1} \rightarrow t_{0}$, получаем односторонний ряд

$$
{ }_{s+1} E_{s}\left(\begin{array}{c}
t_{0}, t_{1}, \ldots, t_{s} \\
w_{1}, \ldots, w_{s}
\end{array} ; q, p ; y\right)=\sum_{n=0}^{\infty} \frac{\theta\left(t_{0}, t_{1}, \ldots, t_{s}\right)_{n}}{\theta\left(q, w_{1}, \ldots, w_{s}\right)_{n}} y^{n} .
$$

Для фиксированных $t_{j}$ и $w_{j}$ в пределе $p \rightarrow 0$ имеем $\theta(t)_{n} \rightarrow(t ; q)_{n}=(1-t) \times$ $(1-q t) \cdots\left(1-q^{n-1} t\right)$, и функция ${ }_{s+1} E_{s}$ переходит в $q$-гипергеометрический ряд [3]

$$
{ }_{s+1} \varphi_{s}\left(\begin{array}{c}
t_{0}, t_{1}, \ldots, t_{s} \\
w_{1}, \ldots, w_{s}
\end{array} ; q ; y\right)=\sum_{n=0}^{\infty} \frac{\left(t_{0} ; q\right)_{n} \cdots\left(t_{s} ; q\right)_{n}}{(q ; q)_{n}\left(w_{1} ; q\right)_{n} \cdots\left(w_{s} ; q\right)_{n}} y^{n}
$$

с условием $\prod_{j=0}^{s} t_{j}=q \prod_{j=1}^{s} w_{j}$. Параметризуя $t_{j}=q^{u_{j}}$ и $w_{j}=q^{v_{j}}$, в пределе $q \rightarrow 1$ получаем ряд

$$
{ }_{s+1} F_{s}\left(\begin{array}{c}
u_{0}, u_{1}, \ldots, u_{s} \\
v_{1}, \ldots, v_{s}
\end{array} ; y\right)=\sum_{n=0}^{\infty} \frac{\left(u_{0}\right)_{n} \cdots\left(u_{s}\right)_{n}}{n !\left(v_{1}\right)_{n} \cdots\left(v_{s}\right)_{n}} y^{n},
$$

где $(a)_{n}=a(a+1) \cdots(a+n-1)$ обозначает обычный символ Похгаммера и $u_{0}+\cdots+u_{s}=1+v_{1}+\cdots+v_{s}$. Последнее ограничение на параметры несущественно, так как оно уже отсутствует для ${ }_{s} F_{s-1}$-функции, полученной предельным переходом $u_{s} \rightarrow \infty$.

Исследование условий сходимости бесконечных рядов (4.11) представляет собой серьезную задачу и до настоящего времени она не решена полностью. Поэтому при использовании $E$-рядов обычно подразумевается, что они обрываются благодаря условию $t_{k}=q^{-N} p^{M}$ для некоторого $k$, где $N=0,1,2, \ldots$ и $M \in \mathbb{Z}$. Отметим, что формальный ${ }_{2} E_{1}$-ряд не является естественным эллиптическим обобщением гипергеометрической функции Гаусса, так как у него нет естественных аналогов многих важных свойств ${ }_{2} F_{1}$-функции.

При выполнении ограничений на параметры $t_{0} q=t_{1} w_{1}=\cdots=t_{s} w_{s}$ ряды (4.11) называются вполне уравновешенными. Для них условие балансировки принимает вид $t_{1} \cdots t_{s}= \pm q^{(s+1) / 2} t_{0}^{(s-1) / 2}$, а функции $h(n)$ и $_{s+1} E_{s}$ становятся инвариантными относительно преобразований $t_{j} \rightarrow p t_{j}, j=1, \ldots, s-1$, и $t_{0} \rightarrow p^{2} t_{0}$ (при этом $t_{s}$ считается зависимым параметром). Так же, как и в случае интегралов, при нечетных $s$ и выборе знака “+" в условии балансировки возникает дополнительная симметрия - функции $h(n)$ и ${ }_{s+1} E_{s}$ становятся инвариантными относительно преобразования $t_{0} \rightarrow p t_{0}$ (с компенсирующим преобразованием $\left.t_{s} \rightarrow p^{(s-1) / 2} t_{s}\right)$. Таким образом, $h(n)$ становится эллиптической функцией всех свободных параметров $\log t_{j}, j=0,1, \ldots, s-1$, с одинаковыми периодами, т. е. появляется некоторая "полная эллиптичность" [10], [31]. 
Следующее структурное ограничение, которое необходимо для построения наиболее интересных примеров рядов, выглядит так:

$$
t_{s-3}=q \sqrt{t_{0}}, \quad t_{s-2}=-q \sqrt{t_{0}}, \quad t_{s-1}=q \sqrt{\frac{t_{0}}{p}}, \quad t_{s}=-q \sqrt{p t_{0}}
$$

и называется условием совершенной уравновешенности (оно связано с удвоением аргумента тэта-функций). Ввиду важности совершенно уравновешенных эллиптических гипергеометрических рядов для них имеются специальные обозначения [52]:

$$
\begin{aligned}
& { }_{s+1} V_{s}\left(t_{0} ; t_{1}, \ldots, t_{s-4} ; q, p ; y\right)=\sum_{n=0}^{\infty} \frac{\theta\left(t_{0} q^{2 n} ; p\right)}{\theta\left(t_{0} ; p\right)} \prod_{m=0}^{s-4} \frac{\theta\left(t_{m}\right)_{n}}{\theta\left(q t_{0} t_{m}^{-1}\right)_{n}}(q y)^{n}
\end{aligned}
$$

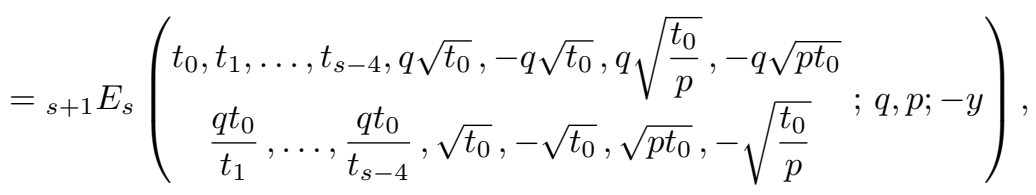

где $\prod_{k=1}^{s-4} t_{k}= \pm t_{0}^{(s-5) / 2} q^{(s-7) / 2}$ (в этом условии балансировки для нечетных $s$ выбор знака "+" считается каноническим). Выбор $t_{j}= \pm \sqrt{q t_{0}}$ устраняет этот параметр и редуцирует индексы ряда на 1 (при этом может измениться знак в условии балансировки). Для достаточно больших $s$ можно подобрать четыре параметра так, что совершенно уравновешенная часть в коэффициентах ряда сократится и он снова станет только вполне уравновешенным рядом. При $y=1$ этот аргумент опускается в обозначениях ${ }_{s+1} V_{s}$-рядов. В этой схеме формула Френкеля-Тураева (3.8) дает замкнутое выражение для обрывающегося ${ }_{10} V_{9}\left(t_{0} ; t_{1}, \ldots, t_{5} ; q, p\right)$-ряда.

Для рассмотрения модулярных преобразований необходимо перейти к параметризации $t_{k}=q^{u_{k}}, w_{k}=q^{v_{k}}, q=e^{2 \pi i \sigma}, p=e^{2 \pi i \tau}$ и заменить $\theta\left(q^{a} ; p\right)$-функции на "эллиптические числа" $[a]=\theta_{1}(\sigma a \mid \tau)$. Предположим, что параметр $y$ не зависит от $\tau$. Тогда с помощью формулы (А.3) (с. 64) нетрудно проверить, что функции $h(n)$ и соответственно ${ }_{s+1} E_{s}$ будут модулярно инвариантными при условии $\sum_{k=0}^{s} u_{k}^{2}=1+\sum_{k=1}^{s} v_{k}^{2}$, определенном по модулю $2 \tau / \sigma^{2}$. Для полноты описания приведем явное выражение для наиболее интересного ${ }_{s+1} V_{s}$-ряда с нечетным $s$ в этих обозначениях:

$$
{ }_{2 m} V_{2 m-1}\left(q^{u_{0}} ; q^{u_{1}}, \ldots, q^{u_{2 m-5}} ; q, p ; y\right)=\sum_{n=0}^{\infty} \frac{\left[2 n+u_{0}\right]}{\left[u_{0}\right]} \prod_{k=0}^{2 m-5} \frac{\left[u_{k}\right]_{n}}{\left[1+u_{0}-u_{k}\right]_{n}} y^{n}
$$

где $[a]_{n}=[a][a+1] \cdots[a+n-1]$, а условие балансировки имеет вид $\sum_{k=1}^{2 m-5} u_{k}=$ $m-4+(m-3) u_{0}$. Этот ряд автоматически модулярно инвариантен. В [3] для аддитивной системы обозначений рядов были предложены ${ }_{s+1} e_{s^{-}}$и $s_{s+1} v_{s}$-символы, но мы не будем ими пользоваться в данной работе.

Важность условия балансировки для обычных и $q$-гипергеометрических функций была известна давно, так как при его наличии появляются некоторые дополнительные тождества [3]. То же самое относится к понятиям вполне 
уравновешенности и совершенной уравновешенности. Появление соответствующих ограничений на параметры выглядело, однако, достаточно произвольным, и не был ясен их глубинный смысл. Эллиптические гипергеометрические функции проясняют происхождение этих старых концепций. Именно, условие балансировки связано с условием двоякой периодичности основной эллиптической функции, использованной при построении рядов или интегралов. Условие вполне уравновешенности связано с условием эллиптичности по всем параметрам, определяющим дивизор этой эллиптической функции. А условие совершенной уравновешенности привязано к правилу удвоения аргумента тэта-функций. Строго говоря, эти понятия на самом деле последовательно определены только на эллиптическом уровне. Действительно, существуют предельные переходы от эллиптических гипергеометрических тождеств, использующих ${ }_{s+1} V_{s}$-ряды, к $q$-гипергеометрическим соотношениям, в результате которых возникают базисные $s+1 \varphi_{s}$-функции, не обладающие каким-либо из упомянутых свойств [35], [52].

Описанное выше вырождение ${ }_{s+1} E_{s}$-рядов при $p \rightarrow 0$ приводит к ограничению на параметры для $s+1 \varphi_{s}$-рядов, похожему на старое условие $q$-балансировки [1], [3], но не совпадающему с ним. Предел $p \rightarrow 0$ для ${ }_{s+1} V_{s}$-рядов с фиксированными параметрами приводит к совершенно уравновешенным сбалансированным ${ }_{s-1} \varphi_{s-2}$-рядам, имеющим свое обозначение [3]:

$$
\begin{aligned}
& \lim _{p \rightarrow 0} s+1 V_{s}\left(t_{0} ; t_{1}, \ldots, t_{s-4} ; q, p ; z\right)=\sum_{n=0}^{\infty} \frac{1-t_{0} q^{2 n}}{1-t_{0}} \prod_{k=0}^{s-4} \frac{\left(t_{k} ; q\right)_{n}}{\left(q t_{0} / t_{k} ; q\right)_{n}}(q z)^{n} \\
& ={ }_{s-1} W_{s-2}\left(t_{0} ; t_{1}, \ldots, t_{s-4} ; q, q z\right) \text {. }
\end{aligned}
$$

Замечательным образом, условие балансировки для ${ }_{s+1} V_{s}$-рядов совпадает в этом случае с обычным условием балансировки для ${ }_{s-1} W_{s-2}$-рядов [3], [10]. Возможность фиксации знака в условии балансировки для нечетных $s$ из требования наличия дополнительной симметрии усиливает важность “эллиптической” точки зрения на функции гипергеометрического типа и указывает на неразрывную связь этих двух классов функций.

В заключение этого раздела упомянем, что квадратичные преобразования для ${ }_{s+1} V_{s}$-рядов и связанные формулы суммирования рассмотрены в [49], [52]-[54]. Другие специфические эллиптические гипергеометрические ряды исследовались в работах [55], [56]. Интересное приложение ${ }_{s+1} E_{s}$-рядов с нетривиальным степенным параметром $y$ появилось недавно в [57].

\section{5. Эллиптический аналог гипергеометрической функции Гаусса}

5.1. Определение $V$-функции и связь с системой корней $E_{7}$. Интегральное представление Эйлера для ${ }_{2} F_{1}(a, b ; c ; x)$-функции отличается от бетаинтеграла тем, что содержит в подынтегральном выражении дополнительный член, зависящий от двух новых параметров [1]. Эллиптический аналог гипергеометрической функции Гаусса, который мы будем обозначать символом 
$V\left(t_{1}, \ldots, t_{8} ; p, q\right)$, также является двухпараметрическим расширением эллиптического бета-интеграла [31]:

$$
V\left(t_{1}, \ldots, t_{8} ; p, q\right)=\kappa \int_{\mathbb{T}} \frac{\prod_{j=1}^{8} \Gamma\left(t_{j} z^{ \pm 1} ; p, q\right)}{\Gamma\left(z^{ \pm 2} ; p, q\right)} \frac{d z}{z},
$$

где восемь параметров $t_{1}, \ldots, t_{8} \in \mathbb{C}$ и две базисные переменные $p, q \in \mathbb{C}$ удовлетворяют ограничениям $\left|t_{j}\right|,|p|,|q|<1$ и условию балансировки $\prod_{j=1}^{8} t_{j}=p^{2} q^{2}$. Для других значений параметров $t_{j} V$-функция определяется аналитическим продолжением интеграла (5.1). Это продолжение строится заменой контура интегрирования $\mathbb{T}$ на контур $C$, отделяющий последовательности полюсов подынтегрального выражения $z=t_{j} p^{a} p^{b}, a, b=0,1,2, \ldots$, сходящихся к нулю, от полюсов $z=t_{j}^{-1} p^{-a} p^{-b}$, уходящих на бесконечность; это уже не подразумевает ограничений $\left|t_{j}\right|<1$. Стягивая контур $C$, можно представить результирующую функцию как интеграл по $\mathbb{T}$ и сумму вычетов полюсов, пересеченных контуром при этой деформации.

Пусть некоторая пара параметров удовлетворяет условию $t_{j} t_{k}=p q$. Тогда $V$-функция редуцируется к эллиптическому бета-интегралу (3.1), что следует из формулы отражения для эллиптической гамма-функции $\Gamma(z ; p, q)$.

Рассмотрим симметрии $V$-функции. Очевидно, что она инвариантна относительно перестановки $p$ и $q$ и $S_{8}$-группы перестановок $t_{j}$, связанной с корневой системой $A_{7}$. Оказывается, что существует преобразование, расширяющее $S_{8}$ до группы Вейля для исключительной системы корней $E_{7}$. Оно находится с помощью двойного интеграла

$$
\kappa \int_{\mathbb{T}^{2}} \frac{\prod_{j=1}^{4} \Gamma\left(a_{j} z^{ \pm 1}, b_{j} w^{ \pm 1} ; p, q\right) \Gamma\left(c z^{ \pm 1} w^{ \pm 1} ; p, q\right)}{\Gamma\left(z^{ \pm 2}, w^{ \pm 2} ; p, q\right)} \frac{d z}{z} \frac{d w}{w}
$$

где $a_{j}, b_{j}, c \in \mathbb{C},\left|a_{j}\right|,\left|b_{j}\right|,|c|<1$ и $c^{2} \prod_{j=1}^{4} a_{j}=c^{2} \prod_{j=1}^{4} b_{j}=p q$. Вычисление интегралов по $z$ или по $w$ в различном порядке с помощью формулы (3.1) приводит к фундаментально важному соотношению [11]

$$
V(\underline{t})=\prod_{1 \leqslant j<k \leqslant 4} \Gamma\left(t_{j} t_{k}, t_{j+4} t_{k+4} ; p, q\right) V(\underline{s})
$$

где $V(\underline{t})=V\left(t_{1}, \ldots, t_{8} ; p, q\right)$ и

$$
\left\{\begin{array}{ll}
s_{j}=\varepsilon t_{j}, & j=1,2,3,4 \\
s_{j}=\varepsilon^{-1} t_{j}, & j=5,6,7,8
\end{array} ; \quad \varepsilon=\sqrt{\frac{p q}{t_{1} t_{2} t_{3} t_{4}}}=\sqrt{\frac{t_{5} t_{6} t_{7} t_{8}}{p q}} \quad \text { и } \quad\left|t_{j}\right|,\left|s_{j}\right|<1 .\right.
$$

Повторное преобразование (5.2) с параметрами $s_{3,4,5,6}$, играющими ту же роль, что и $t_{1,2,3,4}$, и последующая перестановка параметров $t_{3}, t_{4}$ с $t_{5}, t_{6}$ приводят к равенству

$$
V(\underline{t})=\prod_{j, k=1}^{4} \Gamma\left(t_{j} t_{k+4} ; p, q\right) V\left(\frac{T^{1 / 2}}{t_{1}}, \ldots, \frac{T^{1 / 2}}{t_{4}}, \frac{U^{1 / 2}}{t_{5}}, \ldots, \frac{U^{1 / 2}}{t_{8}}\right),
$$


где $T=t_{1} t_{2} t_{3} t_{4}, U=t_{5} t_{6} t_{7} t_{8}$ и $|T|^{1 / 2}<\left|t_{j}\right|<1,|U|^{1 / 2}<\left|t_{j+4}\right|<1, j=1,2,3,4$. Приравнивая правые части равенств (5.2) и (5.3) и выражая параметры $t_{j}$ через $s_{j}$, получаем третье преобразование

$$
V(\underline{s})=\prod_{1 \leqslant j<k \leqslant 8} \Gamma\left(s_{j} s_{k} ; p, q\right) V\left(\frac{\sqrt{p q}}{s_{1}}, \ldots, \frac{\sqrt{p q}}{s_{8}}\right),
$$

где $|p q|^{1 / 2}<\left|s_{j}\right|<1$ для всех $j$.

Свяжем параметры функции $V(\underline{t})$ с координатами евклидова пространства $x_{j} \in \mathbb{R}^{8}$ соотношениями $t_{j}=e^{2 \pi i x_{j}}(p q)^{1 / 4}$. Обозначим через $\langle x, y\rangle$ скалярное произведение в $\mathbb{R}^{8}$ и через $e_{i}$ - какой-либо ортонормированный базис, удовлетворяющий условию $\left\langle e_{i}, e_{j}\right\rangle=\delta_{i j}$. Корневая система $A_{7}$ состоит из векторов $v=\left\{e_{i}-e_{j}, i \neq j\right\}$, а ее группа Вейля $S_{8}-$ из отражений $x \rightarrow S_{v}(x)=x-$ $2 v\langle v, x\rangle /\langle v, v\rangle$, действующих в гиперплоскости, ортогональной вектору $\sum_{i=1}^{8} e_{i}$. При этом координаты векторов $x=\sum_{i=1}^{8} x_{i} e_{i}$ удовлетворяют соотношению $\sum_{i=1}^{8} x_{i}=0$, что обеспечивается условием балансировки.

Преобразование координат в $(5.2)$ соответствует отражению $S_{v}(x)$ по отношению к вектору $v=\left(\sum_{i=5}^{8} e_{i}-\sum_{i=1}^{4} e_{i}\right) / 2$ длины $\langle v, v\rangle=2$. Оно расширяет группу $S_{8}$ до группы Вейля для исключительной системы корней $E_{7}$. Другое доказательство равенств (5.2)-(5.4) дано Рэйнсом в [34], где впервые указана их связь с $E_{7}$-группой.

В [11] показано, что $V$-функция сводится к произведению двух ${ }_{12} V_{11}$-рядов при специальных дискретных значениях одного из параметров. Обозначим $A=t_{0} \cdots t_{4}$; тогда

$$
\begin{aligned}
V\left(t_{0}, \ldots, t_{5}, t_{5}^{-1} p^{n+1} q^{m+1}, A^{-1} p^{1-n} q^{1-m} ; p, q\right) \\
=\frac{\prod_{0 \leqslant j<k \leqslant 4} \Gamma\left(t_{j} t_{k} ; p, q\right)}{\prod_{j=0}^{4} \Gamma\left(t_{j}^{-1} A ; p, q\right)} \frac{\Gamma\left(p^{n+1} q^{m+1} t_{5}^{-1} t_{0}^{ \pm 1}, t_{5} t_{0}^{ \pm 1}, A t_{0}^{ \pm 1} ; p, q\right)}{\Gamma\left(p^{n} q^{m} A t_{0}^{ \pm 1} ; p, q\right)} \\
\quad \times_{12} V_{11}\left(\frac{A t_{0}}{q} ; \frac{A t_{5}}{q}, t_{0} t_{1}, t_{0} t_{2}, t_{0} t_{3}, t_{0} t_{4}, q^{-m}, \frac{A q^{m}}{t_{5}} ; q, p\right) \\
\quad{ }_{12} V_{11}\left(\frac{A t_{0}}{p} ; \frac{A t_{5}}{p}, t_{0} t_{1}, t_{0} t_{2}, t_{0} t_{3}, t_{0} t_{4}, p^{-n}, \frac{A p^{n}}{t_{5}} ; p, q\right),
\end{aligned}
$$

где контур интегрирования в определении $V$-функции выбран так, чтобы разделить последовательности полюсов подынтегральной функции, сходящиеся к нулю и уходящие на бесконечность. При $m=0$ получаем интегральное представление для отдельного обрывающегося ${ }_{12} V_{11}$-ряда. Поскольку левая часть $(5.5)$ симметрична по $t_{0}, \ldots, t_{4}$, это должно быть верно и для правой части, что выполняется благодаря преобразованию симметрии для рядов, которое будет рассмотрено ниже.

5.2. Эллиптическое гипергеометрическое уравнение. Формула сложения для тэта-функций (А.5), записанная в виде

$$
t_{8} \theta\left(t_{7} t_{8}^{ \pm 1}, t_{6} z^{ \pm 1} ; p\right)+t_{6} \theta\left(t_{8} t_{6}^{ \pm 1}, t_{7} z^{ \pm 1} ; p\right)+t_{7} \theta\left(t_{6} t_{7}^{ \pm 1}, t_{8} z^{ \pm 1} ; p\right)=0
$$


приводит к формуле связи

$$
\frac{t_{6} V\left(q t_{6}\right)}{\theta\left(t_{6} t_{7}^{ \pm 1}, t_{6} t_{8}^{ \pm 1} ; p\right)}+\frac{t_{7} V\left(q t_{7}\right)}{\theta\left(t_{7} t_{6}^{ \pm 1}, t_{7} t_{8}^{ \pm 1} ; p\right)}+\frac{t_{8} V\left(q t_{8}\right)}{\theta\left(t_{8} t_{6}^{ \pm 1}, t_{8} t_{7}^{ \pm 1} ; p\right)}=0
$$

где $V\left(q t_{j}\right)$ обозначает $V$-функции с параметром $t_{j}$, замененным на $q t_{j}$ (это приводит к условию балансировки $\left.\prod_{j=1}^{8} t_{j}=p^{2} q\right)$. Действительно, легко проверить выполнение такого же равенства для ядра $V$-функции, после интегрирования которого по $z$ и получается (5.6).

Подстановка преобразования (5.4) в (5.6) дает

$$
\begin{aligned}
& \frac{\prod_{j=1}^{5} \theta\left(t_{6} t_{j} / q ; p\right)}{t_{6} \theta\left(t_{7} / t_{6}, t_{8} / t_{6} ; p\right)} V\left(q^{-1} t_{6}\right)+\frac{\prod_{j=1}^{5} \theta\left(t_{7} t_{j} / q ; p\right)}{t_{7} \theta\left(t_{6} / t_{7}, t_{8} / t_{7} ; p\right)} V\left(q^{-1} t_{7}\right) \\
& \quad+\frac{\prod_{j=1}^{5} \theta\left(t_{8} t_{j} / q ; p\right)}{t_{8} \theta\left(t_{6} / t_{8}, t_{7} / t_{8} ; p\right)} V\left(q^{-1} t_{8}\right)=0,
\end{aligned}
$$

где $\prod_{j=1}^{8} t_{j}=p^{2} q^{3}$.

Рассмотрим три уравнения, получающиеся заменами $t_{8} \rightarrow q t_{8}$ в (5.7) и $t_{6} \rightarrow q^{-1} t_{6}$ или $t_{7} \rightarrow q^{-1} t_{7}$ в (5.6). Исключая из них функции $V\left(q^{-1} t_{6}, q t_{8}\right)$ и $V\left(q^{-1} t_{7}, q t_{8}\right)$, мы получаем эллиптическое гипергеометрическое уравнение [31], [58]:

$$
\begin{aligned}
& \mathscr{A}\left(t_{1}, \ldots, t_{6}, t_{7}, t_{8}, q ; p\right)\left(U\left(q t_{6}, q^{-1} t_{7}\right)-U(\underline{t})\right) \\
& \quad+\mathscr{A}\left(t_{1}, \ldots, t_{7}, t_{6}, t_{8}, q ; p\right)\left(U\left(q^{-1} t_{6}, q t_{7}\right)-U(\underline{t})\right)+U(\underline{t})=0,
\end{aligned}
$$

где

$$
\mathscr{A}\left(t_{1}, \ldots, t_{8}, q ; p\right):=\frac{\theta\left(t_{6} / q t_{8}, t_{8} t_{6}, t_{8} / t_{6} ; p\right)}{\theta\left(t_{6} / t_{7}, t_{7} / q t_{6}, t_{6} t_{7} / q ; p\right)} \prod_{k=1}^{5} \frac{\theta\left(t_{7} t_{k} / q ; p\right)}{\theta\left(t_{8} t_{k} ; p\right)}
$$

и

$$
U(\underline{t}):=U(\underline{t} ; p, q)=\frac{V(\underline{t} ; p, q)}{\prod_{k=6}^{7} \Gamma\left(t_{k} t_{8}^{ \pm 1} ; p, q\right)} .
$$

Потенциал $\mathscr{A}\left(t_{1}, \ldots, t_{8}, q ; p\right)$ является эллиптической функцией всех своих параметров, т. е. он не меняется при заменах $t_{j} \rightarrow p t_{j}, j=1, \ldots, 7$, если считать $t_{8}$ параметром, зависящим от остальных через условие балансировки. Ввиду симметрии $U$-функции по $p$ и $q$, она удовлетворяет еще одному эллиптическому гипергеометрическому уравнению, получающемуся из (5.8) перестановкой $p$ и $q$. Замена переменных приводит функцию $\mathscr{A}\left(t_{1}, \ldots, t_{8}, q ; p\right)$ к полностью $S_{8}$-симметричному виду (4.6) с произвольными $u_{1}, \ldots, u_{8}$ и $\mu=1$ (замечание Д. Цагира автору). Однако само эллиптическое гипергеометрическое уравнение обладает максимально $S_{6}$-симметрией [31], [58].

Обозначим $t_{6}=c x, t_{7}=c / x$ и перейдем к новому набору параметров

$$
\varepsilon_{k}=\frac{q}{c t_{k}}, \quad k=1, \ldots, 5, \quad \varepsilon_{8}=\frac{c}{t_{8}}, \quad \varepsilon_{7}=\frac{\varepsilon_{8}}{q} .
$$

Выберем параметр $\varepsilon_{6}$ исходя из требования выполнения условия балансировки в виде $\prod_{k=1}^{8} \varepsilon_{k}=p^{2} q^{2}$, что дает $c=\sqrt{\varepsilon_{6} \varepsilon_{8}} / p^{2}$. Теперь после замены $U(\underline{t})$ 
на неизвестную функцию $f(x)$ в (5.8) мы можем переписать это эллиптическое гипергеометрическое уравнение в виде $q$-разностного уравнения второго порядка:

$$
\begin{gathered}
A(x)(f(q x)-f(x))+A\left(x^{-1}\right)\left(f\left(q^{-1} x\right)-f(x)\right)+\nu f(x)=0, \\
A(x)=\frac{\prod_{k=1}^{8} \theta\left(\varepsilon_{k} x ; p\right)}{\theta\left(x^{2}, q x^{2} ; p\right)}, \quad \nu=\prod_{k=1}^{6} \theta\left(\frac{\varepsilon_{k} \varepsilon_{8}}{q} ; p\right),
\end{gathered}
$$

в котором явно видна $S_{6}$-группа симметрий по параметрам. Одно функциональное решение этого уравнения у нас уже есть:

$$
f_{1}(x ; \underline{\varepsilon} ; p, q)=\frac{V\left(q /\left(c \varepsilon_{1}\right), \ldots, q /\left(c \varepsilon_{5}\right), c x, c / x, c / \varepsilon_{8} ; p, q\right)}{\Gamma\left(c^{2} x^{ \pm 1} / \varepsilon_{8}, x^{ \pm 1} \varepsilon_{8} ; p, q\right)} .
$$

Для построения других линейно независимых решений можно использовать симметрии уравнения (5.10), не являющимися симметриями функции (5.12). Например, второе решение может быть получено умножением одного из параметров $\varepsilon_{1}, \ldots, \varepsilon_{5}$ или $x$ на степени $p$ или перестановками $\varepsilon_{1}, \ldots, \varepsilon_{5}$ с $\varepsilon_{6}$.

Поскольку функция $\mathscr{A}(\underline{t}, q ; p)$ в уравнении $(5.8)$ не меняется при заменах $t_{6} \rightarrow p^{-1} t_{6}, t_{7} \rightarrow p t_{7}$, то функция $U\left(p^{-1} t_{6}, p t_{7}\right)$ также является решением этого уравнения. В работе [59] вычислен казоратиан (дискретный вронскиан) этих двух решений. Умножим уравнение (5.8) на $U\left(p^{-1} t_{6}, p t_{7}\right)$, а уравнение для $U\left(p^{-1} t_{6}, p t_{7}\right)$ на $U(\underline{t})$ и вычтем одно получающееся соотношение из другого. В результате возникает равенство

$$
\mathscr{A}\left(t_{1}, \ldots, t_{6}, t_{7}, t_{8}, q ; p\right) D\left(p^{-1} t_{6}, q^{-1} t_{7}\right)=\mathscr{A}\left(t_{1}, \ldots, t_{7}, t_{6}, t_{8}, q ; p\right) D\left(p^{-1} q^{-1} t_{6}, t_{7}\right),
$$

где $D$ обозначает казоратиан

$$
D\left(t_{6}, t_{7}\right)=U\left(p q t_{6}, t_{7}\right) U\left(t_{6}, p q t_{7}\right)-U\left(q t_{6}, p t_{7}\right) U\left(p t_{6}, q t_{7}\right) .
$$

После подстановок $t_{6} \rightarrow p t_{6}, t_{7} \rightarrow q t_{7}$ условие балансировки принимает вид $t_{6} t_{7}=p q /\left(t_{1} \cdots t_{5} t_{8}\right)$, и (5.13) можно рассматривать как $q$-разностное уравнение первого порядка по $t_{7}$. Оно легко решается, и его решение определено с точностью до умножения на произвольную $q$-периодическую функцию $\varphi\left(q t_{7}\right)=\varphi\left(t_{7}\right)$.

Повторяя эту же процедуру с уравнениями, получающимися после перестановки параметров $p$ и $q$, находим $\varphi\left(p t_{7}\right)=\varphi\left(t_{7}\right)$, так что $\varphi\left(t_{7}\right)$ не зависит от $t_{7}$. Дальнейшее исследование структуры вычетов полюсов $V$-функций в $D\left(t_{6}, t_{7}\right)$, пересекаемых контуром интегрирования в пределе $t_{7} \rightarrow t_{8}^{-1}$, полностью фиксирует вид $\varphi$ и приводит к тождеству [59]

$$
V\left(p q t_{6}, t_{7}\right) V\left(t_{6}, p q t_{7}\right)-t_{6}^{-2} t_{7}^{-2} V\left(q t_{6}, p t_{7}\right) V\left(p t_{6}, q t_{7}\right)=\frac{\prod_{1 \leqslant j<k \leqslant 8} \Gamma\left(t_{j} t_{k} ; p, q\right)}{\Gamma\left(t_{6}^{ \pm 1} t_{7}^{ \pm 1} ; p, q\right)},
$$

в котором $t_{6}$ и $t_{7}$ могут быть заменены на любую другую пару параметров.

Описанные решения эллиптического гипергеометрического уравнения существуют при $|q|<1$, в то время как само уравнение (5.8) не требует такого ограничения. Благодаря симметрии

$$
\mathscr{A}\left(\frac{p^{1 / 2}}{t_{1}}, \ldots, \frac{p^{1 / 2}}{t_{8}}, q ; p\right)=\mathscr{A}\left(t_{1}, \ldots, t_{8}, q^{-1} ; p\right),
$$


преобразование $t_{j} \rightarrow p^{1 / 2} / t_{j}, j=1, \ldots, 8$, приводит к изменению базисной переменной $q \rightarrow q^{-1}$ в (5.8). Это дает следующее решение эллиптического гипергеометрического уравнения при $|q|>1$ (см. [59]):

$$
U(\underline{t} ; p, q)=\frac{V\left(p^{1 / 2} / t_{1}, \ldots, p^{1 / 2} / t_{8} ; p, q^{-1}\right)}{\prod_{k=6}^{7} \Gamma\left(p /\left(t_{k} t_{8}\right), t_{8} / t_{k} ; p, q^{-1}\right)} .
$$

Для построения решений уравнения (5.8) (или (5.10)) при $|q|=1$ можно воспользоваться параметризацией базисных переменных (2.6), (2.7), сделать подстановки $t_{k}=e^{2 \pi i g_{k} / \omega_{2}}$ и повторить всю схему рассуждений, приведенную выше, с заменой $\Gamma(z ; p, q)$ на модифицированную эллиптическую гамма-функцию $G(u ; \boldsymbol{\omega})$. Так же, как и в случае с модифицированным эллиптическим бета-интегралом, мы опять получим $V$-функцию, но с другой параметризацией. Эта процедура оказывается эквивалентной применению модулярного преобразования $\left(\omega_{2}, \omega_{3}\right) \rightarrow\left(-\omega_{3}, \omega_{2}\right)$ к решениям, описанным выше. На самом деле, потенциал $\mathscr{A}\left(e^{2 \pi i g_{1} / \omega_{2}}, \ldots, e^{2 \pi i g_{8} / \omega_{2}}, q ; p\right)$ инвариантен относительно этого преобразования, а $U\left(t_{1}, \ldots, t_{8} ; p, q\right)$ переходит в $U\left(e^{-2 \pi i g_{1} / \omega_{3}}, \ldots, e^{-2 \pi i g_{8} / \omega_{3}} ; \tilde{p}, \tilde{r}\right)$. Последняя функция дает таким образом новое решение эллиптического гипергеометрического уравнения, корректно определенное при $|q|=1$. Очевидно, что эта функция удовлетворяет также и партнеру уравнения (5.8), получающемуся из него перестановкой $\omega_{1}$ и $\omega_{2}$. Пример аналогичной ситуации с двумя уравнениями для одной функции на $q$-гипергеометрическом уровне приведен в [60].

Различные формальные вырождения $V$-функции к $q$-гипергеометрическим интегралам типа Меллина-Барнса или Эйлера кратко описаны в [31], [58]. Подробный и строгий анализ процедуры вырождения проведен в [28], [61]. Необходимо отметить [58], что эллиптическое гипергеометрическое уравнение появляется как частный случай уравнения на собственные значения для одночастичного гамильтониана квантовой модели, предложенной ван Диеженом [62] и детально изученной Комори и Хиками [63]. Эта модель типа Калоджеро-Сазерленда является обобщением систем Руджинарса [64] и Иноземцева [65].

\section{6. Цепочки преобразований симметрии для функций}

Преобразования симметрии для обычных и $q$-гипергеометрических рядов строятся с помощью цепочек Бэйли [1], [66]. Эта техника была обобщена на эллиптический уровень в [52], [67]. Изложим кратко это обобщение.

По определению две последовательности чисел $\alpha_{n}(a, k)$ и $\beta_{n}(a, k)$ образуют эллиптическую пару Бэйли по отношению к параметрам $a$ и $k$, если

$$
\beta_{n}(a, k)=\sum_{0 \leqslant m \leqslant n} M_{n m}(a, k) \alpha_{m}(a, k),
$$

где

$$
M_{n m}(a, k)=\frac{\theta(k / a)_{n-m} \theta(k)_{n+m}}{\theta(q)_{n-m} \theta(a q)_{n+m}} \frac{\theta\left(a q^{2 m} ; p\right)}{\theta(a ; p)} a^{n-m} .
$$

В матричном виде $\beta(a, k)=M(a, k) \alpha(a, k)$, где $\alpha$ и $\beta$ обозначают столбцы, образованные $\alpha_{n}$ и $\beta_{n}$. 
Определим диагональную матрицу

$$
D_{n m}(a ; b, c)=D_{m}(a ; b, c) \delta_{n m}, \quad D_{m}(a ; b, c)=\frac{\theta(b, c)_{m}}{\theta(a q / b, a q / c)_{m}}\left(\frac{a q}{b c}\right)^{m} .
$$

ТеОРема 4. Пусть $\alpha(a, t)$ u $\beta(a, t)$ образуют эллиптическую пару Бэйли по отношению $\kappa$ параметрам а $u t$. Тогда величины

$$
\begin{gathered}
\alpha^{\prime}(a, k)=D(a ; b, c) \alpha(a, t), \quad \beta^{\prime}(a, k)=K(t, k, b, c) \beta(a, t), \\
K(t, k, b, c):=D\left(k ; \frac{q t}{b}, \frac{q t}{c}\right) M(t, k) D(t ; b, c),
\end{gathered}
$$

где qat $=k b c u b, c-$ два произвольных новых параметра, образуют новую эллиптическую пару Бэйли по отношению $к$ а $и$.

ДокАЗАТЕЛЬСтво. Подстановка (6.4) и соотношения $\beta=M \alpha$ в требуемое равенство $\beta^{\prime}=M \alpha^{\prime}$ приводит к матричному тождеству

$$
M(a, k) D(a ; b, c) M(t, a)=D\left(k ; \frac{q t}{b}, \frac{q t}{c}\right) M(t, k) D(t ; b, c) .
$$

После подстановки явного вида матриц можно увидеть, что оно эквивалентно формуле суммирования Френкеля-Тураева (3.8).

Поскольку $M_{n m}(a, a)=\delta_{n m}$ и $D_{n m}(b c / q ; b, c)=\delta_{n m}$, полагая $t=k$ в $(6.5)$, находим $M(a, k) M(k, a)=1$. Таким образом, обращение матрицы $M$ достигается перестановкой параметров $a$ и $k$ (для случая $p=0$ этот факт был установлен в [68]; более детальное обсуждение подобных обращений матриц дается в [53], [56], [69]). Поэтому $\tilde{\alpha}(a, k)=\beta(k, a)$ и $\tilde{\beta}(a, k)=\alpha(k, a)$ определяют новые пары Бэйли, к которым можно применить преобразования (6.4). Описанные правила составления новых пар Бэйли порождают двоичное дерево тождеств для различных произведений матриц $M$ и $D$, эквивалентных некоторым нетривиальным тождествам для (многократных) эллиптических гипергеометрических рядов.

Из матричного соотношения $M(a, f) M(f, a)=1$ находим простейшие пары Бэйли $\alpha_{n}^{(i)}(a, f)=M_{n i}(f, a)$ и $\beta_{n}^{(i)}(a, f)=\delta_{n i}$. Положим $\alpha^{\prime}(a, t)=D(a ; d, e) \times$ $M(f, a)$ и $\beta^{\prime}(a, t)=K(f, t, d, e)$, где qaf $=t d e$ и подразумевается, что $\alpha^{\prime}$ и $\beta^{\prime}$ являются матрицами, столбцы которых образуют пары Бэйли. Тогда равенство $\beta^{\prime}(a, t)=M(a, t) \alpha^{\prime}(a, t)$ эквивалентно (6.5). Соотношение $\beta^{\prime \prime}(a, k)=M(a, k) \times$ $\alpha^{\prime \prime}(a, k)$ с $\alpha^{\prime \prime}(a, k)=D(a ; b, c) \alpha^{\prime}(a, t)$ и $\beta^{\prime \prime}(a, k)=K(t, k, b, c) \beta^{\prime}(a, t)$, где qat $=$ $k b c$, приводит к тождеству

$$
\begin{aligned}
& { }_{12} V_{11}\left(a ; b, c, d, e, k q^{n}, q^{-n}, a f^{-1} ; q, p\right)=\frac{\theta(q a, k / t, q t / b, q t / c)_{n}}{\theta(k / a, q t, k b / t, k c / t)_{n}} \\
& \times{ }_{12} V_{11}\left(t ; b, c, \frac{t d}{a}, \frac{t e}{a}, k q^{n}, q^{-n}, t f^{-1} ; q, p\right)
\end{aligned}
$$

где $f=k b c d e /(a q)^{2}$. Это соотношение представляет собой эллиптический аналог преобразования Бэйли для обрывающихся 10 $\varphi_{9}$-рядов [3], впервые доказанный в [8] другим способом. Существует также четырехчленное преобразование 
Бэйли для необрывающихся 10 $\varphi_{9}$-рядов [3]. Его эллиптическим обобщением является преобразование $V$-функции (5.8) (записанное в интегральной форме, поскольку его аналог в виде бесконечных рядов не имеет корректного определения).

Интегральные аналоги цепочек Бэйли были открыты в работе [30], где с помощью этой техники построен ряд преобразований симметрии для эллиптических гипергеометрических интегралов. Функции $\alpha(z, t)$ и $\beta(z, t)$ образуют по определению эллиптическую интегральную пару Бэйли по отношению к параметру $t$, если они связаны соотношением

$$
\beta(w, t)=\kappa \int_{\mathbb{T}} \Gamma\left(t w^{ \pm 1} z^{ \pm 1} ; p, q\right) \alpha(z, t) \frac{d z}{z} .
$$

ТЕОРема 5. Пусть $\alpha(z, t)$ u $\beta(z, t)$ образуют эллиптическую интегральную пару Бэйли по отношению $\kappa$ параметру $t,|t|<1$. Возъмем параметры $w, u, s$, удовлетворяющие условиям $w \in \mathbb{T} u|s|,|u|<1,|p q|<\left|t^{2} s^{2} u\right|$. Тогда функиии

$$
\begin{aligned}
& \alpha^{\prime}(w, s t)=\frac{\Gamma\left(t u w^{ \pm 1} ; p, q\right)}{\Gamma\left(t s^{2} u w^{ \pm 1} ; p, q\right)} \alpha(w, t) \\
& \beta^{\prime}(w, s t)=\kappa \frac{\Gamma\left(t^{2} s^{2}, t^{2} s u w^{ \pm 1} ; p, q\right)}{\Gamma\left(s^{2}, t^{2}, s u w^{ \pm 1} ; p, q\right)} \int_{\mathbb{T}} \frac{\Gamma\left(s w^{ \pm 1} x^{ \pm 1}, u x^{ \pm 1} ; p, q\right)}{\Gamma\left(x^{ \pm 2}, t^{2} s^{2} u x^{ \pm 1} ; p, q\right)} \beta(x, t) \frac{d x}{x}
\end{aligned}
$$

определяют новую интегралъную пару Бэйли по отношению $\kappa$ параметру st, а функиии

$$
\begin{aligned}
\alpha^{\prime}(w, t) & =\kappa \frac{\Gamma\left(s^{2} t^{2}, u w^{ \pm 1} ; p, q\right)}{\Gamma\left(s^{2}, t^{2}, w^{ \pm 2}, t^{2} s^{2} u w^{ \pm 1} ; p, q\right)} \int_{\mathbb{T}} \frac{\Gamma\left(t^{2} s u x^{ \pm 1}, s w^{ \pm 1} x^{ \pm 1} p, q\right)}{\Gamma\left(s u x^{ \pm 1} ; p, q\right)} \alpha(x, s t) \frac{d x}{x}, \\
\beta^{\prime}(w, t) & =\frac{\Gamma\left(t u w^{ \pm 1} ; p, q\right)}{\Gamma\left(t s^{2} u w^{ \pm 1} ; p, q\right)} \beta(w, s t)
\end{aligned}
$$

определяют новую интегральную пару Бэйли по отношению $\kappa$ параметру $t$.

Для доказательства первого утверждения достаточно подставить соотношение (6.7) в определение $\beta^{\prime}(w, s t)$, поменять порядок интегрирования и воспользоваться эллиптическим бета-интегралом (3.1). По такой же схеме доказывается и второе утверждение.

В работе [36] показано, что при определенных ограничениях интегральное преобразование $\alpha(x, t) \rightarrow \beta(x, t)$ имеет очень простое обращение. Пусть $p, q, t \in \mathbb{C}$ таковы, что $|p|,|q|<|t|^{2}<1$. Для фиксированного $w \in \mathbb{T}$ обозначим через $C_{w}$ контур внутри кольца $A=\left\{\left.z \in \mathbb{C}|| t|-\epsilon<| z|<| t\right|^{-1}+\epsilon\right\}$ для бесконечно малого положительного $\epsilon$ такого, что точки $t^{-1} w^{ \pm 1}$ лежат внутри $C_{w}$. Пусть $f(z, t)$ - голоморфная функция на $A$, удовлетворяющая $f(z, t)=$ $f\left(z^{-1}, t\right)$. Определим интегральное преобразование

$$
g(w, t)=\kappa \int_{C_{w}} \delta\left(z, w ; t^{-1}\right) f(z, t) \frac{d z}{z},
$$

где

$$
\delta\left(z, w ; t^{-1}\right)=\frac{\Gamma\left(t^{-1} w^{ \pm 1} z^{ \pm 1} ; p, q\right)}{\Gamma\left(t^{2}, z^{ \pm 2} ; p, q\right)} .
$$


Тогда при $|t|<|z|<|t|^{-1}$ верно

$$
f(z, t)=\kappa \int_{\mathbb{T}} \delta(w, z ; t) g(w, t) \frac{d w}{w} .
$$

Это соотношение совпадает по сути с определением эллиптических интегральных пар Бэйли. Можно показать, что указанные выше два способа построения цепочек интегральных пар Бэйли связаны друг с другом приведенным обращением интегрального преобразования (6.8).

С помощью теоремы 5 можно порождать бесконечные последовательности пар Бэйли, стартуя от некоторой начальной пары. Простейшую пару можно построить с помощью эллиптического бета-интеграла (3.1). Каждое новое применение указанных выше подстановок вводит два новых параметра. Равенство (6.7), примененное к возникающим новым парам Бэйли, приводит к бинарному дереву тождеств для многократных эллиптических гипергеометрических интегралов со многими параметрами.

В качестве иллюстрации приведем цепочку нетривиальных соотношений для интегралов

$$
I^{(m)}\left(t_{1}, \ldots, t_{2 m+6}\right)=\kappa \int_{\mathbb{T}} \frac{\prod_{j=1}^{2 m+6} \Gamma\left(t_{j} z^{ \pm 1} ; p, q\right)}{\Gamma\left(z^{ \pm 2} ; p, q\right)} \frac{d z}{z}, \quad \prod_{j=1}^{2 m+6} t_{j}=(p q)^{m+1},
$$

где $\left|t_{j}\right|<1$. С помощью эллиптического бета-интеграла (3.1) легко убедиться в справедливости рекурсии

$$
\begin{aligned}
& I^{(m+1)}\left(t_{1}, \ldots, t_{2 m+8}\right)=\frac{\prod_{2 m+5 \leqslant k<l \leqslant 2 m+8} \Gamma\left(t_{k} t_{l} ; p, q\right)}{\Gamma\left(\rho_{m}^{2} ; p, q\right)} \\
& \quad \times \kappa \int_{\mathbb{T}} \frac{\prod_{k=2 m+5}^{2 m+8} \Gamma\left(\rho_{m}^{-1} t_{k} w^{ \pm 1} ; p, q\right)}{\Gamma\left(w^{ \pm 2} ; p, q\right)} I^{(m)}\left(t_{1}, \ldots, t_{2 m+4}, \rho_{m} w, \rho_{m} w^{-1}\right) \frac{d w}{w},
\end{aligned}
$$

где $\rho_{m}^{2}=\prod_{k=2 m+5}^{2 m+8} t_{k} /(p q)$. Это равенство дает конкретную реализацию пар Бэйли $\alpha \sim I^{(m)}$ и $\beta \sim I^{(m+1)}$ после переобозначения параметров. При $m=0$ подстановка явного выражения для $I^{(0)}(3.1)$ в правую часть (6.11) приводит к тождеству (5.2). Другое важное следствие рекурсии (6.11) рассматривается в следующем разделе. В общем случае равенство (6.11) порождает $m$-кратное интегральное представление для $I^{(m)}$, аналогичное эйлеровскому представлению для ${ }_{m+1} F_{m}$-функций.

\section{7. Биортогональные функции гипергеометрического типа}

7.1. Дискретные биортогональные функции с непрерывной мерой. Обозначим ряд $\mathscr{E}(\mathbf{t}):={ }_{12} V_{11}\left(t_{0}^{2} ; t_{0} t_{1}, \ldots, t_{0} t_{7} ; q, p\right)$ с условиями балансировки $\prod_{m=0}^{7} t_{m}=q^{2}$ и обрыва $t_{0} t_{k}=q^{-n}$ для некоторого $k$. Смежные с $\mathscr{E}(\mathbf{t})$ функции, получающиеся заменой параметров $t_{i}$ и $t_{k}$ на $t_{i} q$ и $t_{k} q^{-1}$, будем обозначать $\mathscr{E}\left(t_{i}^{+}, t_{k}^{-}\right)$. Тогда с помощью формулы сложения для тэта-функций нетрудно 
проверить равенство [9], [46]

$$
\mathscr{E}(\mathbf{t})-\mathscr{E}\left(t_{6}^{-}, t_{7}^{+}\right)=\frac{\theta\left(q t_{0}^{2}, q^{2} t_{0}^{2}, q t_{7} / t_{6}, t_{6} t_{7} / q ; p\right)}{\theta\left(q t_{0} / t_{6}, q^{2} t_{0} / t_{6}, t_{0} / t_{7}, t_{7} / q t_{0} ; p\right)} \prod_{r=1}^{5} \frac{\theta\left(t_{0} t_{r} ; p\right)}{\theta\left(q t_{0} / t_{r} ; p\right)} \mathscr{E}\left(t_{0}^{+}, t_{6}^{-}\right) .
$$

Подстановка эллиптического аналога преобразования Бэйли и его итераций в (7.1) позволяет построить множество других формул подобного типа. Одна из них имеет вид

$$
\begin{gathered}
\frac{\theta\left(t_{0} t_{7} ; p\right)}{\theta\left(t_{6} /\left(q t_{0}\right), t_{6} /\left(q^{2} t_{0}\right), t_{6} / t_{7} ; p\right)} \prod_{r=1}^{5} \theta\left(\frac{t_{r} t_{6}}{q} ; p\right) \mathscr{E}\left(t_{0}^{+}, t_{6}^{-}\right) \\
+\frac{\theta\left(t_{0} t_{6} ; p\right)}{\theta\left(t_{7} /\left(q t_{0}\right), t_{7} /\left(q^{2} t_{0}\right), t_{7} / t_{6} ; p\right)} \prod_{r=1}^{5} \theta\left(\frac{t_{r} t_{7}}{q} ; p\right) \mathscr{E}\left(t_{0}^{+}, t_{7}^{-}\right) \\
=\frac{1}{\theta\left(q t_{0}^{2}, q^{2} t_{0}^{2} ; p\right)} \prod_{r=1}^{5} \theta\left(\frac{q t_{0}}{t_{r}} ; p\right) \mathscr{E}(\mathbf{t}) .
\end{gathered}
$$

Заменим в (7.1) параметр $t_{6}$ на $q t_{6}$, а $t_{7}$ на $t_{7} / q$ и подставим $\mathscr{E}\left(t_{0}^{+}, t_{7}^{-}\right)$из получающегося равенства и $\mathscr{E}\left(t_{0}^{+}, t_{6}^{-}\right)$из $(7.1)$ в $(7.2)$. Это приводит к уравнению

$$
\begin{aligned}
& \frac{\theta\left(t_{0} t_{7}, t_{0} / t_{7}, q t_{0} / t_{7} ; p\right)}{\theta\left(q t_{7} / t_{6}, t_{7} / t_{6} ; p\right)} \prod_{r=1}^{5} \theta\left(\frac{q}{t_{6} t_{r}} ; p\right)\left(\mathscr{E}\left(t_{6}^{-}, t_{7}^{+}\right)-\mathscr{E}(\mathbf{t})\right) \\
& \quad+\frac{\theta\left(t_{0} t_{6}, t_{0} / t_{6}, q t_{0} / t_{6} ; p\right)}{\theta\left(q t_{6} / t_{7}, t_{6} / t_{7} ; p\right)} \prod_{r=1}^{5} \theta\left(\frac{q}{t_{7} t_{r}} ; p\right)\left(\mathscr{E}\left(t_{6}^{+}, t_{7}^{-}\right)-\mathscr{E}(\mathbf{t})\right) \\
& +\theta\left(\frac{q}{t_{6} t_{7}} ; p\right) \prod_{r=1}^{5} \theta\left(t_{0} t_{r} ; p\right) \mathscr{E}(\mathbf{t})=0
\end{aligned}
$$

Эти соотношения аналогичны уравнениям (5.6), (5.7) и (5.8) для $V$-функции и могут быть получены из них анализом вычетов для определенной последовательности полюсов подынтегральной функции. Заменим в (7.3) $\mathscr{E}$-функцию на

$$
R_{n}(x ; q, p)={ }_{12} V_{11}\left(\frac{\varepsilon_{6}}{\varepsilon_{8}} ; \frac{q}{\varepsilon_{1} \varepsilon_{8}}, \frac{q}{\varepsilon_{2} \varepsilon_{8}}, \frac{q}{\varepsilon_{3} \varepsilon_{8}}, q^{-n}, \frac{A q^{n-1}}{\varepsilon_{8}}, \frac{\varepsilon_{6}}{x}, \varepsilon_{6} x ; q, p\right),
$$

где $n=0,1,2, \ldots$ и $A=\varepsilon_{1} \varepsilon_{2} \varepsilon_{3} \varepsilon_{6} \varepsilon_{8}$, и обозначим $q^{-n}=p q /\left(\varepsilon_{4} \varepsilon_{8}\right), A q^{n-1} / \varepsilon_{8}=$ $p q /\left(\varepsilon_{5} \varepsilon_{8}\right)$. Тогда после переобозначения параметров $t_{0}^{2}=\varepsilon_{6} / \varepsilon_{8}, \ldots, t_{0} t_{7}=$ $\varepsilon_{6} x$ мы получим уравнение (5.10) с $\varepsilon_{7}=\varepsilon_{8} / q$ и с дискретными значениями одного из параметров. Таким образом, $R_{n}(x ; q, p)$ определяет частное решение эллиптического гипергеометрического уравнения, обладающее свойством $R_{n}(p x ; q, p)=R_{n}(x ; q, p)$. (Обозначения работы [11], где изучалась эта функция, переходят в наши после замен $t_{0,1,2} \rightarrow \varepsilon_{1,2,3}, t_{3} \rightarrow \varepsilon_{6}, t_{4} \rightarrow \varepsilon_{8}, \mu \rightarrow \varepsilon_{4} \varepsilon_{8} /(p q)$ и $A \mu /\left(q t_{4}\right) \rightarrow p q /\left(\varepsilon_{5} \varepsilon_{8}\right)$.)

Параметры $\varepsilon_{1}, \ldots, \varepsilon_{6}$ входят в (5.10) симметричным образом. В силу условия балансировки $\prod_{k=1}^{8} \varepsilon_{k}=p^{2} q^{2}$ функция $R_{n}(x ; q, p)$ инвариантна относительно замен $\varepsilon_{k} \rightarrow p \varepsilon_{k}, k=1, \ldots, 5$. Это обеспечивает симметричность $(7.4)$ 
по $\varepsilon_{1}, \ldots, \varepsilon_{5}$, и любой из этих параметров можно использовать для обрыва ряда. Перестановка одного из параметров $\varepsilon_{1,2,3,5}$ с $\varepsilon_{6}$ и использование преобразования Бэйли для обрывающихся ${ }_{12} V_{11}$-рядов приводят к $R_{n}(x ; q, p)$ с точностью до некоторого множителя, не зависящего от $x$.

Отождествим теперь параметры в уравнении (7.3) другим способом:

$$
\begin{aligned}
& t_{0}^{2}=\frac{\varepsilon_{6}}{\varepsilon_{8}}, \quad t_{0} t_{1,2,3}=\frac{q}{\varepsilon_{1,2,3} \varepsilon_{8}}, \quad t_{0} t_{4}=\frac{\varepsilon_{6}}{x}, \\
& t_{0} t_{5}=\varepsilon_{6} x, \quad t_{0} t_{6}=q^{-n}, \quad t_{0} t_{7}=\frac{A}{\varepsilon_{8}} q^{n-1} .
\end{aligned}
$$

Это приводит к трехчленному рекуррентному соотношению по индексу $n$ :

$$
\begin{aligned}
& \frac{\theta\left(A q^{n-1} / \varepsilon_{8}, \varepsilon_{6} q^{2-n} / A, \varepsilon_{6} q^{1-n} / A ; p\right)}{\theta\left(A q^{2 n-1} / \varepsilon_{8}, A q^{2 n} / \varepsilon_{8} ; p\right)} \prod_{r=1}^{3} \theta\left(\varepsilon_{r} \varepsilon_{6} q^{n} ; p\right) \theta\left(\frac{q^{n+1} x^{ \pm 1}}{\varepsilon_{8}} ; p\right)\left(R_{n+1}-R_{n}\right) \\
& +\frac{\theta\left(q^{-n}, \varepsilon_{6} q^{n} / \varepsilon_{8}, \varepsilon_{6} q^{1+n} / \varepsilon_{8} ; p\right)}{\theta\left(q^{1-2 n} \varepsilon_{8} / A, q^{2-2 n} \varepsilon_{8} / A ; p\right)} \\
& \quad \times \prod_{r=1}^{3} \theta\left(\frac{\varepsilon_{r} \varepsilon_{6} \varepsilon_{8} q^{1-n}}{A} ; p\right) \theta\left(\frac{q^{2-n} x^{ \pm 1}}{A} ; p\right)\left(R_{n-1}-R_{n}\right) \\
& +\theta\left(\frac{\varepsilon_{6} q^{n}}{A}, \varepsilon_{6} x^{ \pm 1} ; p\right) \prod_{r=1}^{3} \theta\left(\frac{q}{\varepsilon_{r} \varepsilon_{8}} ; p\right) R_{n}=0
\end{aligned}
$$

с начальными условиями $R_{-1}=0$ и $R_{0}=1$.

Введем функции

$$
z(x)=\frac{\theta\left(x \xi^{ \pm 1} ; p\right)}{\theta\left(x \eta^{ \pm 1} ; p\right)}, \quad \alpha_{n}=z\left(\frac{q^{n}}{\varepsilon_{8}}\right), \quad \beta_{n}=z\left(A q^{n-1}\right),
$$

где $\xi$ и $\eta$ - произвольные калибровочные параметры, $\xi \neq \eta^{ \pm 1} p^{k}, k \in \mathbb{Z}$. Тогда соотношение (7.5) может быть переписано в более структурированном виде

$$
\begin{aligned}
& \left(z(x)-\alpha_{n+1}\right) \rho\left(\frac{A q^{n-1}}{\varepsilon_{8}}\right)\left(R_{n+1}(x ; q, p)-R_{n}(x ; q, p)\right) \\
& +\left(z(x)-\beta_{n-1}\right) \rho\left(q^{-n}\right)\left(R_{n-1}(x ; q, p)-R_{n}(x ; q, p)\right) \\
& \quad+\delta\left(z(x)-z\left(\varepsilon_{6}\right)\right) R_{n}(x ; q, p)=0,
\end{aligned}
$$

где

$$
\begin{aligned}
\rho(t) & =\frac{\theta\left(t, \varepsilon_{6} /\left(\varepsilon_{8} t\right), q \varepsilon_{6} /\left(\varepsilon_{8} t\right), q t /\left(\varepsilon_{1} \varepsilon_{2}\right), q t /\left(\varepsilon_{2} \varepsilon_{3}\right), q t /\left(\varepsilon_{1} \varepsilon_{3}\right), q^{2} t \eta^{ \pm 1} / A ; p\right)}{\theta\left(q t^{2} \varepsilon_{8} / A, q^{2} t^{2} \varepsilon_{8} / A ; p\right)}, \\
\delta & =\theta\left(\frac{q^{2} \varepsilon_{6}}{A}, \frac{q}{\varepsilon_{1} \varepsilon_{8}}, \frac{q}{\varepsilon_{2} \varepsilon_{8}}, \frac{q}{\varepsilon_{3} \varepsilon_{8}}, \varepsilon_{6} \eta^{ \pm 1} ; p\right) .
\end{aligned}
$$

Начальные условия $R_{-1}=0$ и $R_{0}=1$ гарантируют, что $R_{n}(x ; q, p)$ являются рациональными функциями $z(x)$ с полюсами в точках $\alpha_{1}, \ldots, \alpha_{n}$ (т. е. вся зависимость от $x$ входит в $R_{n}$ только через переменную $\left.z(x)\right)$. 
Предположим, что $\phi_{\lambda}$ является решением абстрактной обобщенной спектральной задачи $\mathscr{D}_{1} \phi_{\lambda}=\lambda \mathscr{D}_{2} \phi_{\lambda}$ с некоторыми операторами $\mathscr{D}_{1,2}$. Пусть задано также некоторое скалярное произведению $\langle\psi \mid \phi\rangle$, которое определяет формальные сопряженные операторы $\mathscr{D}_{1,2}^{T}$ по обычному правилу $\left\langle\mathscr{D}_{1,2}^{T} \psi \mid \phi\right\rangle=\langle\psi|$ $\left.\mathscr{D}_{1,2} \phi\right\rangle$. Пусть $\psi_{\lambda}$ - решения дуальной обобщенной задачи на собственные значения $\mathscr{D}_{1}^{T} \psi_{\lambda}=\lambda \mathscr{D}_{2}^{T} \psi_{\lambda}$. Тогда $0=\left\langle\psi_{\mu} \mid\left(\mathscr{D}_{1}-\lambda \mathscr{D}_{2}\right) \phi_{\lambda}\right\rangle=(\mu-\lambda)\left\langle\mathscr{D}_{2}^{T} \psi_{\mu} \mid \phi_{\lambda}\right\rangle$, т. е. функция $\mathscr{D}_{2}^{T} \psi_{\mu}$ ортогональна $\phi_{\lambda}$ при $\mu \neq \lambda$. Следствия этого хорошо известного в линейной алгебре факта детально исследованы Жедановым [70] в случае, когда $\mathscr{D}_{1,2}$ являются матрицами Якоби (т. е. трехдиагональными матрицами). В частности, было показано, что такие обобщенные задачи на собственные значения эквивалентны теории биортогональных рациональных функций, обобщающих ортогональные многочлены. Они связаны также с рекуррентным соотношением типа $R_{\mathrm{II}}$, изучавшимся в [71], и с соотношениями ортогональности, появляющимися в теории многоточечной аппроксимации Паде [72], [73]. Рекуррентное соотношение (7.6) попадает в этот класс задач, так что существует линейный функционал $\mathscr{L}$ с условием $\mathscr{L}\left\{T_{m}(x ; q, p) R_{n}(x ; q, p)\right\}=h_{n} \delta_{n m}$ для некоторых рациональных функций $T_{m}$ и нормировочных постоянных $h_{n}$.

Эллиптическое гипергеометрическое уравнение для $R_{n}$-функций может быть записано в виде обобщенной задачи на собственные значения [11]:

$$
\mathscr{D}\left(\varepsilon_{4}^{\prime}, \varepsilon_{5}^{\prime}\right) R_{n}=\lambda_{n} \mathscr{D}\left(\varepsilon_{4}^{\prime \prime}, \varepsilon_{5}^{\prime \prime}\right) R_{n}
$$

где

$$
\mathscr{D}\left(\varepsilon_{4}, \varepsilon_{5}\right)=A(x)\left(T_{q, x}-1\right)+A\left(x^{-1}\right)\left(T_{q, x}^{-1}-1\right)+\nu, \quad T_{q, x} f(x)=f(q x),
$$

обозначает оператор, с помощью которого можно переписать равенство (5.10) в виде $\mathscr{D}\left(\varepsilon_{4}, \varepsilon_{5}\right) f(x)=0$. Штрихованные параметры произвольны при ограничении $\varepsilon_{4}^{\prime} \varepsilon_{5}^{\prime}=\varepsilon_{4}^{\prime \prime} \varepsilon_{5}^{\prime \prime}=\varepsilon_{4} \varepsilon_{5}$, а остальные параметры остаются неизменными (поэтому зависимость от них не указывается). Спектральная переменная

$$
\lambda_{n}=\frac{\theta\left(\varepsilon_{4} / \varepsilon_{4}^{\prime}, \varepsilon_{4} / \varepsilon_{5}^{\prime} ; p\right)}{\theta\left(\varepsilon_{4} / \varepsilon_{4}^{\prime \prime}, \varepsilon_{4} / \varepsilon_{5}^{\prime \prime} ; p\right)}
$$

дискретна, так как $\varepsilon_{4}=p q^{n+1} / \varepsilon_{8}$. Если взять в качестве функционала $\mathscr{L}$ свертку, ядро которой совпадает с ядром эллиптического бета-интеграла,

$$
\mathscr{L}\{\phi(x) \psi(x)\}=\kappa \int_{\mathbb{T}} \frac{\prod_{j=1,2,3,6,8} \Gamma\left(\varepsilon_{j} x^{ \pm 1} ; p, q\right)}{\Gamma\left(x^{ \pm 2}, A x^{ \pm 1} ; p, q\right)} \phi(x) \psi(x) \frac{d x}{x},
$$

то функции

$$
T_{n}(x ; q, p)={ }_{12} V_{11}\left(\frac{A \varepsilon_{6}}{q} ; \frac{A}{\varepsilon_{1}}, \frac{A}{\varepsilon_{2}}, \frac{A}{\varepsilon_{3}}, \varepsilon_{6} x, \frac{\varepsilon_{6}}{x}, q^{-n}, \frac{A q^{n-1}}{\varepsilon_{8}} ; q, p\right)
$$

служат аналогом $\mathscr{D}_{2}^{T} \psi_{\mu}$ для $R_{n}(x ; q, p)$. Функции $T_{n}(x ; q, p)$ являются рациональными функциями $z(x)$ с полюсами в точках $\beta_{1}, \ldots, \beta_{n}$ и получаются из $R_{n}(x ; q, p)$ после замены параметра $\varepsilon_{8} \rightarrow p q / A$ (при этом зависимость от $p$ в параметрах исчезает из-за эллиптичности ${ }_{12} V_{11}$-ряда по ним). 
Обозначим $R_{n m}(x):=R_{n}(x ; q, p) R_{m}(x ; p, q)$ и $T_{n m}(x):=T_{n}(x ; q, p) T_{m}(x ; p, q)$, где все ${ }_{12} V_{11}$-ряды одновременно обрываются благодаря модифицированному условию обрыва $\varepsilon_{4} \varepsilon_{8}=p^{m+1} q^{n+1}, n, m=0,1, \ldots$ Так как $R_{m}(q x ; p, q)=$ $R_{m}(x ; p, q)$, функции $R_{n m}$ являются решениями уже не одной, а двух обобщенных задач на собственные значения, отличающихся перестановкой $p$ и $q$. Поэтому соотношения ортогональности в нашем случае оказываются более сложными, чем для биортогональных рациональных функций.

Теорема 6. Функции $R_{n m}(x)$ и $T_{n m}(x)$ удовлетворяют следующему соотношению двухиндексной биортогональности:

$$
\kappa \int_{C_{m n, k l}} T_{n l}(x) R_{m k}(x) \frac{\prod_{j \in S} \Gamma\left(\varepsilon_{j} x^{ \pm 1} ; p, q\right)}{\Gamma\left(x^{ \pm 2}, A x^{ \pm 1} ; p, q\right)} \frac{d x}{x}=h_{n l} \delta_{m n} \delta_{k l},
$$

где $S=\{1,2,3,6,8\}, C_{m n, k l}$ обозначает контур, отделяющий последовательности точек $\varepsilon_{j} p^{a} q^{b}(j=1,2,3,6), \varepsilon_{8} p^{a-k} q^{b-m}, p^{a+1-l} q^{b+1-n} / A, a, b=0,1$, $2, \ldots$, от их $x \rightarrow x^{-1}$ партнеров, а нормировочные постоянные имент вид

$$
\begin{aligned}
h_{n l} & =\frac{\prod_{j<k, j, k \in S} \Gamma\left(\varepsilon_{j} \varepsilon_{k} ; p, q\right)}{\prod_{j \in S} \Gamma\left(A \varepsilon_{j}^{-1} ; p, q\right)} h_{n}(q, p) \cdot h_{l}(p, q), \\
h_{n}(q, p) & =\frac{\theta\left(A /\left(q \varepsilon_{8}\right) ; p\right) \theta\left(q, q \varepsilon_{6} / \varepsilon_{8}, \varepsilon_{1} \varepsilon_{2}, \varepsilon_{1} \varepsilon_{3}, \varepsilon_{2} \varepsilon_{3}, A \varepsilon_{6}\right)_{n} q^{-n}}{\theta\left(A q^{2 n} /\left(q \varepsilon_{8}\right) ; p\right) \theta\left(1 /\left(\varepsilon_{6} \varepsilon_{8}\right), \varepsilon_{1} \varepsilon_{6}, \varepsilon_{2} \varepsilon_{6}, \varepsilon_{3} \varepsilon_{6}, A /\left(q \varepsilon_{6}\right), A /\left(q \varepsilon_{8}\right)\right)_{n}} .
\end{aligned}
$$

Прямое доказательство этого утверждения путем непосредственного вычисления интеграла в левой части с помощью формулы (3.1) и суммы ФренкеляТураева дано в работе [11]. Возникновение таких двухиндексных соотношений ортогональности для функций одной переменной - новое явление в теории специальных функций. Отметим, что $R_{n m}(x)$ и $T_{n m}(x)$ являются мероморфными функциями $x \in \mathbb{C}^{*}$ с существенными особенностями при $x=0, \infty$; только при $k=l=0$ или $n=m=0$ они становятся рациональными функциями некоторого аргумента, зависящего от $x$. При $k=l=0$ можно перейти к пределу $p \rightarrow 0$ при фиксированных параметрах и получить функции $R_{n}(x ; q, 0)$ и $T_{n}(x ; q, 0)$, которые совпадают с семейством непрерывных $10 \varphi_{9}$-биортогональных рациональных функций Рахмана [39]. Дальнейшее вырождение этих функций приводит к многочленам Аски-Вильсона [41]. Дополнительное ограничение на один из параметров в $R_{n}(x ; q, p)$ и $T_{n}(x ; q, p)$ приводит к конечномерной системе биортогональных рациональных функций дискретного аргумента [9], [74], которые обобщают функции Вильсона [75]. Элементарный подход к анализу этих функций, связанных с эллиптическими 6j-символами [8], предложен Розенгреном в [76]. Некоторые свойства функций $R_{n}(x ; q, p)$ изучены в недавней работе [48].

Можно построить соотношение, аналогичное (7.8), на основе модифицированного эллиптического бета-интеграла (3.10) [31]. Для этого необходимо воспользоваться параметризацией базисных переменных через квазипериоды $\omega_{1,2,3}$ и перейти к функциям $r_{n}\left(u ; \omega_{1}, \omega_{2}, \omega_{3}\right)=R_{n}\left(e^{2 \pi i u / \omega_{2}} ; e^{2 \pi i \omega_{1} / \omega_{2}}, e^{2 \pi i \omega_{3} / \omega_{2}}\right)$, где также сделана подстановка $\varepsilon_{j}=e^{2 \pi i g_{j} / \omega_{2}}$. Аналогичным образом необходимо переобозначить $T_{n}(x ; q, p)$ через $s_{n}\left(u ; \omega_{1}, \omega_{2}, \omega_{3}\right)$, а $h_{n}(q, p)$ через $h_{n}\left(\omega_{1}, \omega_{2}, \omega_{3}\right)$. Произведения

$$
r_{n m}(u)=r_{n}\left(u ; \omega_{1}, \omega_{2}, \omega_{3}\right) r_{m}\left(u ; \omega_{2}, \omega_{1}, \omega_{3}\right)
$$




$$
s_{n m}(u)=s_{n}\left(u ; \omega_{1}, \omega_{2}, \omega_{3}\right) s_{m}\left(u ; \omega_{2}, \omega_{1}, \omega_{3}\right)
$$

инвариантны относительно перестановок $\omega_{1} \leftrightarrow \omega_{2}, n \leftrightarrow m$. Тогда для специально подобранного контура $\widetilde{C}_{m n, k l}$ имеем

$$
\tilde{\kappa} \int_{\widetilde{C}_{m n, k l}} s_{n l}(u) r_{m k}(u) \frac{\prod_{j \in S} G\left(g_{j} \pm u ; \boldsymbol{\omega}\right)}{G( \pm 2 u, \mathscr{A} \pm u ; \boldsymbol{\omega})} \frac{d u}{\omega_{2}}=\tilde{h}_{n l} \delta_{m n} \delta_{k l},
$$

где $\mathscr{A}=\sum_{j \in S} g_{j}$ и

$$
\tilde{h}_{n l}=\frac{\prod_{j<m, j, m \in S} G\left(g_{j}+g_{m} ; \boldsymbol{\omega}\right)}{\prod_{j \in S} G\left(\mathscr{A}-g_{j} ; \boldsymbol{\omega}\right)} h_{n}\left(\omega_{1}, \omega_{2}, \omega_{3}\right) h_{l}\left(\omega_{2}, \omega_{1}, \omega_{3}\right) .
$$

В отличие от предыдущего случая, предельный переход на $q$-гипергеометрический уровень $\operatorname{Im}\left(\omega_{3} / \omega_{2}\right), \operatorname{Im}\left(\omega_{3} / \omega_{1}\right) \rightarrow+\infty$ (т. е. $\left.p, r \rightarrow 0\right)$ корректно определен и сохраняет двухиндексную структуру соотношений биортогональности. В частности, при этом $r_{n m}(u)$-функция вырождается в произведение двух $q$-гипергеометрических рядов

$$
\begin{gathered}
r_{n m}\left(u ; \omega_{1}, \omega_{2}\right)={ }_{10} W_{9}\left(e^{2 \pi i\left(g_{6}-g_{8}\right) / \omega_{2}} ; e^{2 \pi i\left(\omega_{1}-g_{1}-g_{8}\right) / \omega_{2}}, \ldots, e^{2 \pi i\left(g_{6}+u\right) / \omega_{2}} ; q, q\right) \\
\times{ }_{10} W_{9}\left(e^{2 \pi i\left(g_{6}-g_{8}\right) / \omega_{1}} ; e^{2 \pi i\left(\omega_{2}-g_{1}-g_{8}\right) / \omega_{1}}, \ldots, e^{2 \pi i\left(g_{6}+u\right) / \omega_{1}} ; \tilde{q}^{-1}, \tilde{q}^{-1}\right),
\end{gathered}
$$

базисные переменные которых связаны модулярным преобразованием, а нормировка меры задается интегралом (3.13).

7.2. Обрывающаяся цепная дробь. В работе [46] вычислена обрывающаяся цепная дробь, связанная с рациональными функциями $R_{n}(x ; q, p)$. Пусть $U_{n}$ и $V_{n}$ - числовые последовательности, удовлетворяющие трехчленному рекуррентному соотношению

$$
\psi_{n+1}=\xi_{n} \psi_{n}+\eta_{n} \psi_{n-1}, \quad n=1,2, \ldots,
$$

с некоторыми коэффициентами $\xi_{n}$ и $\eta_{n}$ и начальным условиям $U_{0}=0, U_{1}=1$ и $V_{0}=1, V_{1}=\xi_{0}$. Известно, что их отношение связано с конечной цепной дробью

$$
\frac{U_{n}}{V_{n}}=\frac{1}{\xi_{0}+\frac{\eta_{1}}{\xi_{1}+\ddots_{+} \frac{\eta_{n-1}}{\xi_{n-1}}}}, \quad n=1,2, \ldots
$$

Определим многочлены $z(x) n$-й степени:

$$
P_{n}(z(x))=\kappa_{n} \prod_{k=1}^{n}\left(z-\alpha_{k}\right) R_{n}(x ; q, p), \quad \kappa_{n}=\prod_{j=0}^{n-1} \rho\left(a q^{j-1}\right),
$$

и положим $P_{0}(z(x))=1$. Заменяя $R_{n}(x ; q, p)$ на $P_{n}(z(x))$ в $(7.6)$, получаем следующее трехчленное рекуррентное соотношение:

$$
P_{n+1}(z)+\left(v_{n}-\rho_{n} z\right) P_{n}(z)+u_{n}\left(z-\alpha_{n}\right)\left(z-\beta_{n-1}\right) P_{n-1}(z)=0
$$


с начальными условиями $P_{-1}=0, P_{0}=1$ и рекуррентными коэффициентами

$$
\begin{gathered}
u_{n}=\rho\left(\frac{1}{q^{n}}\right) \rho\left(\frac{A q^{n-2}}{\varepsilon_{8}}\right), \quad \rho_{n}=\rho\left(\frac{A q^{n-1}}{\varepsilon_{8}}\right)+\rho\left(\frac{1}{q^{n}}\right)-\delta, \\
v_{n}=\alpha_{n+1} \rho\left(\frac{A q^{n-1}}{\varepsilon_{8}}\right)+\beta_{n-1} \rho\left(\frac{1}{q^{n}}\right)-\delta z\left(\varepsilon_{6}\right) .
\end{gathered}
$$

В этом случае $V_{n}=P_{n}(z)$, а $U_{n}=P_{n-1}^{(1)}(z)$ - ассоциированные многочлены, имеющие степень $n-1$ по $z$.

Предположим, что многочлен $P_{N+1}(z)$ имеет только простые нули, т. е.

$$
P_{N+1}\left(z_{s}\right)=0, \quad z_{s} \equiv z_{s}^{(N+1)}, \quad s=0,1, \ldots, N, \quad z_{s} \neq z_{s^{\prime}} \text { при } s \neq s^{\prime} .
$$

Тогда соответствующая цепная дробь может быть разложена в простую дробь (как рациональная функция $z$ )

$$
\frac{P_{N}^{(1)}(z)}{P_{N+1}(z)}=\sum_{s=0}^{N} \frac{g_{s}}{z-z_{s}}, \quad g_{s}=\frac{P_{N}^{(1)}\left(z_{s}\right)}{P_{N+1}^{\prime}\left(z_{s}\right)} .
$$

Казоратиан любых двух решений $U_{n}$ и $V_{n}$ для (7.10) удовлетворяет соотношению

$$
U_{n+1} V_{n}-U_{n} V_{n+1}=(-1)^{n} \eta_{1} \cdots \eta_{n}\left(U_{1} V_{0}-U_{0} V_{1}\right),
$$

которое дает

$$
P_{n}(z) P_{n}^{(1)}(z)-P_{n+1}(z) P_{n-1}^{(1)}(z)=h_{n} A_{n}(z) B_{n}(z)
$$

где $h_{n}=u_{1} u_{2} \cdots u_{n}$ и

$$
A_{n}(z)=\prod_{i=1}^{n}\left(z-\alpha_{i}\right), \quad B_{n}(z)=\prod_{i=1}^{n}\left(z-\beta_{i-1}\right) .
$$

Зафиксировав $n=N$ и положив $z=z_{s}, s=0,1, \ldots, N$, в (7.15), можно выразить $P_{N}^{(1)}\left(z_{s}\right)$ через $P_{N}\left(z_{s}\right), h_{N}, A_{N}\left(z_{s}\right)$ и $B_{N}\left(z_{s}\right)$. Это приводит к следующему удобному для вычислений выражению для вычетов полюсов $g_{s}$ в (7.14):

$$
g_{s}=\frac{h_{N} A_{N}\left(z_{s}\right) B_{N}\left(z_{s}\right)}{P_{N+1}^{\prime}\left(z_{s}\right) P_{N}\left(z_{s}\right)} .
$$

В пределе

$$
\varepsilon_{3} \varepsilon_{6}=q^{-N+\epsilon}, \quad N=0,1,2, \ldots, \quad \epsilon \rightarrow 0
$$

получаем, что $u_{N+1} \rightarrow 0$, и цепная дробь обрывается автоматически. Оказывается, что эта дробь вычисляется в замкнутом виде по формуле (7.14). При $\epsilon \rightarrow 0$ рациональная функция $R_{N+1}(x ; q, p)$ расходится, так как эллиптический символ Похгаммера $\theta\left(\varepsilon_{3} \varepsilon_{6}\right)_{N+1}$ в знаменателе последнего коэффициента ${ }_{12} V_{11}$-ряда стремится к нулю. Но одновременно имеем $\kappa_{N+1} \rightarrow 0$, так что многочлен $P_{N+1}(z)$ принимает конечное значение, и его нули находятся в явном виде: $z_{s}=z\left(\varepsilon_{6} q^{s}\right), s=0,1, \ldots, N$. При этом оказывается, что многочлен $P_{N}\left(z_{s}\right)$ вычисляется в замкнутом виде по формуле суммирования Френкеля-Тураева. 
Остальные величины, определяющие вычеты $g_{s}$, находятся достаточно легко, хотя и имеют громоздкие выражения.

Предположим, что выполнены условия простоты нулей $z_{s}$ и другие ограничения на параметры, гарантирующие $u_{k} \neq 0$ при $k=1, \ldots, N$, описание которых мы пропускаем. Тогда обрывающаяся эллиптическая гипергеометрическая цепная дробь имеет следующее явное представление:

$$
\begin{aligned}
& \frac{1}{\rho_{0} z-v_{0}-\frac{u_{1}\left(z-\alpha_{1}\right)\left(z-\beta_{0}\right)}{\rho_{1} z-v_{1}-\ddots_{-} \frac{u_{N}\left(z-\alpha_{N}\right)\left(z-\beta_{N-1}\right)}{\rho_{N} z-v_{N}}}} \\
& =\frac{1}{\left(z\left(\varepsilon_{6}\right)-z(x)\right) \delta}{ }_{12} V_{11}\left(\frac{q \varepsilon_{6}}{\varepsilon_{8}} ; q, \varepsilon_{6} \varepsilon_{1}, \varepsilon_{6} \varepsilon_{2}, \frac{q x}{\varepsilon_{8}}, \frac{q}{\varepsilon_{8} x}, q^{-N}, \frac{\varepsilon_{6} q^{N+2}}{\varepsilon_{1} \varepsilon_{2} \varepsilon_{8}} ; q, p\right) \text {, }
\end{aligned}
$$

где в выражения для всех рекуррентных коэффициентов, включая $\delta$, необходимо подставить $\varepsilon_{3}=q^{-N} / \varepsilon_{6}$ и $z(x)=\theta\left(x \xi^{ \pm 1} ; p\right) / \theta\left(x \eta^{ \pm 1} ; p\right)$. В [46] этот результат был представлен в другой (аддитивной) системе обозначений.

Эта формула описывает наиболее общую обрывающуюся цепную дробь гипергеометрического типа, найденную к настоящему времени. При фиксированных параметрах в пределе $p \rightarrow 0$ получается обрывающаяся цепная дробь Гупты-Массона [77] (см. следствие 3.3), описываемая совершенно уравновешенным сбалансированным 10 $\varphi_{9}$-рядом. Дальнейшая спецификация параметров приводит к цепной дроби Ватсона, которая в свою очередь является $q$-аналогом известной цепной дроби Рамануджана (см. детали в [78]).

7.3. Непрерывная биортогональность $V$-функции. Описанные выше соотношения (7.8) соответствуют дискретным значениям одного из параметров в эллиптическом гипергеометрическом уравнении. В работе [79] показано, что $V$-функция с общим набором непрерывных параметров обладает также соотношениями биортогональности, характерными для непрерывных спектров.

Рассмотрим случай $m=2$ в рекурсии (6.11). После задания ограничений на параметры $t_{5} t_{7}=t_{6} t_{8}=p q$ интеграл в левой части явно вычисляется. Тогда после ряда переобозначений и использования преобразования (5.2) возникает равенство

$$
\phi(x ; c, d \mid \xi ; s)=\kappa \int_{\mathbb{T}} R(c, d, a, b ; x, w \mid s) \phi(w ; a, b \mid \xi ; s) \frac{d w}{w},
$$

где базисные векторы имеют вид

$$
\phi(w ; a, b \mid \xi ; s)=\Gamma\left(s a \xi^{ \pm 1}, s b \xi^{ \pm 1}, \sqrt{\frac{p q}{a b}} w^{ \pm 1} \xi^{ \pm 1} ; p, q\right)
$$

и

$$
\begin{aligned}
& R(c, d, a, b ; x, w \mid s)=\left(\Gamma\left(\frac{p q}{a b}, \frac{a b}{p q}, w^{ \pm 2} ; p, q\right)\right)^{-1} \\
& \quad \times V\left(s c, s d, \sqrt{\frac{p q}{c d}} x, \varepsilon \sqrt{\frac{p q}{c d}} x^{-1}, \frac{p q}{a s}, \frac{p q}{b s}, \sqrt{\frac{a b}{p q}} w, \sqrt{\frac{a b}{p q}} w^{-1}\right) .
\end{aligned}
$$


Здесь параметры $a, b, c, d, s$ и $x, \xi$ произвольны, но их выбор должен быть согласован с условием, что фигурирующие контуры интегрирования разделяют сходящиеся к нулю и уходящие на бесконечность последовательности полюсов подынтегральных выражений. Переменная $\xi$ входит только в базисные векторы $\phi(w ; a, b \mid \xi ; s)$, и ядро $R$ может рассматриваться как "матрица вращения" с непрерывными индексами $x$ и $w$, позволяющая менять произвольным образом параметры $a$ и $b$. Равенство (7.21) является интегральным обобщением соотношения, использованного в [76] Розенгреном для вывода свойств эллиптических $6 j$-символов.

Обозначая $w=e^{i \theta}, y=\cos \theta$ и пользуясь равенством

$$
\int_{\mathbb{T}} f(\cos \theta) \frac{d w}{i w}=\int_{0}^{2 \pi} f(\cos \theta) d \theta=2 \int_{-1}^{1} f(y) \frac{d y}{\sqrt{1-y^{2}}},
$$

можно записать

$$
\phi(x ; c, d \mid \xi ; s)=2 i \kappa \int_{-1}^{1} R\left(c, d, a, b ; x, e^{i \theta} \mid s\right) \phi\left(e^{i \theta} ; a, b \mid \xi ; s\right) \frac{d y}{\sqrt{1-y^{2}}} .
$$

В пределе $c \rightarrow a$ и $d \rightarrow b$ возникает следующее соотношение в смысле обобщенных функций:

$$
\lim _{c \rightarrow a, d \rightarrow b} R\left(c, d, a, b ; e^{i \varphi}, e^{i \theta}\right)=\frac{2 \pi \sqrt{1-y^{2}}}{(p ; p)_{\infty}(q ; q)_{\infty}} \delta(v-y),
$$

где $v=\cos \varphi$.

Двукратное использование (7.19) с различными параметрами очевидным образом приводит к свойству самовоспроизведения для ядра

$$
\kappa \int_{\mathbb{T}} R(a, b, c, d ; x, w \mid s) R(c, d, e, f ; w, z \mid s) \frac{d w}{w}=R(a, b, e, f ; x, z \mid s)
$$

и соотношению биортогональности

$$
\int_{-1}^{1} R\left(a, b, c, d ; e^{i \varphi}, e^{i \theta} \mid s\right) R\left(c, d, a, b ; e^{i \theta}, e^{i \varphi^{\prime}} \mid s\right) \frac{d y}{\sqrt{1-y^{2}}}=\frac{\sqrt{1-v^{2}}}{(2 i \kappa)^{2}} \delta\left(v-v^{\prime}\right)
$$

где $v=\cos \varphi$ и $v^{\prime}=\cos \varphi^{\prime}$. Подстановка выражения для $R$-функций $(7.21)$ в (7.25) приводит к равенству

$$
\begin{gathered}
\int_{-1}^{1} \frac{1}{\Gamma\left(e^{ \pm 2 i \theta} ; p, q\right)} V\left(s a, s b, \sqrt{\frac{p q}{a b}} e^{i \varphi}, \sqrt{\frac{p q}{a b}} e^{-i \varphi}, \frac{p q}{c}, \frac{p q}{d}, \sqrt{\frac{c d}{\rho}} e^{i \theta}, \sqrt{\frac{c d}{\rho}} e^{-i \theta}\right) \\
\quad \times V\left(s c, s d, \sqrt{\frac{p q}{c d}} e^{i \theta}, \sqrt{\frac{p q}{c d}} e^{-i \theta}, \frac{p q}{a s}, \frac{p q}{b s}, \sqrt{\frac{a b}{p q}} e^{i \varphi^{\prime}}, \sqrt{\frac{a b}{p q}} e^{-i \varphi^{\prime}}\right) \frac{d y}{\sqrt{1-y^{2}}} \\
=\Gamma\left(\frac{a b}{p q}, \frac{p q}{a b}, \frac{c d}{p q}, \frac{p q}{c d}, e^{ \pm 2 i \varphi} ; p, q\right) \frac{\sqrt{1-v^{2}}}{(2 i \kappa)^{2}} \delta\left(v-v^{\prime}\right) .
\end{gathered}
$$

Параметры $v$ и $v^{\prime}$ могут рассматриваться как непрерывные спектральные переменные в операторной формулировке эллиптического гипергеометрического уравнения. Поэтому соотношение (7.26) должно вытекать из этого уравнения, но точная связь между ними еще не установлена. 


\section{8. Связь с алгеброй Склянина}

В [34] Рэйнс ввел интересный конечно-разностный оператор, связанный с системой корней $B C_{n}$. При $n=1$ он может быть представлен в виде

$$
D(a, b, c, d ; p ; q)=\frac{\theta(a z, b z, c z, d z ; p)}{z \theta\left(z^{2} ; p\right)} T_{z, q}^{1 / 2}+\frac{\theta\left(a z^{-1}, b z^{-1}, c z^{-1}, d z^{-1} ; p\right)}{z^{-1} \theta\left(z^{-2} ; p\right)} T_{z, q}^{-1 / 2},
$$

где $T_{z, q}^{ \pm 1 / 2} f(z)=f\left(q^{ \pm 1 / 2} z\right)$ - оператор $q$-сдвига и $a, b, c, d$ - произвольные параметры. Позднее Рэйнс также заметил [35], [80], что этот оператор эквивалентен общей линейной комбинации четырех образующих алгебры Склянина $S_{0}, \ldots, S_{3}$ [81], [82].

Определяющие соотношения алгебры Склянина имеют вид

$$
\begin{aligned}
S_{\alpha} S_{\beta}-S_{\beta} S_{\alpha} & =i\left(S_{0} S_{\gamma}+S_{\gamma} S_{0}\right), \\
S_{0} S_{\alpha}-S_{\alpha} S_{0} & =i J_{\beta \gamma}\left(S_{\beta} S_{\gamma}+S_{\gamma} S_{\beta}\right),
\end{aligned}
$$

где $J_{\beta \gamma}$ - структурные постоянные алгебры, а $(\alpha, \beta, \gamma)$ - произвольная циклическая перестановка $(1,2,3)$. В [82] найдено представление $S_{a}$ в виде разностных операторов:

$$
S_{a}=i^{\delta_{a, 2}} \frac{\theta_{a+1}(\eta \mid \tau)}{\theta_{1}(2 u \mid \tau)}\left(\theta_{a+1}(2 u-2 g \mid \tau) e^{\eta \partial_{u}}-\theta_{a+1}(-2 u-2 g \mid \tau) e^{-\eta \partial_{u}}\right),
$$

где $e^{ \pm \eta \partial_{u}} f(u)=f(u \pm \eta)$, и при условии квантования $g=\ell \eta, \ell=0,1 / 2,1, \ldots$, описано их действие на пространстве четных тэта-функций порядка $4 \ell$. Комбинация образующих

$$
\begin{aligned}
2 \Delta\left(a_{1}, a_{2}, a_{3}, a_{4}\right):= & \frac{\prod_{j=1}^{3} \theta_{1}\left(a_{j}+a_{4}+2 g\right)}{\theta_{1}(\eta)} S_{0}-\frac{\prod_{j=1}^{3} \theta_{1}\left(a_{j}+a_{4}+2 g+\frac{1}{2}\right)}{\theta_{1}\left(\eta+\frac{1}{2}\right)} S_{1} \\
& -i e^{\pi i\left(\frac{\tau}{2}+2 a_{4}+2 g-\eta\right)} \frac{\prod_{j=1}^{3} \theta_{1}\left(a_{j}+a_{4}+2 g+\frac{1+\tau}{2}\right)}{\theta_{1}\left(\eta+\frac{1+\tau}{2}\right)} S_{2} \\
& +e^{\pi i\left(\frac{\tau}{2}+2 a_{4}+2 g-\eta\right)} \frac{\prod_{j=1}^{3} \theta_{1}\left(a_{j}+a_{4}+2 g+\frac{\tau}{2}\right)}{\theta_{1}\left(\eta+\frac{\tau}{2}\right)} S_{3}
\end{aligned}
$$

с нормировкой $\sum_{j=1}^{4} a_{j}=-4 g$ представима в виде [80]

$$
\Delta\left(a_{1}, a_{2}, a_{3}, a_{4}\right)=\frac{\prod_{j=1}^{4} \theta_{1}\left(a_{j}+u\right)}{\theta_{1}(2 u)} e^{\eta \partial_{u}}+\frac{\prod_{j=1}^{4} \theta_{1}\left(a_{j}-u\right)}{\theta_{1}(-2 u)} e^{-\eta \partial_{u}} .
$$

При переходе к мультипликативной системе обозначений

$$
(a, b, c, d):=e^{2 \pi i a_{1,2,3,4}}, \quad \rho:=a b c d=e^{-8 \pi i g}, \quad z:=e^{2 \pi i u}, \quad q:=e^{4 \pi i \eta}
$$

возникает указанный выше оператор (8.1):

$$
\Delta\left(a_{1}, a_{2}, a_{3}, a_{4}\right)=\left(i p^{1 / 8}(p ; p)_{\infty}\right)^{3} e^{4 \pi i g} D(a, b, c, d ; p ; q) .
$$


Стандартная задача на собственные значения $D \psi=\lambda \psi$ оказывается очень сложной, так как представляет собой разностный аналог уравнения Гойна [79]. С одной стороны, известно, что задача на собственные значения для одночастичного гамильтониана модели Иноземцева [65] эквивалентна уравнению Гойна. С другой стороны, классические уравнения движения для этого гамильтониана с модулярным параметром $\tau$, рассматриваемым как переменная времени, приводят к уравнению Пенлеве VI [83]. Сам $D$-оператор оказывается связанным с моделью ван Диежена [62], [79]. Все это и связь с эллиптическими гипергеометрическими функциями, описанная ниже, демонстрируют некоторую математическую универсальность оператора (8.1).

Рассмотрим обобщенную задачу на собственные значения вида

$$
\begin{aligned}
& D(a, b, c, d ; p ; q) f\left(z ; w ; q^{1 / 2} a, q^{1 / 2} b ; \rho\right) \\
& \quad=\lambda(w) D\left(a, b, c^{\prime}, d^{\prime} ; p ; q\right) f\left(z ; w ; q^{1 / 2} a, q^{1 / 2} b ; \rho\right),
\end{aligned}
$$

где $c d=c^{\prime} d^{\prime}$. Это уравнение решается в явном виде. Используя параметризацию

$$
\lambda(w)=\frac{\theta(w \sqrt{c / d}, w \sqrt{d / c} ; p)}{\theta\left(w \sqrt{c^{\prime} / d^{\prime}}, w \sqrt{d^{\prime} / c^{\prime}} ; p\right)},
$$

при $|q|<1$ получаем [79]

$$
f(z ; w ; a, b ; \rho)=\Gamma\left(\frac{p q}{a} z^{ \pm 1}, \frac{p q}{b} z^{ \pm 1}, \sqrt{\frac{a b}{\rho}} w^{ \pm 1} z^{ \pm 1} ; p, q\right),
$$

с точностью до умножения на произвольную функцию $\varphi(z), \varphi(q z)=\varphi(z)$. Очевидно, что функция (8.4) совпадает с базисным вектором (7.20) после переобозначения параметров.

Для инвариантных относительно преобразования $z \rightarrow z^{-1}$ функций, $\psi(z)=$ $\psi\left(z^{-1}\right)$, определим скалярное произведение

$$
\langle\chi(z), \psi(z)\rangle=\kappa \int_{\mathbb{T}} \frac{\chi(z) \psi(z)}{\Gamma\left(z^{ \pm 2} ; p, q\right)} \frac{d z}{z} .
$$

Тогда формально сопряженный к $D$ оператор имеет вид

$$
D^{*}(a, b, c, d ; p ; q)=\frac{c d}{q^{1 / 2}} D\left(\frac{p q^{1 / 2}}{a}, \frac{p q^{1 / 2}}{b}, \frac{q^{1 / 2}}{c}, \frac{q^{1 / 2}}{d} ; p ; q\right) .
$$

Дуальная задача

$$
D^{*}(a, b, c, d ; p ; q) g(z ; v ; a, b ; \rho)=\lambda(v) D^{*}\left(a, b, c^{\prime}, d^{\prime} ; p ; q\right) g(z ; v ; a, b ; \rho)
$$

имеет решение

$$
g(z ; v ; a, b ; \rho)=\Gamma\left(a z^{ \pm 1}, b z^{ \pm 1}, \sqrt{\frac{\rho}{a b}} v^{ \pm 1} z^{ \pm 1} ; p, q\right),
$$

также определенное с точностью до умножения на произвольную функцию $\varphi(z), \varphi(q z)=\varphi(z)$. Теперь нетрудно заметить, что скалярное произведение 
решений (8.4) и (8.7) приводит к $V$-функции:

$$
\begin{aligned}
& \langle g(z ; v ; a, b ; e), f(z ; w ; c, d ; e)\rangle \\
& \quad=V\left(a, b, \sqrt{\frac{e}{a b}} v, \sqrt{\frac{e}{a b}} v^{-1} \frac{p q}{c}, \frac{p q}{d}, \sqrt{\frac{c d}{e}} w, \sqrt{\frac{c d}{e}} w^{-1} ; p, q\right) .
\end{aligned}
$$

Таким образом, эллиптический аналог гипергеометрической функции Гаусса оказывается напрямую связан с обобщенной спектральной задачей для линейной комбинации генераторов алгебры Склянина. Необходимо отметить, что наше скалярное произведение (8.5) отличается от инвариантной меры Склянина [82]. В [80], используя эту меру, Розенгрен построил интегральное представление для эллиптических $6 j$-символов и доказал гипотезу Склянина о воспроизводящем ядре для представлений в пространстве тэта-функций.

Неоднозначности в выборе функций $f$ и $g$ можно фиксировать требованием, чтобы эти функции удовлетворяли одновременно и уравнениям, полученным из (8.3) и (8.6) перестановкой $p$ и $q$ (так как равенства $\varphi(q z)=\varphi(p z)=\varphi(z)$ приводят к $\varphi(z)=$ const). Это означает, что мы вводим в рассмотрение вторую копию алгебры Склянина, полученную из первой перестановкой $\tau$ и $2 \eta$ :

$$
\widetilde{S}_{a}=i^{\delta_{a, 2}} \frac{\theta_{a+1}\left(\frac{\tau}{2} \mid 2 \eta\right)}{\theta_{1}(2 u \mid 2 \eta)}\left(\theta_{a+1}(2 u-2 g \mid 2 \eta) e^{\frac{\tau}{2} \partial_{u}}-\theta_{a+1}(-2 u-2 g \mid 2 \eta) e^{-\frac{\tau}{2} \partial_{u}}\right) .
$$

При этом для этих двух алгебр возникают следующие кросс-коммутационные соотношения:

$$
\begin{gathered}
S_{a} \widetilde{S}_{b}=\widetilde{S}_{b} S_{a}, \quad a, b \in\{0,3\} \quad \text { или } \quad a, b \in\{1,2\}, \\
S_{a} \widetilde{S}_{b}=-\widetilde{S}_{b} S_{a}, \quad a \in\{0,3\}, b \in\{1,2\} \quad \text { или } \quad a \in\{1,2\}, b \in\{0,3\} .
\end{gathered}
$$

Можно подставить в уравнения (8.3) и (8.6) параметризацию $z=e^{2 \pi i u / \omega_{2}}$, $2 \eta=\omega_{1} / \omega_{2}, \tau=\omega_{3} / \omega_{2}$ и построить их решения, корректно определенные при $|q|=1$, с помощью модифицированной эллиптической гамма-функции $G(u ; \boldsymbol{\omega})$. В этом случае однозначности решений можно добиться требованием, чтобы они одновременно удовлетворяли уравнениям, полученным из первоначальных перестановкой $\omega_{1}$ и $\omega_{2}$. Это приводит к другой копии алгебры Склянина, получающейся из первой заменами $\eta \rightarrow 1 /(4 \eta), u \rightarrow u /(2 \eta), \tau \rightarrow \tau /(2 \eta)$ :

$$
\widetilde{S}_{a}=i^{\delta_{a, 2}} \frac{\theta_{a+1}\left(\frac{1}{4 \eta} \mid \frac{\tau}{2 \eta}\right)}{\theta_{1}\left(\frac{u}{\eta} \mid \frac{\tau}{2 \eta}\right)}\left(\theta_{a+1}\left(\frac{u-g}{\eta} \mid \frac{\tau}{2 \eta}\right) e^{\frac{1}{2} \partial_{u}}-\theta_{a+1}\left(\frac{-u-g}{\eta} \mid \frac{\tau}{2 \eta}\right) e^{-\frac{1}{2} \partial_{u}}\right) .
$$

В этом случае часть генераторов $S_{a}$ и $\widetilde{S}_{a}$ также антикоммутируют друг с другом.

Описанные прямые произведения пар алгебр Склянина могут рассматриваться как эллиптические аналоги модулярного дубля Фаддеева $U_{q}\left(s l_{2}\right) \otimes$ $U_{\tilde{q}^{-1}}\left(s l_{2}\right)$ [17], [84]. Этот дубль можно получить из второго случая в пределе $\operatorname{Im}(\tau) \rightarrow+\infty$ (в первом случае такой предел не определен) [79]. 


\section{9. Разложения на простые дроби и определители}

В доказательствах многих тождеств для обычных и $q$-гипергеометрических рядов и интегралов используются разложения на простые дроби различных рациональных функций, заданных в виде отношений двух многочленов. На эллиптическом уровне эти функции заменяются на отношения произведений тэта-функций и ищутся разложения на суммы отношений тэта-функций с минимальным числом полюсов. Если разложение на простые дроби для произвольной рациональной функции - стандартная процедура, то в случае тэтафункций это не так. Первое такое известное соотношение связано с тождеством, приведенным в [85] в виде упражнения.

ТеОрема 7. Пусть $2 n$ переменных $a_{1}, \ldots, a_{n}, b_{1}, \ldots, b_{n} \in \mathbb{C}^{*}$ удовлетворяют ограничению $\prod_{k=1}^{n} a_{k}=\prod_{k=1}^{n} b_{k} u a_{j} / a_{k} \neq p^{k}, k \in \mathbb{Z}$, nрu $j \neq k$. Тогда справедливо следующее соотношение для тэта-функций:

$$
\sum_{k=1}^{n} \frac{\prod_{j=1}^{n} \theta\left(a_{k} / b_{j} ; p\right)}{\prod_{j=1, \neq k}^{n} \theta\left(a_{k} / a_{j} ; p\right)}=0
$$

ДокАЗАТЕЛЬСтво. Заменим в (9.1) $n$ на $n+1$, обозначим $a_{n+1}=t$ и подставим $b_{n+1}=a_{1} \cdots a_{n} t /\left(b_{1} \cdots b_{n}\right)$. Выделив $(n+1)$-й член из суммы, это соотношение можно переписать в виде [86]

$$
\prod_{k=1}^{n} \frac{\theta\left(t / b_{k} ; p\right)}{\theta\left(t / a_{k} ; p\right)}=\sum_{r=1}^{n} \frac{\theta\left(t a_{1} \cdots a_{n} /\left(a_{r} b_{1} \cdots b_{n}\right) ; p\right)}{\theta\left(t / a_{r}, a_{1} \cdots a_{n} /\left(b_{1} \cdots b_{n}\right) ; p\right)} \frac{\prod_{j=1}^{n} \theta\left(a_{r} / b_{j} ; p\right)}{\prod_{j=1, \neq r}^{n} \theta\left(a_{r} / a_{j} ; p\right)}
$$

и интерпретировать как разложение на простую дробь по тэта-функциям. Тогда доказательство этого тождества достаточно элементарно. При $n=2$ оно сводится к формуле сложения (А.5). По индукции следует, что левую часть можно разложить в сумму

$$
\sum_{r=1}^{n} c_{r} \frac{\theta\left(t a_{1} \cdots a_{n} /\left(a_{r} b_{1} \cdots b_{n}\right) ; p\right)}{\theta\left(t / a_{r} ; p\right)}
$$

коэффициенты $c_{r}$ в которой легко находятся умножением на $\theta\left(t / a_{r} ; p\right)$ и выбоpom $t=a_{r}$.

Теорема 8 [87]. Пусть $2 n$ переменных $a_{1}, \ldots, a_{n}, b_{1}, \ldots, b_{n} \in \mathbb{C}^{*}$ удовлетворяют соотношениям $a_{j} a_{k}, a_{j} / a_{k} \neq p^{k}, k \in \mathbb{Z}$, при $j \neq k$. Тогда справедливо следующее тождество для тэта-функиий:

$$
\sum_{k=1}^{n} \frac{a_{k} \prod_{j=1}^{n-2} \theta\left(a_{k} b_{j}^{ \pm 1} ; p\right)}{\prod_{j=1, \neq k}^{n} \theta\left(a_{k} a_{j}^{ \pm 1} ; p\right)}=0
$$

ДокАЗАТЕЛЬство. После замен $n \rightarrow n+1$ и $a_{n+1} \rightarrow t$ в $(9.3)$ и выделения $(n+1)$-го члена из суммы возникает разложение на простую дробь вида

$$
\frac{\prod_{j=1}^{n-1} \theta\left(t b_{j}^{ \pm 1} ; p\right)}{\prod_{j=1}^{n} \theta\left(t a_{j}^{ \pm 1} ; p\right)}=\sum_{k=1}^{n} \frac{\prod_{j=1}^{n-1} \theta\left(a_{k} b_{j}^{ \pm 1} ; p\right)}{\theta\left(t a_{k}^{ \pm 1} ; p\right) \prod_{j=1, \neq k}^{n} \theta\left(a_{k} a_{j}^{ \pm 1} ; p\right)},
$$

которое легко доказывается по рекурсии. 
Эти разложения на “простые” дроби для тэта-функций использовались в работах [33], [36], [86] для доказательства некоторых точных формул суммирования и интегрирования для эллиптических гипергеометрических функций. Приведем также другое разложение, использованное недавно в [59]:

$$
\frac{\prod_{j=1}^{n+2} \theta\left(v_{j} z ; p\right)}{z \theta\left(z^{2} ; p\right) \prod_{i=1}^{n} \theta\left(u_{i} z^{-1} ; p\right)}+\left(z \rightarrow z^{-1}\right)=\sum_{i=1}^{n} \frac{\prod_{j=1}^{n+2} \theta\left(u_{i} v_{j} ; p\right)}{u_{i} \theta\left(u_{i} z^{ \pm 1} ; p\right) \prod_{k=1, \neq i}^{n} \theta\left(u_{k} u_{i}^{-1} ; p\right)}
$$

где $\prod_{i=1}^{n} u_{i} \prod_{j=1}^{n+2} v_{j}=p^{n-1}$.

Эллиптический аналог определителя Коши имеет вид

$$
\begin{aligned}
\operatorname{det}_{1 \leqslant i, j \leqslant n} & \left(\frac{1}{a_{i}^{-1} \theta\left(a_{i} z_{j}^{ \pm 1} ; p\right)}\right) \\
= & (-1)^{n(n-1) / 2} \frac{\prod_{1 \leqslant i<j \leqslant n} a_{i}^{-1} \theta\left(a_{i} a_{j}^{ \pm 1} ; p\right) \prod_{1 \leqslant i<j \leqslant n} z_{i}^{-1} \theta\left(z_{i} z_{j}^{ \pm 1} ; p\right)}{\prod_{1 \leqslant i, j \leqslant n} a_{i}^{-1} \theta\left(a_{i} z_{j}^{ \pm 1} ; p\right)} .
\end{aligned}
$$

Определитель Фробениуса имеет вид

$$
\operatorname{det}_{1 \leqslant i, j \leqslant n}\left(\frac{\theta\left(t a_{i} b_{j} ; p\right)}{\theta\left(t, a_{i} b_{j} ; p\right)}\right)=\frac{\theta\left(t \prod_{i=1}^{n} a_{i} b_{i} ; p\right)}{\theta(t ; p)} \frac{\prod_{1 \leqslant i<j \leqslant n} a_{j} b_{j} \theta\left(a_{i} / a_{j}, b_{i} / b_{j} ; p\right)}{\prod_{1 \leqslant i, j \leqslant n} \theta\left(a_{i} b_{j} ; p\right)} .
$$

В [53] Варнаар предложил новый определитель для тэта-функций

$$
\begin{aligned}
\operatorname{det}_{1 \leqslant i, j \leqslant n} & \left(\frac{\theta\left(a x_{i}, a c / x_{i}\right)_{n-j}}{\theta\left(b x_{i}, b c / x_{i}\right)_{n-j}}\right) \\
= & a^{\left(\begin{array}{c}
n \\
2
\end{array}\right)} q^{\left(\begin{array}{c}
n \\
3
\end{array}\right)} \prod_{1 \leqslant i<j \leqslant n} x_{j} \theta\left(x_{i} x_{j}^{-1}, c x_{i}^{-1} x_{j}^{-1} ; p\right) \prod_{i=1}^{n} \frac{\theta\left(b / a, a b c q^{2 n-2 i}\right)_{i-1}}{\theta\left(b x_{i}, b c / x_{i}\right)_{n-1}},
\end{aligned}
$$

где $\theta(a)_{n}=\prod_{j=0}^{n-1} \theta\left(a q^{j} ; p\right)$. При $p \rightarrow 0$ он сводится к определителю Кратенталера [88].

Формулы (9.4)-(9.6) использовались в [11], [34], [53], [89]-[91] и ряде других работ как вспомогательные средства для доказательства необходимых эллиптических гипергеометрических тождеств. Разложения на простые дроби и определители эквивалентны друг другу до некоторой степени. Например, при разложении определителя (9.5) по последней строке и вычислении каждого члена по той же самой формуле (9.5) меньшей размерности $n-1$ возникает тождество, эквивалентное (9.2). Поэтому формула (9.5) следует из (9.2) по индукции по $n$, и наоборот. Подобным же образом, формула (9.4) эквивалентна (9.3) [91]. Приложения определителей на уровне $q$-гипергеометрических функций описаны, например, в [20]. В статье [92] Розенгрен и Шлоссер систематически рассмотрели определители тэта-функций на корневых системах (в частности, в ней приведен подробный список литературы на эту тему) и построили ряд новых точно вычисляемых случаев, которые мы пропускаем для краткости. Приложения эллиптических определителей к некоторым проблемам теории чисел, комбинаторного анализа и статистической механики описаны в [93], [94]. 


\section{0. Эллиптические бета-интегралы на корневых системах}

10.1. Интегралы для системы корней $C_{n}$. Известны два различных обобщения эллиптического бета-интеграла (3.1) на многократные интегралы для системы корней $C_{n}$ (или $B C_{n}$ ), предложенные ван Диеженом и автором в [32], [33]. Опишем сначала многопараметрический интеграл типа I.

Теорема 9. Пусть $z_{1}, \ldots, z_{n} \in \mathbb{T} u$ комплексные параметры $t_{1}, \ldots, t_{2 n+4}$ $u p, q$ удовлетворяют ограничениям $|p|,|q|,\left|t_{j}\right|<1 u \prod_{j=1}^{2 n+4} t_{j}=p q$. Тогда

$$
\begin{gathered}
\kappa_{n} \int_{\mathbb{T}^{n}} \prod_{1 \leqslant j<k \leqslant n} \frac{1}{\Gamma\left(z_{j}^{ \pm 1} z_{k}^{ \pm 1} ; p, q\right)} \prod_{j=1}^{n} \frac{\prod_{m=1}^{2 n+4} \Gamma\left(t_{m} z_{j}^{ \pm 1} ; p, q\right)}{\Gamma\left(z_{j}^{ \pm 2} ; p, q\right)} \frac{d z_{1}}{z_{1}} \cdots \frac{d z_{n}}{z_{n}} \\
=\prod_{1 \leqslant m<s \leqslant 2 n+4} \Gamma\left(t_{m} t_{s} ; p, q\right), \quad \kappa_{n}=\frac{(p ; p)_{\infty}^{n}(q ; q)_{\infty}^{n}}{(4 \pi i)^{n} n !} .
\end{gathered}
$$

ДокАЗАТЕЛЬство. Рассмотрим функцию

$$
\begin{aligned}
\rho\left(z, t ; C_{n}\right)= & \prod_{1 \leqslant i<j \leqslant n} \frac{1}{\Gamma\left(z_{i}^{ \pm 1} z_{j}^{ \pm 1} ; p, q\right)} \prod_{i=1}^{n} \frac{\prod_{m=1}^{2 n+3} \Gamma\left(t_{m} z_{i}^{ \pm 1} ; p, q\right)}{\Gamma\left(z_{i}^{ \pm 2}, A z_{i}^{ \pm 1} ; p, q\right)} \\
& \times \frac{\prod_{m=1}^{2 n+3} \Gamma\left(A t_{m}^{-1} ; p, q\right)}{\prod_{1 \leqslant m<s \leqslant 2 n+3} \Gamma\left(t_{m} t_{s} ; p, q\right)},
\end{aligned}
$$

где $A=\prod_{m=1}^{2 n+3} t_{m}$. Для всех $z_{i}$ в точках

$$
\mathscr{P}=\left\{t_{m} q^{a} p^{b}, A^{-1} q^{a+1} p^{b+1}\right\}_{m=1, \ldots, 2 n+3, a, b=0,1, \ldots},
$$

сходящихся к нулю, находятся полюсы функции (10.2). Координаты полюсов, уходящих в бесконечность, образуют множество $\mathscr{P}^{-1}$. Тогда утверждение теоремы можно переписать в виде

$$
\int_{C^{n}} \rho\left(z, t ; C_{n}\right) \frac{d z}{z}=\frac{(4 \pi i)^{n} n !}{(q ; q)_{\infty}^{n}(p ; p)_{\infty}^{n}}, \quad \frac{d z}{z}:=\prod_{j=1}^{n} \frac{d z_{j}}{z_{j}}
$$

где контур $C$ является произвольной деформацией $\mathbb{T}$, разделяющей $\mathscr{P}$ и $\mathscr{P}-1$.

Ядро интеграла $\rho\left(z, t ; C_{n}\right)$ удовлетворяет уравнению, аналогичному (3.4):

$$
\rho\left(z, q t_{1}, t_{2}, \ldots, t_{2 n+3} ; C_{n}\right)-\rho\left(z, t ; C_{n}\right)=\sum_{i=1}^{n}\left(g_{i}\left(z_{1}, \ldots, q^{-1} z_{i}, \ldots, z_{n}, t\right)-g_{i}(z, t)\right)
$$

где

$$
g_{i}(z, t)=\rho\left(z, t ; C_{n}\right) \prod_{j=1, \neq i}^{n} \frac{\theta\left(t_{1} z_{j}^{ \pm 1} ; p\right)}{\theta\left(z_{i} z_{j}^{ \pm 1} ; p\right)} \frac{\prod_{m=1}^{2 n+3} \theta\left(t_{m} z_{i} ; p\right)}{\prod_{m=2}^{2 n+3} \theta\left(t_{1} t_{m} ; p\right)} \frac{\theta\left(t_{1} A ; p\right)}{\theta\left(z_{i}^{2}, A z_{i} ; p\right)} \frac{t_{1}}{z_{i}}
$$


Разделив уравнение $(10.4)$ на $\rho\left(z, t ; C_{n}\right)$, мы получаем

$$
\begin{aligned}
& \prod_{i=1}^{n} \frac{\theta\left(t_{1} z_{i}^{ \pm 1} ; p\right)}{\theta\left(A z_{i}^{ \pm 1} ; p\right)} \prod_{m=2}^{2 n+3} \frac{\theta\left(A t_{m}^{-1} ; p\right)}{\theta\left(t_{1} t_{m} ; p\right)}-1=\frac{t_{1} \theta\left(t_{1} A ; p\right)}{\prod_{m=2}^{2 n+3} \theta\left(t_{1} t_{m} ; p\right)} \sum_{i=1}^{n} \frac{1}{z_{i} \theta\left(z_{i}^{2} ; p\right)} \\
& \quad \times \prod_{j=1, \neq i}^{n} \frac{\theta\left(t_{1} z_{j}^{ \pm 1} ; p\right)}{\theta\left(z_{i} z_{j}^{ \pm 1} ; p\right)}\left(z_{i}^{2 n+2} \frac{\prod_{m=1}^{2 n+3} \theta\left(t_{m} z_{i}^{-1} ; p\right)}{\theta\left(A z_{i}^{-1} ; p\right)}-\frac{\prod_{m=1}^{2 n+3} \theta\left(t_{m} z_{i} ; p\right)}{\theta\left(A z_{i} ; p\right)}\right) .
\end{aligned}
$$

Обе части этого равенства инвариантны относительно преобразования $z_{1} \rightarrow p z_{1}$ и имеют одинаковые множества полюсов (особенности в точках $z_{1}=z_{j}, z_{j}^{-1}$, $j=2, \ldots, n$, и $z_{1}= \pm p^{k / 2}, k \in \mathbb{Z}$, в правой части сокращаются) и вычетов в них. Поэтому функции в обеих частях равенства (10.6) отличаются только аддитивной постоянной, не зависящей от $z_{1}$. Эта постоянная равна нулю, что следует из тривиальной проверки равенства (10.6) при $z_{1}=t_{1}$.

Интегрируя (10.4) по переменным $z \in C^{n}$, получаем

$$
I\left(q t_{1}, t_{2}, \ldots, t_{2 n+3}\right)-I(t)=\sum_{i=1}^{n}\left(\int_{C^{i-1} \times\left(q^{-1} C\right) \times C^{n-i}}-\int_{C^{n}}\right) g_{i}(z, t) \frac{d z}{z},
$$

где $I(t)=\int_{C^{n}} \rho\left(z, t ; C_{n}\right) \frac{d z}{z}$ и $q^{-1} C$ обозначает контур $C$, растянутый относительно нулевой точки.

Полюсы функции (10.5) по переменным $z_{i}$ сходятся к нулю по точкам $z_{i}=$ $t_{m} q^{a} p^{b}, A^{-1} q^{a} p^{b+1}$ и уходят на бесконечность при $z_{i}=t_{m}^{-1} q^{-1-a} p^{-b}, A q^{-a} p^{-b-1}$, где $m=1, \ldots, 2 n+3, a, b=0,1,2, \ldots$. При $\left|t_{m}\right|<1$ и $|p|<|A|$ область $1 \leqslant$ $\left|z_{i}\right| \leqslant|q|^{-1}$ не содержит полюсов, так что можно положить $C=\mathbb{T}$, деформировать $q^{-1} \mathbb{T}$ в $\mathbb{T}$ в $(10.7)$ и получить равенство $I\left(q t_{1}, t_{2}, \ldots, t_{2 n+3}\right)=I(t)$.

Повторяя почти дословно процедуру аналитического продолжения, использовавшуюся в случае $n=1$, получаем, что $I$ есть постоянная, зависящая только от $p$ и $q$. Ее значение находится переходом к пределам $t_{j} t_{j+n} \rightarrow 1, j=1, \ldots, n$, аналогично случаю $n=1$, что дает правую часть (10.3).

Формула (10.1) предложена и частично обоснована в [33] и полностью доказана различными способами в [34], [43], [59]. В специальном пределе $p \rightarrow 0$ она сводится к одной из формул интегрирования Густафсона [95].

Система корней $C_{n}$ состоит из множества векторов из $\mathbb{R}^{n}$ вида $X\left(C_{n}\right)=$ $\left.\left\{ \pm 2 e_{i}, \pm e_{i} \pm e_{j}, i<j\right\}\right|_{i, j=1, \ldots, n}$, где $e_{i}$ - ортонормированный базис $\mathbb{R}^{n}$. Обозначая $z_{i}=\exp \left(e_{i}\right)$, мы видим, что знаменатель ядра интеграла (10.1) содержит произведение по корням $C_{n}$ вида

$$
\prod_{\alpha \in X\left(C_{n}\right)} \Gamma\left(\mathrm{e}^{\alpha} ; p, q\right)=\prod_{i=1}^{n} \Gamma\left(z_{i}^{ \pm 2} ; p, q\right) \prod_{1 \leqslant i<j \leqslant n} \Gamma\left(z_{i}^{ \pm 1} z_{j}^{ \pm 1} ; p, q\right) .
$$

Система корней $B C_{n}$ содержит дополнительно векторы $\left\{ \pm e_{1}, \ldots, \pm e_{n}\right\}$, но общие закономерности появления этих векторов в ядрах интегралов еще не установлены. Группа Вейля этих систем $S_{n} \times \mathbb{Z}_{2}^{n}$ является симметрией ядра интеграла.

Эллиптический $C_{n}$-бета-интеграл типа II строится с помощью формулы (10.1) чисто алгебраическим способом [33]. 
Теорема 10. Пусть комплексные параметры $t, t_{m}(m=1, \ldots, 6), p$ u $q$ удовлетворяют условиями $|p|,|q|,|t|,\left|t_{m}\right|<1$ u $t^{2 n-2} \prod_{m=1}^{6} t_{m}=p q$. Тогда

$$
\begin{gathered}
\kappa_{n} \int_{\mathbb{T}^{n}} \prod_{1 \leqslant j<k \leqslant n} \frac{\Gamma\left(t z_{j}^{ \pm 1} z_{k}^{ \pm 1} ; p, q\right)}{\Gamma\left(z_{j}^{ \pm 1} z_{k}^{ \pm 1} ; p, q\right)} \prod_{j=1}^{n} \frac{\prod_{m=1}^{6} \Gamma\left(t_{m} z_{j}^{ \pm 1} ; p, q\right)}{\Gamma\left(z_{j}^{ \pm 2} ; p, q\right)} \frac{d z_{1}}{z_{1}} \cdots \frac{d z_{n}}{z_{n}} \\
=\prod_{j=1}^{n}\left(\frac{\Gamma\left(t^{j} ; p, q\right)}{\Gamma(t ; p, q)} \prod_{1 \leqslant m<s \leqslant 6} \Gamma\left(t^{j-1} t_{m} t_{s} ; p, q\right)\right) .
\end{gathered}
$$

ДокАЗАТЕЛЬСтво. Обозначим интеграл в левой части (10.8) через $I_{n}(t$, $\left.t_{1}, \ldots, t_{5}\right)$ и рассмотрим $(2 n-1)$-кратный интеграл

$$
\begin{aligned}
\kappa_{n} \kappa_{n-1} & \int_{\mathbb{T}^{2 n-1}} \prod_{\substack{1 \leqslant j<k \leqslant n \\
\Gamma\left(z_{j}^{ \pm 1} z_{k}^{ \pm 1} ; p, q\right)}} \prod_{j=1}^{n} \frac{\prod_{r=0}^{5} \Gamma\left(t_{r} z_{j}^{ \pm 1} ; p, q\right)}{\Gamma\left(z_{j}^{ \pm 2} ; p, q\right)} \\
\times & \prod_{\substack{1 \leqslant j \leqslant n \\
1 \leqslant k \leqslant n-1}} \Gamma\left(t^{1 / 2} z_{j}^{ \pm 1} w_{k}^{ \pm 1} ; p, q\right) \prod_{1 \leqslant j<k \leqslant n-1} \frac{1}{\Gamma\left(w_{j}^{ \pm 1} w_{k}^{ \pm 1} ; p, q\right)} \\
& \times \prod_{j=1}^{n-1} \frac{\Gamma\left(w_{j}^{ \pm 1} t^{n-3 / 2} \prod_{s=1}^{5} t_{s} ; p, q\right)}{\Gamma\left(w_{j}^{ \pm 2}, w_{j}^{ \pm 1} t^{2 n-3 / 2} \prod_{s=1}^{5} t_{s} ; p, q\right)} \frac{d w_{1}}{w_{1}} \cdots \frac{d w_{n-1}}{w_{n-1}} \frac{d z_{1}}{z_{1}} \cdots \frac{d z_{n}}{z_{n}}
\end{aligned}
$$

с $p, q, t$ и $t_{r}, r=0, \ldots, 5$, лежащими внутри единичного круга, такими, что $t^{n-1} \prod_{r=0}^{5} t_{r}=p q$. Интегрирование по переменным $w_{j}$ с помощью формулы (10.1) приводит выражение (10.9) к виду $\Gamma^{n}(t ; p, q) \Gamma^{-1}\left(t^{n} ; p, q\right) I_{n}\left(t, t_{1}, \ldots, t_{5}\right)$ (в котором подразумевается, что $t_{6}=p q t^{2-2 n} / \prod_{j=1}^{5} t_{j}$ ). В силу ограниченности подынтегральной функции на контуре интегрирования можно поменять порядок интегрирования. Тогда интегрирование по $z_{k}$-переменным с помощью формулы (10.1) переводит выражение (10.9) в

$$
\Gamma^{n-1}(t ; p, q) \prod_{0 \leqslant r<s \leqslant 5} \Gamma\left(t_{r} t_{s} ; p, q\right) I_{n-1}\left(t, t^{1 / 2} t_{1}, \ldots, t^{1 / 2} t_{5}\right) .
$$

В результате возникает рекуррентное соотношение, связывающее интегралы разных размерностей $n$ :

$$
I_{n}\left(t, t_{1}, \ldots, t_{5}\right)=\frac{\Gamma\left(t^{n} ; p, q\right)}{\Gamma(t ; p, q)} \prod_{0 \leqslant r<s \leqslant 5} \Gamma\left(t_{r} t_{s} ; p, q\right) I_{n-1}\left(t, t^{1 / 2} t_{1}, \ldots, t^{1 / 2} t_{5}\right) .
$$

Пользуясь известным начальным условием (3.1) при $n=1$, из рекурсии находим (10.8).

Если выразить $t_{6}$ через другие параметры и устранить множители $p q$ в аргументах эллиптических гамма-функций с помощью формулы отражения для $\Gamma(z ; p, q)$, то легко перейти к пределу $p \rightarrow 0$ при фиксированных параметрах. Это приводит к многократному q-бета-интегралу Густафсона [96]. Ряд дальнейших предельных переходов по параметрам приводит к интегралу Сельбер- 
га - фундаментально важному интегралу, имеющему большое количество приложений в математической физике [97]:

$$
\begin{gathered}
\int_{0}^{1} \cdots \int_{0}^{1} \prod_{1 \leqslant j \leqslant n} x_{j}^{\alpha-1}\left(1-x_{j}\right)^{\beta-1} \prod_{1 \leqslant j<k \leqslant n}\left|x_{j}-x_{k}\right|^{2 \gamma} d x_{1} \cdots d x_{n} \\
=\prod_{1 \leqslant j \leqslant n} \frac{\Gamma(\alpha+(j-1) \gamma) \Gamma(\beta+(j-1) \gamma) \Gamma(1+j \gamma)}{\Gamma(\alpha+\beta+(n+j-2) \gamma) \Gamma(1+\gamma)},
\end{gathered}
$$

где $\operatorname{Re}(\alpha), \operatorname{Re}(\beta)>0$ и $\operatorname{Re}(\gamma)>-\min (1 / n, \operatorname{Re}(\alpha) /(n-1), \operatorname{Re}(\beta) /(n-1))$.

Поэтому формула (10.8) представляет собой эллиптический аналог интеграла Сельберга. Она может также интерпретироваться как эллиптическое обобщение $B C_{n}$-тождеств Макдональда-Морриса для постоянных членов [98]. Приведенное доказательство заимствовано из работы [33]. Оно моделирует доказательство интеграла Сельберга, предложенное Андерсоном [99], и представляет собой обобщение метода, использованного Густафсоном в [96] для доказательства соответствующего q-бета-интеграла.

10.2. Интегралы для системы корней $A_{n}$. Различные эллиптические бета-интегралы на корневой системе $A_{n}$ были предложены в работах [11], [36]. По аналогии с $C_{n}$-случаями формулы интегрирования, зависящие от $2 n+3$ параметров, будем относить к типу I. Точно вычисляемые интегралы с меньшим числом параметров, которые выводятся с помощью интегралов типа I, будем относить к типу II. Перечислим эти формулы, опуская их доказательства.

ТЕОРема 11. Пусть $|p|,|q|<1 u 2 n+4$ параметра $t_{m}, s_{m}, m=1, \ldots, n+2$, удовлетворяют ограничениям $\left|t_{m}\right|,\left|s_{m}\right|<1 u S T=p q$, где $S=\prod_{m=1}^{n+2} s_{m}$ u $T=\prod_{m=1}^{n+2} t_{m}$. Тогда

$$
\begin{aligned}
\kappa_{n}^{A} \int_{\mathbb{T}^{n}} & \prod_{1 \leqslant j<k \leqslant n+1} \frac{1}{\Gamma\left(z_{i} z_{j}^{-1}, z_{i}^{-1} z_{j} ; p, q\right)} \prod_{j=1}^{n+1} \prod_{m=1}^{n+2} \Gamma\left(s_{m} z_{j}, t_{m} z_{j}^{-1} ; p, q\right) \frac{d z}{z} \\
\quad= & \prod_{m=1}^{n+2} \Gamma\left(S s_{m}^{-1}, T t_{m}^{-1} ; p, q\right) \prod_{k, m=1}^{n+2} \Gamma\left(s_{k} t_{m} ; p, q\right)
\end{aligned}
$$

где $z_{1} z_{2} \cdots z_{n+1}=1 u$

$$
\kappa_{n}^{A}=\frac{(p ; p)_{\infty}^{n}(q ; q)_{\infty}^{n}}{(2 \pi i)^{n}(n+1) !} .
$$

В этом интеграле типа I мы имеем разделение $2 n+4$ параметров (однородных в $C_{n}$-случае) с фиксированным произведением на две однородные группы по $n+2$ элемента. Эта формула интегрирования была предложена и частично обоснована в [11], различные полные доказательства даны в [34], [43]. В простейшем пределе $p \rightarrow 0$ возникает один из интегралов Густафсона [95].

Система корней $A_{n}$ состоит из векторов $X\left(A_{n}\right)=\left.\left\{e_{i}-e_{j}, i \neq j\right\}\right|_{i, j=1, \ldots, n+1}$, где $e_{i}$ - ортонормированный базис $\mathbb{R}^{n+1}$. Эти векторы лежат в гиперповерхности, ортогональной вектору $E=\sum_{i=1}^{n+1} e_{i}$. Полагая $z_{i}=\exp \left(e_{i}-E /(n+1)\right)$, 
получаем $z_{1} \cdots z_{n+1}=1$. Знаменатель ядра интеграла (10.11) содержит произведение вида

$$
\prod_{\alpha \in X\left(A_{n}\right)} \Gamma\left(\mathrm{e}^{\alpha} ; p, q\right)=\prod_{1 \leqslant i<j \leqslant n+1} \Gamma\left(\frac{z_{i}}{z_{j}}, \frac{z_{j}}{z_{i}} ; p, q\right) .
$$

Полное ядро интеграла инвариантно относительно $A_{n}$-группы Вейля $S_{n+1}$.

Существует дополнительный независимый $A_{n}$-интеграл типа I [36].

Теорема 12. Пусть $|p|,|q|<1$ u $2 n+3$ параметра $t_{k}, k=1, \ldots, n, u s_{m}$, $m=1, \ldots, n+3$, удовлетворяют ограничениям $\left|t_{k}\right|<1,\left|s_{m}\right|<1 u|p q|<\left|S t_{k}\right|$, где $S=\prod_{m=1}^{n+3} s_{m}$. Тогда справедлива следующая явная формула интегрирования:

$$
\begin{aligned}
\kappa_{n}^{A} \int_{\mathbb{T}^{n}} & \prod_{1 \leqslant i<j \leqslant n+1} \frac{\Gamma\left(S z_{i}^{-1} z_{j}^{-1} ; p, q\right)}{\Gamma\left(z_{i} z_{j}^{-1}, z_{i}^{-1} z_{j} ; p, q\right)} \\
& \times \prod_{j=1}^{n+1} \frac{\prod_{k=1}^{n} \Gamma\left(t_{k} z_{j} ; p, q\right) \prod_{m=1}^{n+3} \Gamma\left(s_{m} z_{j}^{-1} ; p, q\right)}{\prod_{k=1}^{n} \Gamma\left(S t_{k} z_{j}^{-1} ; p, q\right)} \frac{d z}{z} \\
= & \prod_{k=1}^{n} \prod_{m=1}^{n+3} \frac{\Gamma\left(t_{k} s_{m} ; p, q\right)}{\Gamma\left(S t_{k} s_{m}^{-1} ; p, q\right)} \prod_{1 \leqslant l<m \leqslant n+3} \Gamma\left(S s_{l}^{-1} s_{m}^{-1} ; p, q\right),
\end{aligned}
$$

где $z_{1} \cdots z_{n+1}=1$.

Здесь мы имеем разделение $2 n+3$ независимых параметров на две однородные группы с $n$ и $n+3$ элементами. В пределе $p \rightarrow 0$ возникает $q$-бета-интеграл, который не рассматривался в литературе до вывода эллиптического случая, так же, как и его чисто гипергеометрическое вырождение, получающееся в пределе $q \rightarrow 1$.

Имеется несколько $A_{n}$-эллиптических бета-интегралов типа II [11]. Обозначим

$$
\begin{aligned}
I^{\mathrm{II}}\left(\mathbf{t}, \mathbf{s} ; p, q ; A_{n}\right)=\kappa_{n}^{A} & \int_{\mathbb{T}^{n}} \prod_{1 \leqslant i<j \leqslant n+1} \frac{\Gamma\left(t z_{i} z_{j} ; p, q\right)}{\Gamma\left(z_{i} z_{j}^{-1}, z_{i}^{-1} z_{j} ; p, q\right)} \\
& \times \prod_{j=1}^{n+1} \prod_{k=1}^{n+1} \Gamma\left(t_{k} z_{j}^{-1} ; p, q\right) \prod_{i=1}^{4} \Gamma\left(s_{i} z_{j} ; p, q\right) \frac{d z_{1}}{z_{1}} \cdots \frac{d z_{n}}{z_{n}},
\end{aligned}
$$

где $\left|t_{k}\right|,\left|s_{i}\right|<1, k=1, \ldots, n+1, i=1, \ldots, 4$, и

$$
t^{n-1} \prod_{k=1}^{n+1} t_{k} \prod_{i=1}^{4} s_{i}=p q, \quad \prod_{j=1}^{n+1} z_{j}=1 .
$$

ТеОрема 13. При указанных ограничениях на параметры справедливы следующие $A_{n}$-бормулы интегрирования. Для нечетных $n$ имеем

$$
\begin{aligned}
I^{\mathrm{II}}\left(\mathbf{t}, \mathbf{s} ; p, q ; A_{n}\right)= & \prod_{1 \leqslant j<k \leqslant n+1} \Gamma\left(t t_{j} t_{k} ; p, q\right) \prod_{k=1}^{n+1} \prod_{i=1}^{4} \Gamma\left(t_{k} s_{i} ; p, q\right) \\
& \times \frac{\Gamma\left(t^{\frac{n+1}{2}}, A ; p, q\right)}{\Gamma\left(t^{\frac{n+1}{2}} A ; p, q\right)} \prod_{1 \leqslant i<m \leqslant 4} \Gamma\left(t^{\frac{n-1}{2}} s_{i} s_{m} ; p, q\right),
\end{aligned}
$$


где $A=\prod_{k=1}^{n+1} t_{k} ;$ для четных $n$

$$
\begin{aligned}
I^{\mathrm{II}}\left(\mathbf{t}, \mathbf{s} ; p, q ; A_{n}\right)= & \prod_{1 \leqslant j<k \leqslant n+1} \Gamma\left(t t_{j} t_{k} ; p, q\right) \prod_{k=1}^{n+1} \prod_{i=1}^{4} \Gamma\left(t_{k} s_{i} ; p, q\right) \\
& \times \Gamma(A ; p, q) \prod_{i=1}^{4} \frac{\Gamma\left(t^{\frac{n}{2}} s_{i} ; p, q\right)}{\Gamma\left(t^{\frac{n}{2}} A s_{i} ; p, q\right)} .
\end{aligned}
$$

Эти формулы содержат только $n+5$ свободных параметров. Они могут быть выведены непосредственно из эллиптических бета-интегралов типа I (10.1) и (10.11) [11]. В простейшем пределе $p \rightarrow 0$ они сводятся к $q$-бета-интегралам Густафсона-Ракха [100].

Для описания другого $A_{n}$-интеграла типа II необходимы десять комплексных параметров $p, q, t, s, t_{1}, t_{2}, t_{3}, s_{1}, s_{2}, s_{3}$ с ограничениями $(t s)^{n-1} \prod_{k=1}^{3} t_{k} s_{k}=p q$ и $|p|,|q|,|t|,|s|,\left|t_{k}\right|,\left|s_{k}\right|<1$. Определим теперь интеграл

$$
\begin{aligned}
& I^{\mathrm{II}}\left(\mathbf{t}, \mathbf{s} ; p, q ; A_{n}\right) \\
& \quad=\kappa_{n}^{A} \int_{\mathbb{T}^{n}} \prod_{1 \leqslant i<j \leqslant n+1} \frac{\Gamma\left(t z_{i} z_{j}, s z_{i}^{-1} z_{j}^{-1} ; p, q\right)}{\Gamma\left(z_{i} z_{j}^{-1}, z_{i}^{-1} z_{j} ; p, q\right)} \prod_{j=1}^{n+1} \prod_{k=1}^{3} \Gamma\left(t_{k} z_{j}, s_{k} z_{j}^{-1} ; p, q\right) \frac{d z}{z} .
\end{aligned}
$$

ТеОрема 14. При указанных ограничениях на параметры справедливы следующие формулы интегрирования для корневой системы $A_{n}$. Для нечетных $n$ въполнено

$$
\begin{aligned}
I^{\mathrm{II}}(\mathbf{t}, \mathbf{s} ; p, q ; & \left.A_{n}\right)=\Gamma\left(t^{\frac{n+1}{2}}, s^{\frac{n+1}{2}} ; p, q\right) \prod_{1 \leqslant i<k \leqslant 3} \Gamma\left(t^{\frac{n-1}{2}} t_{i} t_{k}, s^{\frac{n-1}{2}} s_{i} s_{k} ; p, q\right) \\
& \times \prod_{j=1}^{(n+1) / 2} \prod_{i, k=1}^{3} \Gamma\left((t s)^{j-1} t_{i} s_{k} ; p, q\right) \\
& \times \prod_{j=1}^{(n-1) / 2}\left(\Gamma\left((t s)^{j} ; p, q\right) \prod_{1 \leqslant i<k \leqslant 3} \Gamma\left(t^{j-1} s^{j} t_{i} t_{k}, t^{j} s^{j-1} s_{i} s_{k} ; p, q\right)\right)
\end{aligned}
$$

для четных $n$

$$
\begin{aligned}
I^{\mathrm{II}}\left(\mathbf{t}, \mathbf{s} ; p, q ; A_{n}\right)=\prod_{i=1}^{3} & \Gamma\left(t^{\frac{n}{2}} t_{i}, s^{\frac{n}{2}} s_{i} ; p, q\right) \Gamma\left(t^{\frac{n}{2}-1} t_{1} t_{2} t_{3}, s^{\frac{n}{2}-1} s_{1} s_{2} s_{3} ; p, q\right) \\
& \times \prod_{j=1}^{n / 2}\left(\Gamma\left((t s)^{j} ; p, q\right) \prod_{i, k=1}^{3} \Gamma\left((t s)^{j-1} t_{i} s_{k} ; p, q\right)\right. \\
& \left.\times \prod_{1 \leqslant i<k \leqslant 3} \Gamma\left(t^{j-1} s^{j} t_{i} t_{k}, t^{j} s^{j-1} s_{i} s_{k} ; p, q\right)\right) .
\end{aligned}
$$

Эти формулы могут быть выведены непосредственно из $C_{n}$-эллиптических бета-интегралов типа I и II и $A_{n}$-интеграла типа I (10.11) [11]. В простейшем пределе $p \rightarrow 0$ они сводятся к $q$-гипергеометрическим интегралам Густафсона [96]. 


\section{1. Некоторые формулы суммирования многократных рядов}

Известно несколько формул суммирования многократных эллиптических гипергеометрических рядов, представляющих собой многомерные аналоги суммы Френкеля-Тураева [11], [34], [53], [86], [90], [101], [102]. Для некоторых из них была установлена прямая связь с суммами вычетов полюсов ядер соответствующих эллиптических бета-интегралов на корневых системах. Опишем некоторые из этих формул без доказательств.

ТеОрема 15. Пусть $|p|<1$ и параметр $q \in \mathbb{C}$ не равен целой степени $p$. Тогда имеет место формула суммирования

$$
\begin{aligned}
& \sum_{\substack{0 \leqslant \lambda_{j} \leqslant N_{j} \\
j=1, \ldots, n}} q^{\sum_{j=1}^{n} j \lambda_{j}} \prod_{1 \leqslant j<k \leqslant n} \frac{\theta\left(t_{j} t_{k} q^{\lambda_{j}+\lambda_{k}}, t_{j} t_{k}^{-1} q^{\lambda_{j}-\lambda_{k}} ; p\right)}{\theta\left(t_{j} t_{k}, t_{j} t_{k}^{-1} ; p\right)} \\
& \quad \times \prod_{1 \leqslant j \leqslant n}\left(\frac{\theta\left(t_{j}^{2} q^{2 \lambda_{j}} ; p\right)}{\theta\left(t_{j}^{2} ; p\right)} \prod_{0 \leqslant r \leqslant 2 n+3} \frac{\theta\left(t_{j} t_{r}\right)_{\lambda_{j}}}{\theta\left(q t_{j} t_{r}^{-1}\right)_{\lambda_{j}}}\right) \\
&=\theta\left(q a^{-1} b^{-1}, q a^{-1} c^{-1}, q b^{-1} c^{-1}\right)_{N_{1}+\cdots+N_{n}} \\
& \times \prod_{1 \leqslant j<k \leqslant n} \frac{\theta\left(q t_{j} t_{k}\right)_{N_{j}} \theta\left(q t_{j} t_{k}\right)_{N_{k}}}{\theta\left(q t_{j} t_{k}\right)_{N_{j}+N_{k}}} \\
& \times \prod_{1 \leqslant j \leqslant n} \frac{\theta\left(q t_{j}^{2}\right)_{N_{j}}}{\theta\left(q t_{j} a^{-1}, q t_{j} b^{-1}, q t_{j} c^{-1}, q^{1+N_{1}+\cdots+N_{n}-N_{j}} t_{j}^{-1} a^{-1} b^{-1} c^{-1}\right)_{N_{j}}}
\end{aligned}
$$

где $\theta(a)_{\lambda}=\Gamma\left(a q^{\lambda} ; p, q\right) / \Gamma(a ; p, q) u$

$$
\begin{aligned}
& q^{-1} \prod_{r=0}^{2 n+3} t_{r}=1 \quad \text { (условие балансировки), } \\
& q^{N_{j}} t_{j} t_{n+j}=1, \quad j=1, \ldots, n \quad \text { (условие обрьва), }
\end{aligned}
$$

$c N_{j}=0,1,2, \ldots, j=1, \ldots, n, u a=t_{2 n+1}, b=t_{2 n+2}, c=t_{2 n+3}$.

Эта формула выведена в [101] из $C_{n}$-эллиптического бета-интеграла типа I (еще недоказанного на тот момент). Ее первое рекурсивное доказательство получено в [86]. В пределе $p \rightarrow 0$ она вырождается в многомерную $8 \varphi_{7}$-сумму, найденную в [103].

Теорема 16. Пусть $N=0,1,2, \ldots$ и параметры $p, q, t, t_{0}, \ldots, t_{5} \in \mathbb{C}$ удовлетворяют ограничениям $|p|<1$ u

$$
\begin{aligned}
q^{-1} t^{2 n-2} \prod_{r=0}^{5} t_{r} & =1 \quad & (\text { условие балансировки }), \\
q^{N} t^{n-1} t_{0} t_{4} & =1 \quad & (\text { условие обрыва). }
\end{aligned}
$$


Тогда имеет место формула суммирования для многократного эллиптического гипергеометрического ряда

$$
\begin{aligned}
\sum_{0 \leqslant \lambda_{1} \leqslant \cdots \leqslant \lambda_{n} \leqslant N} & q^{\sum_{j=1}^{m} \lambda_{j}} t^{2 \sum_{j=1}^{m}(n-j) \lambda_{j}} \prod_{1 \leqslant j<k \leqslant m}\left(\frac{\theta\left(\tau_{k} \tau_{j} q^{\lambda_{k}+\lambda_{j}}, \tau_{k} \tau_{j}^{-1} q^{\lambda_{k}-\lambda_{j}} ; p\right)}{\theta\left(\tau_{k} \tau_{j}, \tau_{k} \tau_{j}^{-1} ; p\right)}\right. \\
& \left.\times \frac{\theta\left(t \tau_{k} \tau_{j}\right)_{\lambda_{k}+\lambda_{j}}}{\theta\left(q t^{-1} \tau_{k} \tau_{j}\right)_{\lambda_{k}+\lambda_{j}}} \frac{\theta\left(t \tau_{k} \tau_{j}^{-1}\right)_{\lambda_{k}-\lambda_{j}}}{\theta\left(q t^{-1} \tau_{k} \tau_{j}^{-1}\right)_{\lambda_{k}-\lambda_{j}}}\right) \\
& \times \prod_{j=1}^{m}\left(\frac{\theta\left(\tau_{j}^{2} q^{2 \lambda_{j}} ; p\right)}{\theta\left(\tau_{j}^{2} ; p\right)} \prod_{r=0}^{5} \frac{\theta\left(t_{r} \tau_{j}\right)_{\lambda_{j}}}{\theta\left(q t_{r}^{-1} \tau_{j}\right)_{\lambda_{j}}}\right) \\
= & \prod_{j=1}^{n} \frac{\theta\left(q t^{n+j-2} t_{0}^{2}\right)_{N} \prod_{1 \leqslant r<s \leqslant 3} \theta\left(q t^{1-j} t_{r}^{-1} t_{s}^{-1}\right)_{N}}{\theta\left(q t^{2-n-j} \prod_{r=0}^{3} t_{r}^{-1}\right)_{N} \prod_{r=1}^{3} \theta\left(q t^{j-1} t_{0} t_{r}^{-1}\right)_{N}}
\end{aligned}
$$

Здесъ используются обозначения $\tau_{j}=t_{0} t^{j-1}, j=1, \ldots, n$.

Эта формула суммирования была предложена Варнааром [53]. Она следует из $C_{n}$-эллиптического бета-интеграла типа II [32] и впервые была доказана рекурсивно в работе [45].

Для системы корней $A_{n}$ эллиптическая гипергеометрическая сумма типа I имеет вид

$$
\begin{aligned}
& \sum_{\substack{0 \leqslant \lambda_{j} \leqslant N_{j} \\
j=1, \ldots, n}} q^{\sum_{j=1}^{n} j \lambda_{j}} \prod_{j=1}^{n} \frac{\theta\left(t_{j} q^{\lambda_{j}+|\lambda|} ; p\right)}{\theta\left(t_{j} ; p\right)} \prod_{1 \leqslant i<j \leqslant n} \frac{\theta\left(t_{i} t_{j}^{-1} q^{\lambda_{i}-\lambda_{j}} ; p\right)}{\theta\left(t_{i} t_{j}^{-1} ; p\right)} \\
& \quad \times \prod_{i, j=1}^{n} \frac{\theta\left(t_{i} t_{j}^{-1} q^{-N_{j}}\right)_{\lambda_{i}}}{\theta\left(q t_{i} t_{j}^{-1}\right)_{\lambda_{i}}} \prod_{j=1}^{n} \frac{\theta\left(t_{j}\right)_{|\lambda|}}{\theta\left(t_{j} q^{1+N_{j}}\right)_{|\lambda|}} \frac{\theta(b, c)_{|\lambda|}}{\theta(q / d, q / e)_{|\lambda|}} \prod_{j=1}^{n} \frac{\theta\left(d t_{j}, e t_{j}\right)_{\lambda_{j}}}{\theta\left(t_{j} q / b, t_{j} q / c\right)_{\lambda_{j}}} \\
& =\frac{\theta(q /(b d), q /(c d))_{|N|}}{\theta(q / d, q /(b c d))_{|N|}} \prod_{j=1}^{n} \frac{\theta\left(t_{j} q, t_{j} q /(b c)\right)_{N_{j}}}{\theta\left(t_{j} q / b, t_{j} q / c\right)_{N_{j}}}
\end{aligned}
$$

где

$$
|\lambda|=\lambda_{1}+\cdots+\lambda_{n}, \quad|N|=N_{1}+\cdots+N_{n}, \quad b c d e=q^{1+|N|} .
$$

Эта формула доказана рекурсивно в [86]. Она вытекает также из анализа вычетов для интеграла типа I (10.11) [11] и при $p \rightarrow 0$ редуцируется в многократную ${ }_{8} \varphi_{7}$-сумму Милна [104].

Опуская ряд других установленных формул суммирования многократных эллиптических гипергеометрических рядов, приведем гипотезу из [11].

ГиПотезА. Пусть $|p|<1, N=0,1,2, \ldots$ u $n$ параметров $t_{k} \in \mathbb{C}, k=$ $1, \ldots, n$, удовлетворяют условию балансировки

$$
\prod_{k=1}^{n} t_{k}=q^{-N}
$$


Тогда справедлива следующая формула суммирования:

$$
\begin{aligned}
& \sum_{\substack{\lambda_{k}=0, \ldots, N \\
\lambda_{1}+\cdots+\lambda_{n}=N}} \frac{\prod_{1 \leqslant i<j \leqslant n} \theta\left(t t_{i} t_{j}\right)_{\lambda_{i}+\lambda_{j}} \prod_{i=1}^{n} \prod_{j=n+1}^{n+3} \theta\left(t t_{i} t_{j}\right)_{\lambda_{i}} \prod_{i, j=1}^{n} \theta\left(t_{i} t_{j}^{-1}\right)_{-\lambda_{j}}}{\prod_{i, j=1 ; i \neq j}^{n} \theta\left(t_{i} t_{j}^{-1}\right)_{\lambda_{i}-\lambda_{j}} \prod_{j=1}^{n} \theta\left(t^{n+1} t_{j}^{-1} \prod_{k=1}^{n+3} t_{k}\right)_{-\lambda_{j}}} \\
& = \begin{cases}\frac{\theta(1)_{-N}}{\theta\left(t^{n / 2}\right)_{-N} \prod_{n+1 \leqslant i<j \leqslant n+3} \theta\left(t^{(n+2) / 2} t_{i} t_{j}\right)_{-N}}, & n \text { четно, } \\
\frac{\theta(1)_{-N}}{\prod_{i=n+1}^{n+3} \theta\left(t^{(n+1) / 2} t_{i}\right)_{-N} \theta\left(t^{(n+3) / 2} \prod_{i=n+1}^{n+3} t_{i}\right)_{-N}}, & n \text { нечетно. }\end{cases}
\end{aligned}
$$

Предполагается, что эта формула связана с суммами вычетов для $A_{n}$-интеграла (10.13). При $p \rightarrow 0$ возникает формула суммирования для многократного ${ }_{8} \varphi_{7}$-ряда Густафсона-Ракха [100].

\section{2. Преобразования симметрии для многократных интегралов}

Общие эллиптические гипергеометрические интегралы типа I для системы корней $C_{n}$ имеют вид

$$
\begin{aligned}
& I_{n}^{(m)}\left(t_{1}, \ldots, t_{2 n+2 m+4}\right) \\
& \quad=\kappa_{n} \int_{\mathbb{T}^{n}} \prod_{1 \leqslant i<j \leqslant n} \frac{1}{\Gamma\left(z_{i}^{ \pm 1} z_{j}^{ \pm 1} ; p, q\right)} \prod_{j=1}^{n} \frac{\prod_{i=1}^{2 n+2 m+4} \Gamma\left(t_{i} z_{j}^{ \pm 1} ; p, q\right)}{\Gamma\left(z_{j}^{ \pm 2} ; p, q\right)} \frac{d z_{j}}{z_{j}},
\end{aligned}
$$

где $\left|t_{j}\right|<1$,

$$
\prod_{j=1}^{2 n+2 m+4} t_{j}=(p q)^{m+1}, \quad \kappa_{n}=\frac{(p ; p)_{\infty}^{n}(q ; q)_{\infty}^{n}}{(4 \pi i)^{n} n !}
$$

При $n=0$ мы полагаем $I_{0}^{(m)}=1$. Интеграл $I_{1}^{(1)}$ совпадает с $V$-функцией, a $I_{n}^{(0)}$ - с эллиптическим бета-интегралом (10.1). При вырождении $I_{n}^{(m)}$-функций до уровня обычного бета-интеграла они сводятся к интегралам Диксона [28], [105]. В [34] Рэйнс доказал следующую формулу преобразования:

$$
I_{n}^{(m)}\left(t_{1}, \ldots, t_{2 n+2 m+4}\right)=\prod_{1 \leqslant r<s \leqslant 2 n+2 m+4} \Gamma\left(t_{r} t_{s} ; p, q\right) I_{m}^{(n)}\left(\frac{\sqrt{p q}}{t_{1}}, \ldots, \frac{\sqrt{p q}}{t_{2 n+2 m+4}}\right) .
$$

Она является прямым обобщением третьего преобразования симметрии для $V$-функции, описанного в (5.4).

В [59] найдено следующее представление в виде определителя:

$$
\begin{aligned}
& I_{n}^{(m)}\left(t_{1}, \ldots, t_{2 n+2 m+4}\right)=\prod_{1 \leqslant i<j \leqslant n} \frac{1}{a_{j} \theta\left(a_{i} a_{j}^{ \pm 1} ; p\right) b_{j} \theta\left(b_{i} b_{j}^{ \pm 1} ; q\right)} \\
& \quad \times \operatorname{det}_{1 \leqslant i, j \leqslant n}\left(\kappa \int_{\mathbb{T}} \frac{\prod_{r=1}^{2 n+2 m+4} \Gamma\left(t_{r} z^{ \pm 1} ; p, q\right)}{\Gamma\left(z^{ \pm 2} ; p, q\right)} \prod_{k \neq i} \theta\left(a_{k} z^{ \pm 1} ; p\right) \prod_{k \neq j} \theta\left(b_{k} z^{ \pm 1} ; q\right) \frac{d z}{z}\right),
\end{aligned}
$$


где $a_{j}, b_{j}$ - произвольные параметры. При выборе $a_{i}=t_{j}, b_{j}=t_{n+j}, j=$ $1, \ldots, n$, возникает определитель матрицы

$$
T_{q}\left(t_{i}\right)^{-1} T_{p}\left(t_{n+j}\right)^{-1} I_{1}^{(m+n-1)}\left(q t_{1}, \ldots, q t_{n}, p t_{n+1}, \ldots, p t_{2 n}, t_{2 n+1}, \ldots, t_{2 n+2 m+4}\right),
$$

где $T_{q}\left(t_{k}\right)$ - оператор $q$-сдвига, $T_{q}\left(t_{k}\right) f\left(t_{k}\right)=f\left(q t_{k}\right)$. Таким образом, при $m=0$ мы получаем точно вычисляемый определитель однократных эллиптических гипергеометрических интегралов.

Для доказательства (12.2) необходимо записать

$$
\prod_{1 \leqslant i<j \leqslant n} \frac{1}{\Gamma\left(z_{i}^{ \pm 1} z_{j}^{ \pm 1} ; p, q\right)}=\prod_{1 \leqslant i<j \leqslant n} z_{i}^{-1} \theta\left(z_{i} z_{j}^{ \pm 1} ; p\right) \prod_{1 \leqslant i<j \leqslant n} z_{i}^{-1} \theta\left(z_{i} z_{j}^{ \pm 1} ; q\right)
$$

и связать оба множителя в правой части с эллиптическим аналогом определителя Коши (9.4). Использование формулы Гейне

$$
\frac{1}{n !} \int \operatorname{det}_{1 \leqslant i, j \leqslant n} \phi_{i}\left(z_{j}\right) \operatorname{det}_{1 \leqslant i, j \leqslant n} \psi_{i}\left(z_{j}\right) \prod_{1 \leqslant i \leqslant n} d \mu\left(z_{i}\right)=\operatorname{det}_{1 \leqslant i, j \leqslant n} \int \phi_{i}(z) \psi_{j}(z) d \mu(z)
$$

с $\phi_{i}\left(z_{j}\right)=a_{i} / \theta\left(a_{i} z_{j}^{ \pm 1} ; p\right)$ и $\psi_{i}\left(z_{j}\right)=b_{i} / \theta\left(b_{i} z_{j}^{ \pm 1} ; q\right)$ дает необходимый результат.

Имеет место $(n+2)$-членное рекуррентное соотношение [33], [59]

$$
\sum_{i=1}^{n+2} \frac{t_{i}}{\prod_{j=1, \neq i}^{n+2} \theta\left(t_{i} t_{j}^{ \pm 1} ; p\right)} T_{q}\left(t_{i}\right) I_{n}^{(m)}\left(t_{1}, \ldots, t_{2 n+2 m+4}\right)=0,
$$

где $\prod_{j=1}^{2 n+2 m+4} t_{j}=(p q)^{m} p$. Действительно, если мы запишем такое же рекуррентное соотношение для ядра интеграла $I_{n}^{(m)}$, то оно сведется к тождеству (9.3) после выделения общего множителя. Интегрируя последнее соотношение по подходящим контурам, мы получим (12.3).

Представление в виде определителя позволяет вывести другое $(m+2)$-членное рекуррентное соотношение

$$
\sum_{k=1}^{m+2} \frac{\prod_{m+3 \leqslant i \leqslant 2 n+2 m+4} \theta\left(t_{i} t_{k} / q ; p\right)}{t_{k} \prod_{1 \leqslant i \leqslant m+2 ; i \neq k} \theta\left(t_{i} / t_{k} ; p\right)} T_{q}\left(t_{k}\right)^{-1} I_{n}^{(m)}\left(t_{1}, \ldots, t_{2 n+2 m+4}\right)=0,
$$

где $t_{1} \cdots t_{2 m+2 n+4}=(p q)^{m+1} q$. Отметим, что преобразование $(12.1)$ переводит уравнение (12.3) в (12.4) и наоборот. Поэтому анализ совместных решений этих уравнений предоставляет альтернативный способ доказательства преобразования (12.1) [59].

Рассмотрим преобразования симметрии для других многомерных интегралов. Для девяти параметров $t, t_{1}, \ldots, t_{8} \in \mathbb{C}$, удовлетворяющих ограничениям $|t|,\left|t_{j}\right|<1$ и $t^{2 n-2} \prod_{j=1}^{8} t_{j}=p^{2} q^{2}$, определим интеграл

$$
\begin{aligned}
& I\left(t_{1}, \ldots, t_{8} ; t ; p, q\right)=\prod_{1 \leqslant j<k \leqslant 8} \Gamma\left(t_{j} t_{k} ; p, q, t\right) \\
& \quad \times \kappa_{n} \int_{\mathbb{T}^{n}} \prod_{1 \leqslant j<k \leqslant n} \frac{\Gamma\left(t z_{j}^{ \pm 1} z_{k}^{ \pm 1} ; p, q\right)}{\Gamma\left(z_{j}^{ \pm 1} z_{k}^{ \pm 1} ; p, q\right)} \prod_{j=1}^{n} \frac{\prod_{k=1}^{8} \Gamma\left(t_{k} z_{j}^{ \pm 1} ; p, q\right)}{\Gamma\left(z_{j}^{ \pm 2} ; p, q\right)} \frac{d z_{j}}{z_{j}} .
\end{aligned}
$$


Здесь

$$
\Gamma(z ; p, q, t)=\prod_{j, k, l=0}^{\infty}\left(1-z t^{j} p^{k} q^{l}\right)\left(1-z^{-1} t^{j+1} p^{k+1} q^{l+1}\right)
$$

есть эллиптическая гамма-функция более высокого уровня, связанная с гаммафункцией Барнса $\Gamma_{4}(u ; \boldsymbol{\omega})$ и удовлетворяющая уравнению

$$
\Gamma(t z ; p, q, t)=\Gamma(z ; p, q) \Gamma(z ; p, q, t)
$$

Функция (12.5) обобщает $V$-функцию на интегралы типа II для системы корней $B C_{n}$. В [34] Рэйнс доказал следующую формулу преобразования симметрии:

$$
I\left(t_{1}, \ldots, t_{8} ; t ; p, q\right)=I\left(s_{1}, \ldots, s_{8} ; t ; p, q\right)
$$

где

$$
\left\{\begin{array}{ll}
s_{j}=\rho^{-1} t_{j}, & j=1,2,3,4 \\
s_{j}=\rho t_{j}, & j=5,6,7,8
\end{array} ; \quad \rho=\sqrt{\frac{t_{1} t_{2} t_{3} t_{4}}{p q t^{1-n}}}=\sqrt{\frac{p q t^{1-n}}{t_{5} t_{6} t_{7} t_{8}}}, \quad|t|,\left|t_{j}\right|,\left|s_{j}\right|<1 .\right.
$$

Она описывает обобщение ключевого соотношения для $E_{7}$-группы (5.2), т. е. интеграл (12.5) инвариантен относительно всех преобразований этой группы. Как показано в [58], функция $I\left(t_{1}, \ldots, t_{8} ; t ; p, q\right)$ возникает в квантовой многочастичной модели [62] при определенном ограничении на параметры (условии балансировки) в качестве нормировки специальной собственной функции гамильтониана.

Определим общие эллиптические гипергеометрические интегралы типа I на системе корней $A_{n}$ :

$$
\begin{aligned}
I_{n}^{(m)}( & \left.s_{1}, \ldots, s_{n+m+2} ; t_{1}, \ldots, t_{n+m+2} ; A\right) \\
& =\kappa_{n}^{A} \int_{\mathbb{T}^{n}} \prod_{1 \leqslant j<k \leqslant n+1} \frac{1}{\Gamma\left(z_{j} z_{k}^{-1}, z_{j}^{-1} z_{k} ; p, q\right)} \prod_{j=1}^{n+1} \prod_{l=1}^{n+m+2} \Gamma\left(s_{l} z_{j}^{-1}, t_{l} z_{j} ; p, q\right) \frac{d z}{z},
\end{aligned}
$$

где $\left|t_{j}\right|,\left|s_{j}\right|<1$,

$$
\prod_{j=1}^{n+1} z_{j}=1, \quad \prod_{l=1}^{n+m+2} s_{l} t_{l}=(p q)^{m+1}
$$

и полагается $I_{0}^{(m)}=\prod_{l=1}^{m+2} \Gamma\left(s_{l}, t_{l} ; p, q\right)$. Используя результат точного вычисления $I_{n}^{(0)}$-интеграла $(10.11)$, можно вывести следующее рекурсивное соотношение для $I_{n}^{(m)}$-интегралов по индексу $m$ :

$$
\begin{aligned}
& I_{n}^{(m+1)}\left(s_{1}, \ldots, s_{n+m+3} ; t_{1}, \ldots, t_{n+m+3} ; A\right) \\
& \quad=\frac{\kappa_{n}^{A}}{\Gamma\left(v^{n+1} ; p, q\right)} \prod_{l=1}^{n+2} \frac{\Gamma\left(t_{n+m+3} s_{l} ; p, q\right)}{\Gamma\left(v^{-n-1} t_{n+m+3} s_{l} ; p, q\right)} \int_{\mathbb{T}^{n}} \prod_{1 \leqslant j<k \leqslant n+1} \frac{1}{\Gamma\left(w_{j} w_{k}^{-1}, w_{j}^{-1} w_{k} ; p, q\right)}
\end{aligned}
$$




$$
\begin{aligned}
& \times \prod_{j=1}^{n+1} \Gamma\left(v^{-n} t_{n+m+3} w_{j} ; p, q\right) \prod_{l=1}^{n+2} \Gamma\left(v^{-1} s_{l} w_{j}^{-1} ; p, q\right) \\
& \times I_{n}^{(m)}\left(v w_{1}, \ldots, v w_{n+1}, s_{n+3}, \ldots, s_{n+m+3} ; t_{1}, \ldots, t_{n+m+2} ; A\right) \frac{d w_{1}}{w_{1}} \ldots \frac{d w_{n}}{w_{n}},
\end{aligned}
$$

где

$$
v^{n+1}=\frac{t_{n+m+3}}{p q} \prod_{k=1}^{n+2} s_{k}=\frac{(p q)^{m+1}}{\prod_{k=1}^{n+m+2} t_{k} \prod_{l=n+3}^{n+m+3} s_{l}} .
$$

При $m=0 I_{n}^{(0)}$-интеграл в правой части вычисляется и дает преобразование симметрии [11]

$$
\begin{gathered}
I_{n}^{(1)}\left(s_{1}, \ldots, s_{n+3} ; t_{1}, \ldots, t_{n+3} ; A\right)=\prod_{k=1}^{n+2} \Gamma\left(t_{n+3} s_{k}, \frac{\prod_{i=1}^{n+2} s_{i}}{s_{k}}, s_{n+3} t_{k}, \frac{\prod_{i=1}^{n+2} t_{i}}{t_{k}} ; p, q\right) \\
\times I_{n}^{(1)}\left(v^{-1} s_{1}, \ldots, v^{-1} s_{n+2}, v^{n} s_{n+3} ; v t_{1}, \ldots, v t_{n+2}, v^{-n} t_{n+3} ; A\right),
\end{gathered}
$$

где $\prod_{k=1}^{n+3} t_{k} s_{k}=(p q)^{2}$ и

$$
v^{n+1}=\frac{t_{n+3}}{p q} \prod_{k=1}^{n+2} s_{k}=\frac{p q}{s_{n+3} \prod_{k=1}^{n+2} t_{k}} .
$$

Поскольку левая часть этого соотношения симметрична по параметрам $t_{k}$ или $s_{k}$, такая же $\left(S_{n+3} \times S_{n+3}\right)$-симметрия справедлива и для правой части, что приводит к дополнительным нетривиальным преобразованиям [34]. Удобно обозначить

$$
\begin{aligned}
I(v)= & \prod_{k=1}^{n+2} \Gamma\left(v^{-n-1} s_{n+3} s_{k}, v^{n+1} t_{n+3} t_{k} ; p, q\right) \\
& \quad \times I_{n}^{(1)}\left(v s_{1}, \ldots, v s_{n+2}, v^{-n} t_{n+3} ; v^{-1} t_{1}, \ldots, v^{-1} t_{n+2}, v^{n} s_{n+3} ; A\right),
\end{aligned}
$$

где все аргументы $I_{n}^{(1)}$ лежат внутри единичной окружности, $\prod_{k=1}^{n+3} t_{k}=$ $\prod_{k=1}^{n+3} s_{k}=p q$ и $v-$ произвольный свободный параметр (общее число свободных параметров равно $2 n+5)$. Тогда полученное соотношение может быть переписано в виде $I(v)=I\left(v^{-1}\right)$.

Другой тип преобразования для $I_{n}^{(m)}$-интегралов найден Рэйнсом [34]. Обозначим $T=\prod_{j=1}^{n+m+2} t_{j}, S=\prod_{j=1}^{n+m+2} s_{j}$, так что $S T=(p q)^{m+1}$, и пусть все $\left|t_{k}\right|,\left|s_{k}\right|,\left|T^{1 /(m+1)} / t_{k}\right|,\left|S^{1 /(m+1)} / s_{k}\right|<1$. Тогда справедливо следующее преобразование симметрии:

$$
\begin{aligned}
& I_{n}^{(m)}\left(t_{1}, \ldots, t_{n+m+2} ; s_{1}, \ldots, s_{n+m+2} ; A\right)=\prod_{j, k=1}^{n+m+2} \Gamma\left(t_{j} s_{k} ; p, q\right) \\
& \quad \times I_{m}^{(n)}\left(\frac{T^{1 /(m+1)}}{t_{1}}, \ldots, \frac{T^{1 /(m+1)}}{t_{n+m+2}} ; \frac{S^{1 /(m+1)}}{s_{1}}, \ldots, \frac{S^{1 /(m+1)}}{s_{n+m+2}} ; A\right) .
\end{aligned}
$$

Это соотношение обобщает преобразование (5.3), но естественные аналоги других $E_{7}$-отражений отсутствуют. Таким образом, симметрии $V$-функции, 
порожденные различными элементами группы Вейля для исключительной системы корней $E_{7}$, имеют многомерные аналоги. Однако различные отражения обобщаются на разные интегралы, ядра которых обладают симметриями по переменным интегрирования, связанными с различными корневыми системами.

\section{3. Заключение}

Несмотря на достаточно большой объем, многие проблемы рассмотрены в этом обзоре только фрагментарно, значительная часть утверждений приведена без доказательств, а ряд интересных вопросов вообще не затронут. Перечислим некоторые пропущенные достижения теории эллиптических гипергеометрических функций и укажем несколько открытых важных проблем.

Предположим, что существует конечно-разностный оператор первого порядка, переводящий одни рациональные функции в другие с меньшим числом полюсов ("понижающий" оператор). В работе [106] показано, что это возможно только при условии, что полюсы этих рациональных функций параметризуются общей эллиптической функцией второго порядка, а сама проблема связана с отображением Понселе.

Описание связи между биортогональными рациональными функциями и аппроксимацией Паде с предписанными нулями и полюсами [70], [106], [107] нами пропущено. Так, в работе [107] показано, что ${ }_{12} V_{11}$-эллиптический гипергеометрический ряд появляется в интерполяционных таблицах Паде для некоторых функций.

Естественно ожидать, что многократные эллиптические бета-интегралы определяют меру в соотношениях биортогональности для некоторых функций многих переменных, обобщающих одномерные соотношения (7.8). Первую систему таких многомерных функций, основанную на эллиптическом аналоге интеграла Сельберга (10.8), построил Рэйнс [34], [35] с помощью повышающих и понижающих операторов. В определенных пределах эти функции вырождаются в ортогональные многочлены Макдональда [98], Корнвиндера [108] или интерполирующие многочлены Окунькова [109] (о связи с последними упомянутыми многочленами см. также [102]). Результаты работ [34], [35] представляют собой наиболее передовые достижения теории эллиптических гипергеометрических функций многих переменных. Другой тип обобщения многочленов Макдональда на тэта-функциональный уровень предложен в [110].

Имеется красивая геометрическая интерпретация некоторых эллиптических гипергеометрических функций в терминах динамики на алгебраических поверхностях. В [111] Сакаи дал классификацию дискретных уравнений Пенлеве, связанных с аффинными группами Вейля. На вершине этой схемы лежит эллиптическое уравнение Пенлеве, связанное с системой корней $\hat{E}_{8}$. В работе [112] это уравнение детально изучено и найдена его редукция к эллиптическому гипергеометрическому уравнению. Соответственно было указано, что эллиптический гипергеометрический ряд ${ }_{12} V_{11}$ определяет частное решение эллиптического уравнения Пенлеве. Аналогичную роль играют общее решение 
эллиптического гипергеометрического уравнения [31], [58] и $B C_{n}$-эллиптический гипергеометрический интеграл типа II при специальных значениях параметров [91].

Подход Похгаммера и Хорна к функциям гипергеометрического типа [1], [2], которым мы воспользовались в случае функций одной переменной, еще не обобщен на эллиптические гипергеометрические функции многих переменных. Для этого необходимо научиться решать системы разностных уравнений первого порядка для ядер многократных рядов и интегралов с коэффициентами, являющимися эллиптическими функциями по всем переменным суммирования или интегрирования [10], [11]. Эта задача весьма сложна, и все примеры, рассмотренные выше, построены, исходя из других конструктивных соображений. На этом пути возникает общая задача классификации всех типов эллиптических бета-интегралов и их многопараметрических расширений на функции более высокого порядка. В частности, ожидается, что существуют эллиптические обобщения многократных $q$-бета-интегралов для исключительных систем корней [113]. Отметим также, что все рассмотренные выше многократные интегралы являются интегралами по полициклам при подходящих ограничениях на параметры. Интересно было бы рассмотреть интегралы по более сложным областям комплексных переменных интегрирования.

В работе [9] рассмотрена некоторая нелинейная дискретная интегрируемая система и предложена автомодельная редукция соответствующих уравнений, приводящая к эллиптическим решениям со многими параметрами. При этом была построена система дискретных биортогональных функций, выражающихся через ${ }_{12} V_{11}$-ряды. Биортогональные функции, описанные в п. 7.1, представляют собой только частный случай этих более общих функций, связанных с комбинациями нескольких ${ }_{12} V_{11}$-рядов и содержащих три дополнительных параметра. Подробный анализ свойств этих функций еще не проведен. Среди открытых проблем, связанных с общим решением эллиптического гипергеометрического уравнения, упомянем поиск явного вида необрывающейся эллиптической гипергеометрической цепной дроби и построение эллиптических аналогов ассоциированных многочленов Аски-Вильсона [114].

Различные обобщения интегрального преобразования (6.9) на многомерные интегралы на корневых системах предложены в [36], но часть соответствующих формул обращения еще не доказана. Проблема сходимости бесконечных эллиптических гипергеометрических рядов требует глубокого анализа. Необходимо понять, в каком смысле могут существовать такие функции. На данный момент совершенно не ясно, каковы эллиптические аналоги теоретико-числовых свойств обычных гипергеометрических функций. Необходимо также выяснить, можно ли построить нетривиальные функции гипергеометрического типа для римановых поверхностей более высокого рода (простейший пример функций такого типа приведен в [115]).

В заключение можно констатировать, что основные структурные элементы теории обычных и $q$-гипергеометрических функций имеют свои естественные эллиптические аналоги. Более того, различные "старые" гипергеометрические понятия приобретают новый смысл, связанный со свойствами эллиптических функций. В настоящее время известно достаточно большое число примене- 
ний эллиптических гипергеометрических функций в математической физике: в точно решаемых моделях статистической механики, связанных с эллиптическими решениями уравнения Янга-Бакстера [8] и алгеброй Склянина [35], [79], [80], [116], в интегрируемых нелинейных цепочках с дискретным временем [9], в релятивистских квантовых многочастичных моделях типа Калоджеро-Сазерленда [58] и в нелинейных дискретных уравнениях типа Пенлеве [91], [112]. Естественно ожидать, что со временем количество таких приложений будет расти и, кроме того, найдутся новые концептуальные пересечения с другими разделами математики.

Я глубоко признателен своим соавторам О. Варнаару, Я.Ф. ван Диежену, А. С. Жеданову и Э. М. Рэйнсу за плодотворное сотрудничество и многочисленные стимулирующие дискуссии. В процессе работы над теорией эллиптических гипергеометрических функций были полезными обсуждения различных проблем с Х. Розенгреном, С. Руджинарсом и М. Шлоссером, внесшими свой существенный вклад в развитие этой теории, а также с Р. Аски, В. В. Зудилиным, К. Кратенталером, А. Левиным, Ю. И. Маниным, В. Б. Приезжевым, Д. Цагиром и Дж. Е. Эндрюсом. Этот обзор частично основан на докторской диссертации автора [31] и лекционных записках вводного курса, прочитанного в Независимом московском университете осенью 2005 г. Некоторые представленные результаты были получены во время визитов в Институт математики имени Макса Планка (Бонн), руководству которого я признателен за гостеприимство. Данная работа частично поддержана Российским фондом фундаментальных исследований, грант № 08-01-00392.

\section{Приложение А. Эллиптические функции и тэта-функции Якоби}

Периоды некоторой периодической функции называются примитивными, если их линейные комбинации с целыми коэффициентами дают все периоды этой функции. Хорошо известно, что нетривиальные мероморфные периодические функции не могут иметь больше двух примитивных периодов [117]. Функции действительной переменной могут иметь только один примитивный период. Эти утверждения использовались при выводе выражения (2.21) и построении эллиптических аналогов функции Мейера. Мероморфные функции $f(u)$ с двумя примитивными периодами называются эллиптическими функциями, т. е. существуют $\omega_{1}, \omega_{2} \in \mathbb{C}, \operatorname{Im}\left(\omega_{1} / \omega_{2}\right) \neq 0$, и $f\left(u+\omega_{1}\right)=f\left(u+\omega_{2}\right)=f(u)$.

Примитивные периоды эллиптической функции $f(u)$ образуют параллелограммы периодов и $f(u)$ определяется своими значениями внутри них и на паре смежных ребер. Назовем фундаментальной областью $D$ внутренность одного из этих параллелограммов, выбранного так, что на его границе $\partial D$ нет точек дивизора $f(u)$. Очевидно, что $f(u)$ (как мероморфная функция) имеет конечное число нулей и полюсов в $D$.

Интеграл $(2 \pi i)^{-1} \int_{\partial D} \frac{f^{\prime}(u)}{f(u)} d u$ определяет число нулей в области $D$ для целых функций и разность числа нулей и полюсов для мероморфных функций. Из-за периодичности этот интеграл равен нулю для эллиптических функций, т. е. для них число нулей в $D$ равно числу полюсов. Это число нулей $s$ (или полюсов) называется порядком эллиптической функции. Равенство 
$f(u)=C$, где $C$ - произвольная наперед заданная постоянная, выполняется в $D$ ровно $s$ раз (т. е. $f(u)$ есть $s$-листная функция). Это следует из того, что у эллиптической функции $f(u)-C$ имеется $s$ полюсов в $D$ и ровно $s$ нулей соответственно.

Сумма вычетов полюсов $f(u)$ в $D$ равна нулю, что следует из равенства $\int_{\partial D} f(u) d u=0$. Поэтому не существует эллиптических функций порядка $s=1$, а эллиптическая функция нулевого порядка есть постоянная (теорема Лиувилля). Это дает способ доказательства тождеств для эллиптических функций - если разность функций $f_{1}(u)-f_{2}(u)$ (или отношение $f_{1}(u) / f_{2}(u)$ ) эллиптична и содержит не более одного полюса в фундаментальной области, то $f_{1}(u)-f_{2}(u)=$ const (или $f_{1}(u) / f_{2}(u)=$ const).

Хорошо известная эллиптическая функция Вейерштрасса $\wp\left(u \mid \omega_{1}, \omega_{2}\right)$ [117] имеет в фундаментальной области один полюс второго порядка, т. е. $s=2$. Пара $(x, y)=\left(\wp(u), \wp^{\prime}(u)\right)$ определяет униформизацию эллиптической кривой $y^{2}=4 x^{3}-g_{2} x-g_{3}$. Эллиптические функции образуют дифференциальное поле, и любые две эллиптические функции $f(u)$ и $g(u)$ связаны алгебраическим соотношением $P(f, g)=0$, где $P(f, g)$ - некоторый многочлен своих аргументов. При выборе $g(u)=f^{\prime}(u)$ видно, что любая эллиптическая функция удовлетворяет нелинейному дифференциальному уравнению первого порядка $P\left(f, f^{\prime}\right)=0$. Взяв $g(u)=f(u+y)$, получаем $P(f, g)=\sum_{k=0}^{N} p_{k}(f(u), y) \times$ $f(u+y)^{k}=0$, где $p_{k}(f(u), y)$ - некоторые многочлены по $f(u)$ с коэффициентами, зависящими от $y$. Переставив $u$ и $y$, получаем, что $p_{k}(f(y), u)=p_{k}(f(u), y)$ суть симметричные многочлены $f(u)$ и $f(y)$ с постоянными коэффициентами. Поэтому условие $P(f, g)=0$ переписывается в виде $Q(f(u), f(y), f(u+y))=0$, где $Q$ - некоторый многочлен трех аргументов. При выполнении такого соотношения говорят, что функция $f(u)$ обладает алгебраической теоремой сложения. Вейерштрасс показал, что мероморфная функция $f(u)$, обладающая такой теоремой сложения, является либо эллиптической функцией, либо ее вырождением к тригонометрическим или рациональным функциям.

Для более явного представления эллиптических функций необходимы тэтафункции. Произвольные целые функции $f(u)$ называются (эллиптическими) тэта-функциями, если

$$
f\left(u+\omega_{1}\right)=e^{a u+b} f(u), \quad f\left(u+\omega_{2}\right)=e^{c u+d} f(u)
$$

для некоторых $a, b, c, d \in \mathbb{C}$ и $\operatorname{Im}\left(\omega_{1} / \omega_{2}\right) \neq 0$. Заменой $u$ на $\omega_{2} u$ и умножением $f(u)$ на $e^{\alpha u^{2}+\beta u}$ с подобранными постоянными $\alpha$ и $\beta$ можно добиться равенств

$$
f(u+1)=f(u), \quad f(u+\tau)=e^{a u+b} f(u) .
$$

В этом приложении мы используем параметризацию $\tau=\omega_{1} / \omega_{2}$ и обозначаем $q=e^{2 \pi i \tau}$ (в отличие от основного текста обзора, где $\tau=\omega_{3} / \omega_{2}$ и $p=e^{2 \pi i \tau}$ ). При $a \neq 0$ параметр $b$ можно устранить сдвигом $u \rightarrow u-b / a$. Для параллелограмма $D$ с вершинами $(0,1,1+\tau, \tau)$ находим $\int_{\partial D} \frac{f^{\prime}(u)}{f(u)} d u=-a$. Поэтому $a=-2 \pi i s$, где величина $s=0,1,2, \ldots$ определяет число нулей $f(u)$ в $D$. Ключевая характеристика тэта-функции $s$ называется ее порядком. 
Периодичность $f(u+1)=f(u)$ позволяет разложить $f(u)$ в ряд Фурье $f(u)=$ $\sum_{j=-\infty}^{\infty} c_{j} e^{2 \pi i j u}$. Подставляя его во второе уравнение с $a=-2 \pi i s$ и решая возникающее рекуррентное соотношение на коэффициенты $c_{j}$, находим

$$
f(u)=\sum_{l=0}^{s-1} c_{l} z^{l} \sum_{k \in \mathbb{Z}} q^{s k(k-1) / 2}\left(q^{l} z^{s}\right)^{k}, \quad z=e^{2 \pi i u} .
$$

Коэффициенты $c_{0}, \ldots, c_{s-1}$ произвольны, т. е. тэта-функции порядка $s$ образуют $s$-мерное векторное пространство.

Если восстановить произвольные множители квазипериодичности, то тэтафункция без нулей равна $e^{P_{2}(u)}$, где $P_{2}(u)$ - некоторый многочлен второй степени. Тэта-функции первого порядка с одним нулем в фундаментальном параллелограмме квазипериодов называются тэта-функциями Якоби, а функции с $s>1$ называются тэта-функциями высшего уровня. При $s=1$ удобно работать с четырьмя тэта-функциями с характеристиками

$$
\theta_{a b}(u)=\sum_{k \in \mathbb{Z}} e^{\pi i \tau(k+a / 2)^{2}} e^{2 \pi i(k+a / 2)(u+b / 2)},
$$

в которых переменные $a$ и $b$ принимают значения 0 или 1. Стандартные тэтафункции Якоби определяются так [12]:

$$
\begin{aligned}
& \theta_{1}(u \mid \tau)=\theta_{1}(u)=-\theta_{11}(u), \\
& \theta_{2}(u \mid \tau)=\theta_{2}(u)=\theta_{10}(u)=\theta_{1}\left(u+\frac{1}{2}\right), \\
& \theta_{3}(u \mid \tau)=\theta_{3}(u)=\theta_{00}(u)=e^{\pi i \tau / 4+\pi i u} \theta_{1}\left(u+\frac{1}{2}+\frac{\tau}{2}\right), \\
& \theta_{4}(u \mid \tau)=\theta_{4}(u)=\theta_{01}(u)=-i e^{\pi i \tau / 4+\pi i u} \theta_{1}\left(u+\frac{\tau}{2}\right) .
\end{aligned}
$$

Отметим, что все они имеют вид $\sum_{k \in \mathbb{Z}} c_{k}$ с $h(k)=c_{k+1} / c_{k}=q^{k} y$ для некоторой постоянной $y$. Поскольку $h(k)$ рациональна по $q^{k}$, тэта-функции Якоби представляют собой специальный класс $q$-гипергеометрических функций.

$\theta_{1}(u)$-функция нечетна, $\theta_{1}(-u)=-\theta_{1}(u)$, и подчиняется условиям квазипериодичности

$$
\theta_{1}(u+1)=-\theta_{1}(u), \quad \theta_{1}(u+\tau)=-e^{-\pi i \tau-2 \pi i u} \theta_{1}(u) .
$$

Она связана с укороченной тэта-функцией $\theta(z ; q)=(z ; q)_{\infty}\left(q z^{-1} ; q\right)_{\infty}$ тождеством тройного произведения Якоби

$$
\theta_{1}(u)=i q^{1 / 8} e^{-\pi i u}(q ; q)_{\infty} \theta\left(e^{2 \pi i u} ; q\right)
$$

Трансформационные свойства $\theta_{1}$-функции относительно $P S L(2, \mathbb{Z})$-группы модулярных преобразований $\tau \rightarrow(a \tau+b) /(c \tau+d), a, b, c, d \in \mathbb{Z}, a d-b c=1$, определяются соотношениями [118]

$$
\theta_{1}(u \mid \tau+1)=e^{\pi i / 4} \theta_{1}(u \mid \tau), \quad \theta_{1}\left(\frac{u}{\tau} \mid \frac{-1}{\tau}\right)=-i \sqrt{-i \tau} e^{\pi i u^{2} / \tau} \theta_{1}(u \mid \tau) .
$$


Удобно использовать обозначения

$$
\theta_{a}\left(u_{1}, \ldots, u_{k}\right):=\theta_{a}\left(u_{1}\right) \cdots \theta_{a}\left(u_{k}\right) \quad \text { и } \quad \theta_{a}(x \pm y):=\theta_{a}(x+y, x-y) .
$$

Тогда формула удвоения аргумента имеет вид

$$
\theta_{1}(2 u)=\frac{i q^{1 / 8}}{(q ; q)_{\infty}^{3}} \theta_{1}\left(u, u+\frac{1}{2}, u+\frac{\tau}{2}, u-\frac{1+\tau}{2}\right) .
$$

Теорема сложения для тэта-функций, называемая иногда соотношением Римана, использует произведения четырех тэта-функций

$$
\theta_{1}(u \pm a, v \pm b)-\theta_{1}(u \pm b, v \pm a)=\theta_{1}(a \pm b, u \pm v)
$$

или

$$
\theta\left(x w^{ \pm 1}, y z^{ \pm 1} ; p\right)-\theta\left(x z^{ \pm 1}, y w^{ \pm 1} ; p\right)=y w^{-1} \theta\left(x y^{ \pm 1}, w z^{ \pm 1} ; p\right) .
$$

Доказательство этого равенства элементарно. Отношение выражений, стоящих в его левой и правой частях, есть ограниченная функция $x \in \mathbb{C}^{*}$ (оно инвариантно относительно замены $x \rightarrow p x$ и не содержит полюсов в кольце $|p| \leqslant|x| \leqslant 1$ ). По теореме Лиувилля это отношение не зависит от $x$, а при $x=w$ оно равно 1 .

Любая тэта-функция $s$-го порядка $f(u)$ с квазипериодами $\omega_{1}, \omega_{2}$ и координатами нулей в фундаментальной области $a_{1}, \ldots, a_{s}$ может быть представлена в виде

$$
f(u)=e^{P_{2}(u)} \prod_{k=1}^{s} \theta_{1}\left(\frac{u-a_{k}}{\omega_{2}} \mid \frac{\omega_{1}}{\omega_{2}}\right),
$$

где $P_{2}(u)$ - некоторый многочлен $u$ второго порядка. На самом деле, функция

$$
g(u)=\prod_{k=1}^{s} \theta_{1}\left(\frac{u-a_{k}}{\omega_{2}} \mid \frac{\omega_{1}}{\omega_{2}}\right)
$$

является тэта-функцией $s$-го порядка с теми же нулями в параллелограмме квазипериодов $\omega_{1}$ и $\omega_{2}$, что и функция $f(u)$. Поэтому отношение $f(u) / g(u)$ является целой функцией без нулей и полюсов, точнее, тэта-функцией нулевого порядка, т. е. $e^{P_{2}(u)}$. В [118] предложена последовательная теория форм Якоби - функций, обладающих трансформационными свойствами, схожими со свойствами функций (А.6). Отметим, что все векторы $f_{j}(u), j=1, \ldots, s$, любого базиса пространства тэта-функций $s$-го порядка представимы в указанном виде с матрицей нулей $a_{j k}$, удовлетворяющей ряду ограничений (например, $\sum_{k=1}^{s} a_{j k}=$ const).

Назовем мероморфными тэта-функциями отношения тэта-функций произвольного конечного порядка. Легко видеть, что они определяют мероморфные решения уравнений (А.1). Для этого обозначим через $a_{1}, \ldots, a_{n}$ координаты нулей, а через $b_{1}, \ldots, b_{m}$ координаты полюсов соответствующей функции $f(u)$ в фундаментальной области. Тогда отношение

$$
\frac{f(u) \prod_{k=1}^{m} \theta_{1}\left(\frac{u-b_{k}}{\omega_{2}} \mid \frac{\omega_{1}}{\omega_{2}}\right)}{\prod_{k=1}^{n} \theta_{1}\left(\frac{u-a_{k}}{\omega_{2}} \mid \frac{\omega_{1}}{\omega_{2}}\right)}
$$


является целой функцией без нулей, удовлетворяющей уравнениям $f\left(u+\omega_{1}\right)=$ $e^{a^{\prime} u+b^{\prime}} f(u)$ и $f\left(u+\omega_{2}\right)=e^{c^{\prime} u+d^{\prime}}$ с некоторыми $a^{\prime}, b^{\prime}, c^{\prime}, d^{\prime}$, т. е. тэта-функцией нулевого порядка $e^{P_{2}(u)}$.

Любая эллиптическая функция $f(u)$ конечного порядка $s$ с периодами $\omega_{1}, \omega_{2}$ может быть представлена в виде

$$
f(u)=C \prod_{k=1}^{s} \frac{\theta_{1}\left(\frac{u-a_{k}}{\omega_{2}} \mid \frac{\omega_{1}}{\omega_{2}}\right)}{\theta_{1}\left(\frac{u-b_{k}}{\omega_{2}} \mid \frac{\omega_{1}}{\omega_{2}}\right)},
$$

где $C$ - некоторая постоянная, а $a_{k}$ и $b_{k}$ обозначают координаты некоторых нулей и полюсов $f(u)$, конгруэнтных нулям и полюсам в фундаментальном параллелограмме периодов. При этом должно выполняться следующее ограничение на $a_{k}$ и $b_{k}$ :

$$
a_{1}+\cdots+a_{s}=b_{1}+\cdots+b_{s} \bmod \omega_{2} .
$$

Это утверждение следует из того, что обе части равенства (А.7) мероморфны и дважды периодичны. Поэтому их отношение будет ограниченной целой функцией, т. е. постоянной. Линейное ограничение на значения $a_{k}$ и $b_{k}(\mathrm{~A} .8)$, которое мы называем условием балансировки, возникает из условия сокращения множителей квазипериодичности $\theta_{1}$-функций, порожденных сдвигом $u \rightarrow u+\omega_{1}$.

Подставляя выражение (А.2) в (А.7) и обозначая $z=e^{2 \pi i u / \omega_{2}}, t_{k}=e^{-2 \pi i a_{k} / \omega_{2}}$, $w_{k}=e^{-2 \pi i b_{k} / \omega_{2}}$, получаем

$$
f(u)= \pm C \prod_{k=1}^{s} \frac{\theta\left(t_{k} z ; q\right)}{\theta\left(w_{k} z ; q\right)}, \quad \prod_{k=1}^{s} t_{k}=\prod_{k=1}^{s} w_{k},
$$

где неоднозначность в выборе знака возникла из-за множителя $e^{-\pi i u}$ в (А.2).

\section{Список литературы}

[1] G. E. Andrews, R. Askey, R. Roy, Special functions, Encyclopedia Math. Appl., 71, Cambridge Univ. Press, Cambridge, 1999.

[2] И. М. Гельфанд, М. И. Граев, В. С. Ретах, "Общие гипергеометрические системы уравнений и ряды гипергеометрического типа", УМH, 47:4 (1992), 3-82; англ. пер.: I. M. Gel'fand, M. I. Graev, V.S. Retakh, "General hypergeometric systems of equations and series of hypergeometric type", Russian Math. Surveys, 47:4 (1992), $1-88$.

[3] G. Gasper, M. Rahman, Basic hypergeometric series, 2nd ed., Encyclopedia Math. Appl., 96, Cambridge Univ. Press, Cambridge, 2004.

[4] Е.К. Склянин, Л. А. Тахтаджян, Л.Д. Фаддеев, "Квантовый метод обратной задачи. I", ТМФ, 40:2 (1979), 194-220; англ. пер.: E. K. Sklyanin, L. A. Takhtadzhyan, L. D. Faddeev, "Quantum inverse problem method. I", Theoret. Math. Phys., 40:2 (1979), 688-706.

[5] Л.А. Тахтаджян, Л.Д. Фаддеев, "Квантовый метод обратной задачи и $X Y Z$ модель Гейзенберга", УМH, 34:5 (1979), 13-63; англ. пер.: L. A. Takhtadzhan, L. D. Faddeev, "The quantum method of the inverse problem and the Heisenberg XYZ model", Russian Math. Surveys, 34:5 (1979), 11-68. 
[6] R. J. Baxter, "Partition function of the eight-vertex lattice model", Ann. Physics, 70:1 (1972), 193-228.

[7] E. Date, M. Jimbo, A. Kuniba, T. Miwa, M. Okado, "Exactly solvable SOS models, II. Proof of the star-triangle relation and combinatorial identities", Conformal field theory and solvable lattice models (Kyoto, 1986), Adv. Stud. Pure Math., 16, Academic Press, Boston, MA, 1988, 17-122.

[8] I. B. Frenkel, V.G. Turaev, "Elliptic solutions of the Yang-Baxter equation and modular hypergeometric functions", The Arnold-Gelfand mathematical seminars, Birkhäuser, Boston, MA, 1997, 171-204.

[9] V.P. Spiridonov, A.S. Zhedanov, "Spectral transformation chains and some new biorthogonal rational functions", Comm. Math. Phys., 210:1 (2000), 49-83.

[10] V.P. Spiridonov, "Theta hypergeometric series", Asymptotic combinatorics with application to mathematical physics (St. Petersburg, 2001), NATO Sci. Ser. II Math. Phys. Chem., 77, Kluwer, Dordrecht, 2002, 307-327; arXiv: math/0303204.

[11] V.P. Spiridonov, "Theta hypergeometric integrals", Алгебра и анализ, 15:6 (2003), 161-215; англ. изд.: V.P. Spiridonov, "Theta hypergeometric integrals", St. Petersburg Math. J., 15:6 (2004), 929-967; arXiv: math/0303205.

[12] Г. Бейтмен, А. Эрдейи, Высшие транси,ндентные функиии. Гипергеометрическая функиия. Ч. I: Функиии Лежандра, Наука, М., 1965; пер. с англ.: A. Erdélyi, W. Magnus, F. Oberhettinger, F. G. Tricomi, Higher transcendental functions, vol. I, McGraw-Hill, New York-Toronto-London, 1953.

[13] E. W. Barnes, "On the theory of the multiple gamma function", Cambr. Trans., 19 (1904), 374-425.

[14] F. H. Jackson, "The basic gamma-function and the elliptic functions", Proc. Roy. Soc. Lond. Ser. A, 76:508 (1905), 127-144.

[15] T. Shintani, "On a Kronecker limit formula for real quadratic field", J. Fac. Sci. Univ. Tokyo Sect. IA Math., 24:1 (1977), 167-199.

[16] N. Kurokawa, "Multiple sine functions and Selberg zeta functions", Proc. Japan Acad. Ser. A Math. Sci., 67:3 (1991), 61-64.

[17] L. D. Faddeev, "Discrete Heisenberg-Weyl group and modular group", Lett. Math. Phys., 34:3 (1995), 249-254.

[18] S. N. M. Ruijsenaars, "First order analytic difference equations and integrable quantum systems", J. Math. Phys., 38:2 (1997), 1069-1146.

[19] M. Jimbo, T. Miwa, "Quantum KZ equation with $|q|=1$ and correlation functions of the $X X Z$ model in the gapless regime", J. Phys. A, 29:12 (1996), 2923-2958.

[20] V. Tarasov, A. Varchenko, "Geometry of $q$-hypergeometric functions, quantum affine algebras and elliptic quantum groups", Astérisque, 1997, № 246, 1-135; arXiv: q-alg/9703044.

[21] L. D. Faddeev, R. M. Kashaev, A. Yu. Volkov, "Strongly coupled quantum discrete Liouville theory. I: Algebraic approach and duality", Commun. Math. Phys., 219:1 (2001), 199-219.

[22] B. Ponsot, J. Teschner, "Clebsch-Gordan and Racah-Wigner coefficients for a continuous series of representations of $U_{q}(s l(2, \mathbb{R}))$ ", Comm. Math. Phys., 224:3 (2001), 613-655.

[23] S. Kharchev, D. Lebedev, M. Semenov-Tian-Shansky, "Unitary representations of $U_{q}(s l(2, \mathbb{R}))$, the modular double and the multiparticle $q$-deformed Toda chains", Comm. Math. Phys., 225:3 (2002), 573-609.

[24] A. Yu. Volkov, "Noncommutative hypergeometry", Comm. Math. Phys., 258:2 (2005), 257-273.

[25] G. Felder, A. Varchenko, "The elliptic gamma function and $S L(3, \mathbb{Z}) \ltimes \mathbb{Z}^{3}$ ", $A d v$. Math., 156:1 (2000), 44-76. 
[26] E. Friedman, S. Ruijsenaars, "Shintani-Barnes zeta and gamma functions", Adv. Math., 187:2 (2004), 362-395.

[27] A. Narukawa, "The modular properties and the integral representations of the multiple elliptic gamma functions", Adv. Math., 189:2 (2004), 247-267.

[28] E. M. Rains, "Limits of elliptic hypergeometric integrals", Ramanujan J., to appear; arXiv: math/0607093, 2006.

[29] В.П. Спиридонов, “Об эллиптической бета-функции”, УМH, 56:1 (2001), 181-182; англ. пер.: V.P. Spiridonov, "On the elliptic beta function", Russian Math. Surveys, 56:1 (2001), 185-186.

[30] В. П. Спиридонов, “Дерево Бэйли для интегралов", ТМФ, 139:1 (2004), 104-111; англ. пер.: V.P. Spiridonov, "A Bailey tree for integrals", Theoret. and Math. Phys., 139:1 (2004), 536-541.

[31] В.П. Спиридонов, Эллиптические гипергеометрические функиии, Дис. ... докт. физ.-матем. наук, ЛТФ ОИЯИ, Дубна, 2004.

[32] J.F. van Diejen, V.P. Spiridonov, "An elliptic Macdonald-Morris conjecture and multiple modular hypergeometric sums", Math. Res. Lett., 7:5-6 (2000), 729-746.

[33] J. F. van Diejen, V. P. Spiridonov, "Elliptic Selberg integrals", Internat. Math. Res. Notices, 2001, № 20, 1083-1110.

[34] E. M. Rains, "Transformations of elliptic hypergeometric integrals", Ann. of Math. (to appear); arXiv: math/0309252.

[35] E. M. Rains, " $B C_{n}$-symmetric Abelian functions", Duke Math. J., 135:1 (2006), 99-180.

[36] V. P. Spiridonov, S. O. Warnaar, "Inversions of integral operators and elliptic beta integrals on root systems", Adv. Math., 207:1 (2006), 91-132.

[37] S. N. M. Ruijsenaars, "On Barnes' multiple zeta and gamma functions", Adv. Math., 156:1 (2000), 107-132.

[38] J. F. van Diejen, V.P. Spiridonov, "Unit circle elliptic beta integrals", Ramanujan J., 10:2 (2005), 187-204.

[39] M. Rahman, "An integral representation of a ${ }_{10} \phi_{9}$ and continuous bi-orthogonal ${ }_{10} \phi_{9}$ rational functions", Canad. J. Math., 38:3 (1986), 605-618.

[40] B. Nassrallah, M. Rahman, "Projection formulas, a reproducing kernel and a generating function for q-Wilson polynomials", SIAM J. Math. Anal., 16:1 (1985), 186-197.

[41] R. Askey, J. Wilson, "Some basic hypergeometric orthogonal polynomials that generalize Jacobi polynomials", Mem. Amer. Math. Soc., 54:319 (1985).

[42] R. Askey, "Beta integrals in Ramanujan's papers, his unpublished work and further examples", Ramanujan revisited (Urbana-Champaign, IL, 1987), Academic Press, Boston, MA, 1988, 561-590.

[43] V.P. Spiridonov, "Short proofs of the elliptic beta integrals", Ramanujan J., 13:1-3 (2007), 265-283; arXiv: math/0408369.

[44] H. S. Wilf, D. Zeilberger, "An algorithmic proof theory for hypergeometric (ordinary and "q") multisum/integral identities", Invent. Math., 108:3 (1992), 575-633.

[45] H. Rosengren, "A proof of a multivariable elliptic summation formula conjectured by Warnaar", q-series with applications to combinatorics, number theory, and physics (Urbana, IL, 2000), Contemp. Math., 291, Amer. Math. Soc., Providence, RI, 2001, 193-202; arXiv: math/0101073.

[46] V. P. Spiridonov, A. S. Zhedanov, "To the theory of biorthogonal rational functions", Sūrikaisekikenkyūsho Kōkyūroku, 2003, № 1302, 172-192.

[47] M. Schlosser, "Elliptic enumeration of nonintersecting lattice paths", J. Combin. Theory Ser. A, 114:3 (2007), 505-521. 
[48] M. Schlosser, A Taylor expansion theorem for an elliptic extension of the AskeyWilson operator, arXiv: 0803.2329.

[49] W. Chu, C. Jia, "Abel's method on summation by parts and theta hypergeometric series", J. Combin. Theory Ser. A (to appear).

[50] J. V. Stokman, "Hyperbolic beta integrals", Adv. Math., 190:1 (2005), 119-160.

[51] L. J. Slater, Generalized hypergeometric functions, Cambridge Univ. Press, Cambridge, 1966.

[52] V.P. Spiridonov, "An elliptic incarnation of the Bailey chain", Int. Math. Res. Not., 2002, № 37, 1945-1977.

[53] S. O. Warnaar, "Summation and transformation formulas for elliptic hypergeometric series", Constr. Approx., 18:4 (2002), 479-502.

[54] S. O. Warnaar, "Summation formulae for elliptic hypergeometric series", Proc. Amer. Math. Soc., 133:2 (2005), 519-527.

[55] G. Gasper, M. Schlosser, "Summation, transformation, and expansion formulas for multibasic theta hypergeometric series", Adv. Stud. Contemp. Math. (Kyungshang), 11:1 (2005), 67-84; arXiv: math/0505215.

[56] H. Rosengren, M. Schlosser, "On Warnaar's elliptic matrix inversion and Karlsson-Minton-type elliptic hypergeometric series", J. Comput. Appl. Math., 178:1-2 (2005), 377-391.

[57] A. Zhedanov, Elliptic polynomials orthogonal on the unit circle with a dense point spectrum, arXiv: 0711.4696.

[58] В.П. Спиридонов, "Эллиптические гипергеометрические функции и модели типа Калоджеро-Сазерленда", ТМФ, 150:2 (2007), 311-324; англ. пер: V.P. Spiridonov, "Elliptic hypergeometric functions and Calogero-Sutherland-type models", Theoret. Math. Phys., 150:2 (2007), 266-277.

[59] E. M. Rains, V.P. Spiridonov, Determinants of elliptic hypergeometric integrals, arXiv: 0712.4253 .

[60] S. N. M. Ruijsenaars, "Generalized hypergeometric function satisfying four analytic difference equations of Askey-Wilson type", Comm. Math. Phys., 206:3 (1999), 639-690.

[61] F. J. van de Bult, E. M. Rains, J. V. Stokman, "Properties of generalized univariate hypergeometric functions", Comm. Math. Phys., 275:1 (2007), 37-95; arXiv: math/0607250.

[62] J. F. van Diejen, "Integrability of difference Calogero-Moser systems", J. Math. Phys., 35:6 (1994), 2983-3004.

[63] Y. Komori, K. Hikami, "Quantum integrability of the generalized elliptic Ruijsenaars models", J. Phys. A, 30:12 (1997), 4341-4364.

[64] S. N. M. Ruijsenaars, "Complete integrability of relativistic Calogero-Moser systems and elliptic function identities", Comm. Math. Phys., 110:2 (1987), 191-213.

[65] V. I. Inozemtsev, "Lax representation with spectral parameter on a torus for integrable particle systems", Lett. Math. Phys., 17:1 (1989), 11-17.

[66] G. E. Andrews, "Bailey's transform, lemma, chains and tree", Special functions 2000: current perspective and future directions (Tempe, AZ), NATO Sci. Ser. II Math. Phys. Chem., 30, Kluwer, Dordrecht, 2001, 1-22.

[67] S. O. Warnaar, "Extensions of the well-poised and elliptic well-poised Bailey lemma", Indag. Math. (N.S.), 14:3-4 (2003), 571-588.

[68] D. M. Bressoud, "A matrix inverse", Proc. Amer. Math. Soc., 88:3 (1983), 446-448.

[69] X. R. Ma, "An extension of Warnaar's matrix inversion", Proc. Amer. Math. Soc., 133:11 (2005), 3179-3189. 
[70] A. Zhedanov, "Biorthogonal rational functions and the generalized eigenvalue problem", J. Approx. Theory, 101:2 (1999), 303-329.

[71] M. E. H. Ismail, D. R. Masson, "Generalized orthogonality and continued fractions", J. Approx. Theory, 83:1 (1995), 1-40.

[72] А. А. Гончар, "О скорости рациональной аппроксимации некоторых аналитических функций”, Матем. сб., 105:2 (1978), 147-163; англ. пер.: А. А. Gonchar, "On the speed of rational approximation of some analytic functions", Math. USSR-Sb., 34:2 (1978), 131-145.

[73] А.А. Гончар, Г. Лопес Лагомасино, "О теореме Маркова для многоточечных аппроксимаций Паде", Матем. сб., 105:4 (1978), 512-525; англ. пер.: A. A. Gonchar, G. López Lagomasino, "On Markov's theorem for multipoint Padé approximants", Math. USSR-Sb., 34:4 (1978), 449-459.

[74] V.P. Spiridonov, A.S. Zhedanov, "Classical biorthogonal rational functions on elliptic grids", C. R. Math. Acad. Sci. Soc. R. Can., 22:2 (2000), 70-76.

[75] J.A. Wilson, "Orthogonal functions from Gram determinants", SIAM J. Math. Anal., 22:4 (1991), 1147-1155.

[76] H. Rosengren, "An elementary approach to $6 j$-symbols (classical, quantum, rational, trigonometric, and elliptic)", Ramanujan J., 13:1-3 (2007), 131-166.

[77] D. P. Gupta, D. R. Masson, "Contiguous relations, continued fractions and orthogonality", Trans. Amer. Math. Soc., 350:2 (1998), 769-808.

[78] D. P. Gupta, D. R. Masson, "Watson's basic analogue of Ramanujan's entry 40 and its generalization", SIAM J. Math. Anal., 25:2 (1994), 429-440.

[79] В.П. Спиридонов, "Непрерывная биортогональность эллиптической гипергеометрической функции", Алгебра и анализ (в печати); $\operatorname{arXiv:0801.4137.~}$

[80] H. Rosengren, "Sklyanin invariant integration", Int. Math. Res. Not., 2004, №60, 3207-3232; arXiv: math/0405072.

[81] Е.К. Склянин, "О некоторых алгебраических структурах, связанных с уравнением Янга-Бакстера", Функи. анализ и его прил., 16:4 (1982), 27-34; англ. пер.: E. K. Sklyanin, "Some algebraic structures connected with the Yang-Baxter equation", Funct. Anal. Appl., 16:4 (1982), 263-270.

[82] Е.К. Склянин, "О некоторых алгебраических структурах, связанных с уравнением Янга-Бакстера. Представления квантовой алгебры”, Функи. анализ и его прил., 17:4 (1983), 34-48; англ. пер.: E. K. Sklyanin, "Some algebraic structures connected with the Yang-Baxter equation. Representations of quantum algebras", Funct. Anal. Appl., 17:4 (1983), 273-284.

[83] Yu. I. Manin, "Sixth Painlevé equation, universal elliptic curve, and mirror of $P^{2}$ ", Geometry of differential equations, Amer. Math. Soc. Transl. Ser. 2, 186, Amer. Math. Soc., Providence, RI, 1998, 131-151.

[84] L. D. Faddeev, "Modular double of a quantum group", Conférence Moshé Flato, vol. I (Dijon, 1999), Math. Phys. Stud., 21, Kluwer, Dordrecht, 2000, 149-156.

[85] Э. Т. Уиттекер, Дж. Н. Ватсон, Курс современного анализа, Ч. 2: Трансцендентные функиии, М., 1963; пер. с англ.: Е. T. Whittaker, G. N. Watson, A course of modern analysis, Cambridge Univ. Press., Cambridge, 1915.

[86] H. Rosengren, "Elliptic hypergeometric series on root systems", Adv. Math., 181:2 (2004), 417-447.

[87] R. A. Gustafson, "Multilateral summation theorems for ordinary and basic hypergeometric series in U(n)", SIAM J. Math. Anal., 18:6 (1987), 1576-1596.

[88] C. Krattenthaler, "The major counting of nonintersecting lattice paths and generating functions for tableaux", Mem. Amer. Math. Soc., 115:552 (1995).

[89] Y. Kajihara, M. Noumi, "Multiple elliptic hypergeometric series. An approach from the Cauchy determinant", Indag. Math. (N.S.), 14:3-4 (2003), 395-421. 
[90] H. Rosengren, M. Schlosser, "Summations and transformations for multiple basic and elliptic hypergeometric series by determinant evaluations", Indag. Math. (N.S.), 14:3-4 (2003), 483-513.

[91] E. M. Rains, "Recurrences for elliptic hypergeometric integrals", Rokko Lect. Math., 18 (2005), 183-199; arXiv: math/0504285.

[92] H. Rosengren, M. Schlosser, "Elliptic determinant evaluations and the Macdonald identities for affine root systems", Compos. Math., 142:4 (2006), 937-961.

[93] H. Rosengren, "Sums of triangular numbers from the Frobenius determinant", Adv. Math., 208:2 (2007), 935-961.

[94] H. Rosengren, An Izergin-Korepin-type identity for the 8 VSOS model, with applications to alternating sign matrices, arXiv: 0801.1229.

[95] R. A. Gustafson, "Some $q$-beta and Mellin-Barnes integrals with many parameters associated to the classical groups", SIAM J. Math. Anal., 23:2 (1992), 525-551.

[96] R. A. Gustafson, "Some $q$-beta integrals on $S U(n)$ and $S p(n)$ that generalize the Askey-Wilson and Nassrallah-Rahman integrals", SIAM J. Math. Anal., 25:2 (1994), 441-449.

[97] P. J. Forrester, S. O. Warnaar, "The importance of the Selberg integral", Bull. Amer. Math. Soc. (N.S.), to appear; arXiv: 0710.3981.

[98] I. G. Macdonald, "Constant term identities, orthogonal polynomials, and affine Hecke algebras", Doc. Math., I (1998), 303-317.

[99] G. W. Anderson, "A short proof of Selberg's generalized beta formula", Forum Math., 3:4 (1991), 415-417.

[100] R. A. Gustafson, M. A. Rakha, " $q$-Beta integrals and multivariate basic hypergeometric series associated to root systems of type $A_{m}$ ", Ann. Comb., 4:3-4 (2000), $347-373$.

[101] J. F. van Diejen, V.P. Spiridonov, "Modular hypergeometric residue sums of elliptic Selberg integrals", Lett. Math. Phys., 58:3 (2001), 223-238.

[102] H. Coskun, R. A. Gustafson, "Well-poised Macdonald functions $W_{\lambda}$ and Jackson coefficients $\omega_{\lambda}$ on $B C_{n}$ ", Jack, Hall-Littlewood and Macdonald polynomials, Contemp. Math., 417, Amer. Math. Soc., Providence, RI, 2006, 127-155.

[103] R. Y. Denis, R. A. Gustafson, "An $S U(n) q$-beta integral transformation and multiple hypergeometric series identities", SIAM J. Math. Anal., 23:2 (1992), 552-561.

[104] S. C. Milne, "Multiple $q$-series and $U(n)$ generalizations of Ramanujan's ${ }_{1} \Psi_{1}$ sum", Ramanujan revisited (Urbana-Champaign, IL, 1987), Academic Press, Boston, MA, 1988, 473-524.

[105] A. L. Dixon, "Generalisations of Legendre's formula $K E^{\prime}-(K-E) K^{\prime}=\frac{1}{2} \pi$ ", Proc. London Math. Soc., 2:1 (1905), 206-224.

[106] V.P. Spiridonov, A.S. Zhedanov, "Elliptic grids, rational functions, and the Padé interpolation", Ramanujan J., 13:1-3 (2007), 285-310.

[107] A. Zhedanov, "Padé interpolation table and biorthogonal rational functions", Rokko Lect. Math., 18 (2005), 323-363.

[108] T. H. Koornwinder, "Askey-Wilson polynomials for root systems of type BC", Hypergeometric functions on domains of positivity, Jack polynomials, and applications (Tampa, FL, 1991), Contemp. Math., 138, Amer. Math. Soc., Providence, RI, 1992, 189-204.

[109] A. Okounkov, "BC-type interpolation Macdonald polynomials and binomial formula for Koornwinder polynomials", Transform. Groups, 3:2 (1998), 181-207.

[110] G. Felder, A. Varchenko, "Hypergeometric theta functions and elliptic Macdonald polynomials", Int. Math. Res. Not., 2004, № 21, 1037-1055. 
[111] H. Sakai, "Rational surfaces associated with affine root systems and geometry of the Painlevé equations", Comm. Math. Phys., 220:1 (2001), 165-229.

[112] K. Kajiwara, T. Masuda, M. Noumi, Y. Ohta, Y. Yamada, " ${ }_{10} E_{9}$ solution to the elliptic Painlevé equation", J. Phys. A, 36:17 (2003), L263-L272.

[113] M. Ito, "Askey-Wilson type integrals associated with root systems", Ramanujan J., 12:1 (2006), 131-151.

[114] M.E.H. Ismail, M. Rahman, "The associated Askey-Wilson polynomials", Trans. Amer. Math. Soc., 328:1 (1991), 201-237.

[115] V.P. Spiridonov, "A multiparameter summation formula for Riemann theta functions", Jack, Hall-Littlewood and Macdonald polynomials, Contemp. Math., 417, Amer. Math. Soc., Providence, RI, 2006, 345-353.

[116] H. Konno, "The vertex-face correspondence and the elliptic $6 j$-symbols", Lett. Math. Phys., 72:3 (2005), 243-258; arXiv: math/0503725.

[117] Н.И. Ахиезер, Элементы теории эллиптических функиий, Наука, М., 1970; англ. пер.: N. I. Akhiezer, Elements of the theory of elliptic functions, Transl. Math. Monogr., 79, Amer. Math. Soc., Providence, RI, 1990.

[118] M. Eichler, D. Zagier, The theory of Jacobi forms, Progr. Math., 55, Birkhäuser, Boston, MA, 1985.

В. П. Спиридонов (V.P. Spiridonov)

Лаборатория теоретической физики ОИЯИ, Дубна

E-mail: spiridon@theor.jinr.ru
Поступила в редакцию

09.04 .2008 